\title{
Genomically Mined Acoustic Reporter Genes Enable In Vivo Monitoring of Tumors and Tumor-Homing Bacteria
}

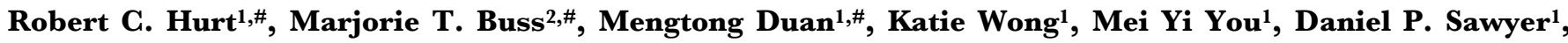 \\ Margaret B. Swift ${ }^{2}$, Przemysław Dutka ${ }^{1,2}$, David R. Mittelstein ${ }^{3}$, Zhiyang Jin ${ }^{3}$, Mohamad H. Abedi', Arash \\ Farhadi', Ramya Deshpande², Mikhail G. Shapiro',** \\ ${ }^{1}$ Division of Biology and Biological Engineering, California Institute of Technology, Pasadena, CA, USA \\ ${ }^{2}$ Division of Chemistry and Chemical Engineering, California Institute of Technology, Pasadena, CA, USA \\ ${ }^{3}$ Division of Engineering and Applied Sciences, California Institute of Technology, Pasadena, CA, USA \\ \#Equal contribution \\ *Correspondence should be addressed to M.G.S. (mikhail@caltech.edu)
}

\begin{abstract}
A major outstanding challenge in the fields of biological research, synthetic biology and cell-based medicine is the difficulty of visualizing the function of natural and engineered cells noninvasively inside opaque organisms. Ultrasound imaging has the potential to address this challenge as a widely available technique with a tissue penetration of several centimeters and spatial resolution below $100 \mu \mathrm{m}$. Recently, the first genetically encoded acoustic reporters were developed based on bacterial gas vesicles to link ultrasound signals to molecular and cellular function. However, the properties of these first-generation acoustic reporter genes (ARGs) resulted in limited sensitivity and specificity for imaging in the in vivo context. Here, we describe second-generation ARGs with greatly improved acoustic properties and expression characteristics, identified through a phylogenetic screen of candidate gene clusters from diverse bacteria and archaea. The resulting constructs offer major qualitative and quantitative improvements, including much stronger ultrasound contrast, the ability to produce nonlinear signals distinguishable from background tissue, and stable long-term expression. We demonstrate the utility of these next-generation ARGs by imaging in situ gene expression in mouse models of breast cancer and tumor-homing probiotic bacteria, revealing the unique spatial distributions of tumor growth and colonization by therapeutic cells noninvasively in living subjects.
\end{abstract}

\section{INTRODUGTION}

Basic biological research, in vivo synthetic biology and the development of cell-based medicine require methods to visualize the functions of specific cells deep inside intact organisms. Within this context, widely used optical techniques based on fluorescent and luminescent proteins have limited utility due to the scattering and absorption of light by tissue. ${ }^{1}$ In contrast, ultrasound is a widely used technique for deeptissue imaging, providing sub-100 $\mu$ m spatial resolution and penetrating several $\mathrm{cm}$ into tissue. ${ }^{2}$ The relative simplicity and low cost of ultrasound make it widely accessible for both research and clinical medicine. Recently, the first genetically encodable reporters for ultrasound were introduced based on gas vesicles $(\mathrm{GVs})$ : $^{3}$ air-filled protein nanostructures encoded by clusters of $8-20+$ genes, which evolved as flotation devices in a wide range of mostly aquatic bacteria and archaea. ${ }^{4,5}$ The low density and high compressibility of their air-filled interiors compared to surrounding tissues allow GVs to scatter sound waves and thereby produce ultrasound contrast when heterologously expressed as acoustic reporter genes (ARGs) in genetically engineered bacteria ${ }^{6}$ or mammalian cells. ${ }^{7}$

Despite the promise of first-generation ARGs, several limitations prevent their widespread use for monitoring bacterial or mammalian cells in vivo. First-generation bacterial $\mathrm{ARGs}^{6}$ could not scatter ultrasound nonlinearly (making them difficult to distinguish from background tissues), expressed poorly at $37^{\circ} \mathrm{C}$, and were too metabolically burdensome for in situ expression in vivo. Likewise, first-generation mammalian $\mathrm{ARGs}^{7}$ also produced only linear contrast, and cell-to-cell variability in their expression and burden meant that they could only be imaged robustly with ultrasound in clonally selected cell lines. In both cases, the lack of nonlinear signal had to be circumvented by destructive ultrasound pulse sequences, which destroyed the $\mathrm{GVs}$ and limited dynamic imaging. ${ }^{8}$

To overcome these limitations, we sought nextgeneration ARGs that, when expressed heterologously in either probiotic bacterial strains or mammalian cancer cell lines, could produce GVs with strong nonlinear ultrasound contrast and enable strong, sustained expression under physiological conditions. These qualities would enable longterm noninvasive imaging of gene expression in a broad range of in vivo applications. We hypothesized that a genomic mining approach - previously applied to improving fluorescent proteins, optogenetic receptors and other biotechnology tools ${ }^{9}$ 13 - would yield ARGs with improved properties, which could be further optimized through genetic engineering. By cloning and screening 15 distinct polycistronic operons from a diverse set of GV-expressing species representing a broad phylogeny, we identified two GV gene clusters - from Serratia sp. 39006 and Anabaena flos-aquae - that produce vastly more linear and nonlinear acoustic contrast than previously tested clusters when expressed in bacteria or mammalian cells, respectively. The ARG adapted from Serratia sp. 39006 (bARG Ser $_{\text {), when }}$ expressed in the widely used probiotic bacterium $E$. coli Nissle 1917 (EcN), enabled noninvasive ultrasound imaging of these probiotic agents colonizing tumors, providing direct visualization of the microscale in vivo distribution of this rapidly emerging class of anti-cancer therapy. The mammalian ARG adapted from $A$. flos-aquae ( $\mathrm{mARG}_{\text {Ana }}$ ), when expressed in the human breast cancer cell line MDA-MB-231, enabled the noninvasive, in situ microscale imaging and long-term 
bioRxiv preprint doi: https://doi.org/10.1101/2021.04.26.441537; this version posted October 29, 2021. The copyright holder for this preprint (which was not certified by peer review) is the author/funder. All rights reserved. No reuse allowed without permission.

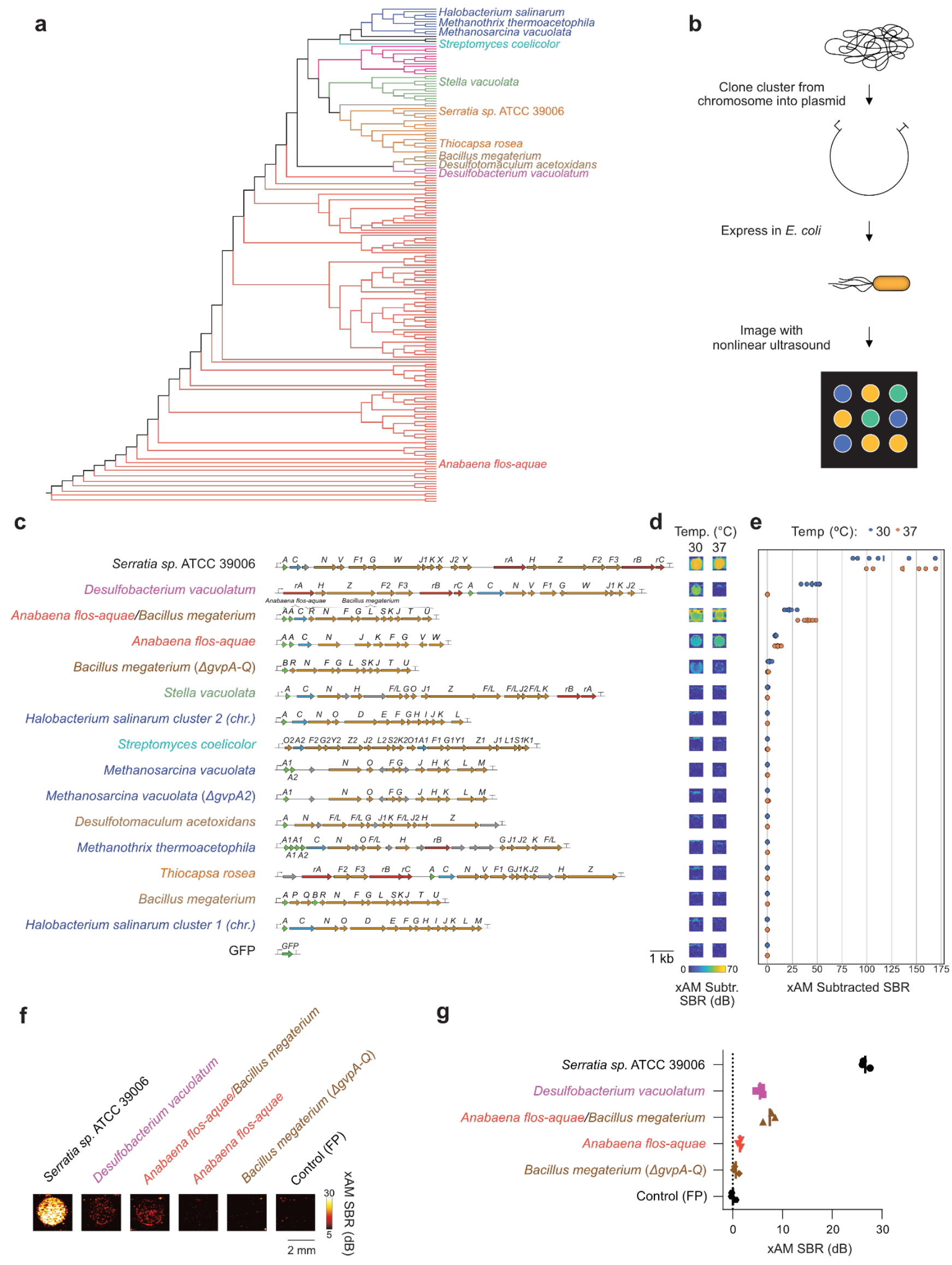


Figure 1 | Genomic mining of gas vesicle gene clusters reveals homologs with nonlinear ultrasound contrast in $E$. coli. (a) $16 \mathrm{~S}$ phylogenic tree of known GV-producing organisms, with the species from which GV genes were cloned and tested in this study indicated by name. See Fig. S1 for the fully annotated phylogenic tree. B. megaterium and S. coelicolor were not reported to produce GVs, but we tested their GV gene clusters here based on previous experiments in E. coli ${ }^{3}$ and to broadly sample the phylogenetic space. (b) Workflow for testing GV clusters. Select GV gene clusters were expressed in BL21(DE3) E. coli by growing patches of cells on plates containing the inducer IPTG, and the patches were then imaged with nonlinear ultrasound (xAM). (c-e) Diagrams of the GV gene clusters tested in E. coli (c), pre-minuspost-collapse XAM images of representative patches (d), and quantification of the pre-minus-post-collapse xAM signal-to-background ratio (SBR) of the patches ( $\mathrm{n}=6$ biological replicates) (e). (f-g) Representative $\mathrm{xAM}$ images (f) and quantification of the $\mathrm{xAM}$ SBR ( $\mathrm{n}=3$ biological replicates, each with 2 technical replicates) (g) for the top $5 \mathrm{GV}$-producing clusters expressed in E. coli at $30^{\circ} \mathrm{C}$ on solid media and normalized to $5 \times 10^{9}$ cells $/ \mathrm{mL}$ in agarose phantoms, imaged at $1.74 \mathrm{MPa}$. See Fig. S7a-b for the ultrasound signal at varying acoustic pressures and Fig. S7c for the corresponding BURST data.

monitoring of heterologous gene expression activity in developing orthotopic tumors.

\section{RESULTS}

Genomic mining of gas vesicle gene clusters reveals homologs with improved ultrasound performance in

E. coli. $\mathrm{GVs}$ are encoded by polycistronic gene clusters comprising one or more copies of the primary structural gene gvpA and 7 to $20+$ other genes encoding minor constituents, assembly factors or reinforcing proteins, which together help assemble the GVs' protein shell. ${ }^{5}$ Hundreds of organisms have GV genes in their genomes, but only a small subset have been shown to produce GVs. Given the labor involved in cloning and testing polycistronic clusters, we limited our phylogenetic search to organisms with confirmed GV production. From a list of 253 unique $\mathrm{GV}$-producing species and 288 unique strains in the literature (Table S1), 117 have had their GV operons sequenced. We created a phylogenetic tree based on the 16S rDNA sequences of these organisms (Fig. 1a and S1) and used it to select 11 representative species, broadly sampling phylogenetic space, cluster architecture and organismal characteristics (i.e., halophilic, thermophilic and mesophilic). We obtained each species from culture repositories, amplified $\mathrm{GV}$ operons from their genomes, and cloned them into a bacterial expression vector.

We then expressed each operon in confluent $E$. coli patches at several temperatures and inducer concentrations (Fig. 1b), comparing them to two bacterial ARG constructs previously shown to work in E. coli - bARG1 (Anabaena flosaquae/Bacillus megaterium hybrid) and Bacillus megaterium $4 g v p A-$ $Q$ as well as the full Bacillus megaterium gene cluster (Fig. 1c-g, S2a-e, S3a-c, S4-6). We scanned these patches using a homebuilt robot-translated ultrasound imaging apparatus, applying a nonlinear ultrasound pulse sequence based on the transmission of cross-propagating waves for amplitude modulation (xAM). ${ }^{14}$ This pulse sequence enhances signals specific to nonlinear contrast agents such as GVs while reducing background tissue scattering. Importantly, unlike other pulse sequences that rely on the irreversible collapse of $\mathrm{GV}$ s to obtain $\mathrm{GV}$-specific contrast, $\mathrm{xAM}$ is nondestructive. In addition, we examined the optical opacity of the patches, which can be increased by sufficient levels of GV expression.

Of the 15 gene clusters tested, only 3 showed significant $\mathrm{xAM}$ signal when expressed at $37^{\circ} \mathrm{C}$, and 5 showed significant $\mathrm{xAM}$ signal at $30^{\circ} \mathrm{C}$ (Fig. 1c-e). In addition, only 3 clusters produced opaque patches at $30^{\circ} \mathrm{C}$, all of which also produced significant $x A M$ signal at this temperature $($ Fig. S2c and S5a-b). Even though all operons tested are from organisms reported to produce GVs in their native hosts, only the Anabaena flos-aquae, Bacillus megaterium $\operatorname{sop} A-Q$ bARG1, Desulfobacterium vacuolatum, and Serratia sp. 39006 (Serratia) clusters produced GVs heterologously in E. coli. Several other operons produced a small amount of ultrasound contrast under certain conditions, which did not arise from GV expression but reflected a rough patch morphology likely due to cellular toxicity (Fig. S3d). The failure of most tested gene clusters to produce $\mathrm{GVs}$ in $E$. coli is not surprising given the complexity of polycistronic heterologous expression, which requires each component to fold and function properly in a new host with a potentially different cytoplasmic environment, growth temperature and turgor pressure. ${ }^{15,16}$ In addition, it is possible that some genes included in the clusters act as cisregulators, , $^{5,15,17-19}$ limiting expression absent a specific trans input, or that some additional genes are required beyond the annotated operons.

In patch format, the strongest acoustic performance was observed with the genes from Serratia, bARG1, A. flos-aquae, B. megateriaum, and D. vacuolatum. Because patch experiments do not control for the density of cells in each sample, we further compared the performance of these clusters in resuspended samples. Each operon was expressed on solid media at $30^{\circ} \mathrm{C}-$ a temperature at which all five operons produced $\mathrm{GVS}_{\mathrm{s}}$ - then scraped, resuspended, and normalized for cell concentration. These samples were imaged in hydrogels using xAM (Fig. 1fg and S7a-b) and a more sensitive but destructive imaging method called BURST ${ }^{20}$ (Fig. S7c), and examined optically with phase-contrast microscopy (PCM), which reveals the presence of $\mathrm{GVs}$ due to the refractive index difference between GVs and water ${ }^{21,22}$ (Fig. S7d). Three of the clusters produced xAM signals, and all clusters produced BURST signals significantly stronger than the negative control. All clusters except $A$. flos-aquae exhibited sufficient GV expression to be visible by PCM.

Cells expressing the Serratia cluster produced the strongest ultrasound signals, $19.2 \mathrm{~dB}$ above the next brightest cluster, bARG1, under xAM imaging at an applied acoustic pressure of 1.74 $\mathrm{MPa}$ - an 83-fold gain in signal intensity (Fig. 1f). Additionally, PCM images (Fig. S7d) showed that cells expressing the Serratia cluster had the highest levels of GV expression, as also seen in whole-cell transmission electron microscopy (TEM) (Fig. S7e). Based on the large improvement in ultrasound contrast provided by the Serratia GV operon relative to the other gene clusters, we selected this operon for further optimization as a second-generation bacterial ARG. 
a
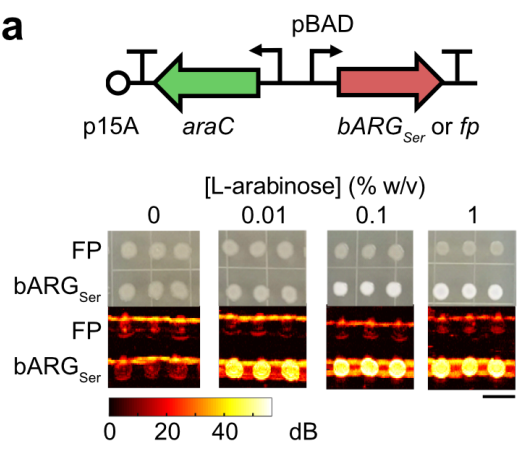

d

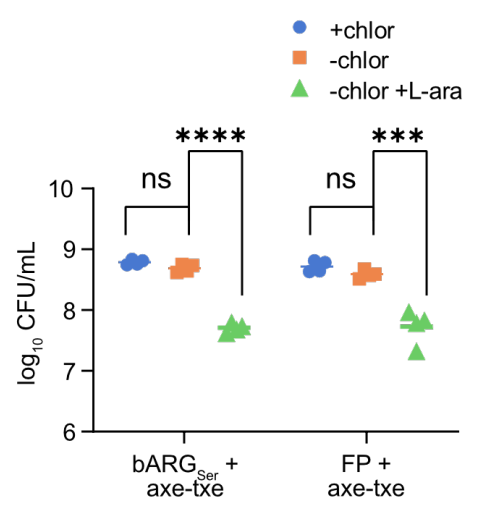

g

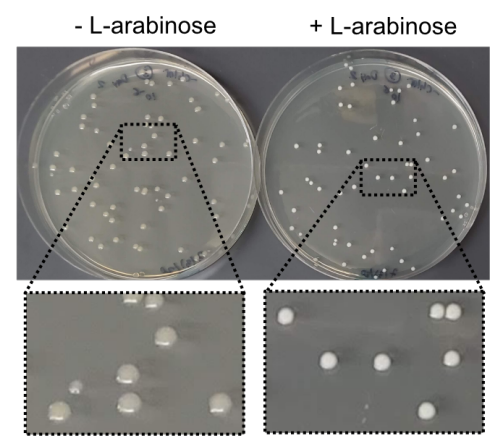

b

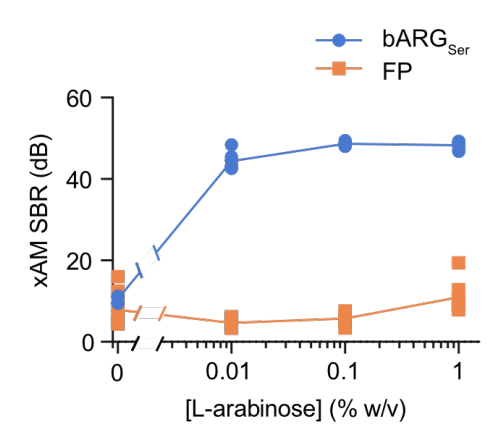

e
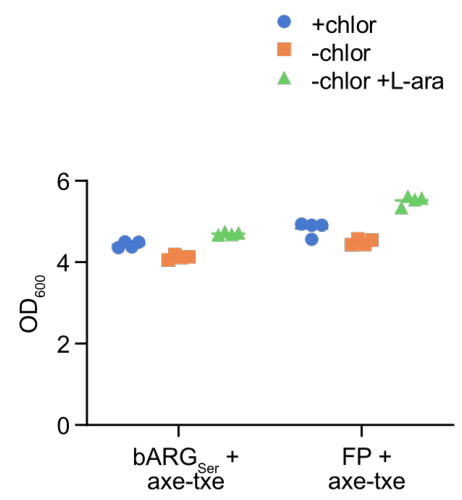

h

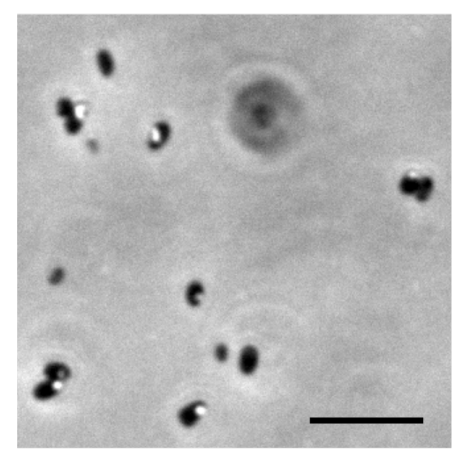

C
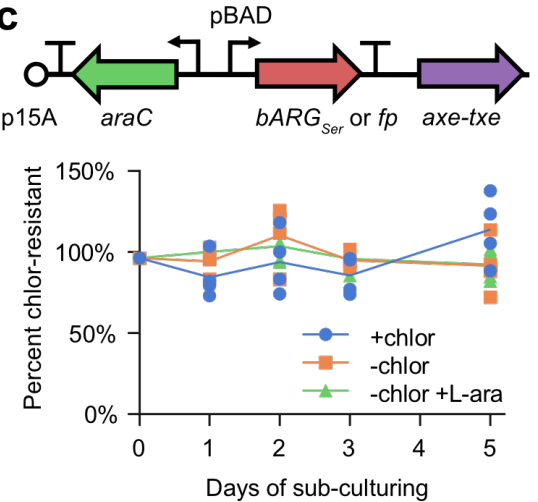

f

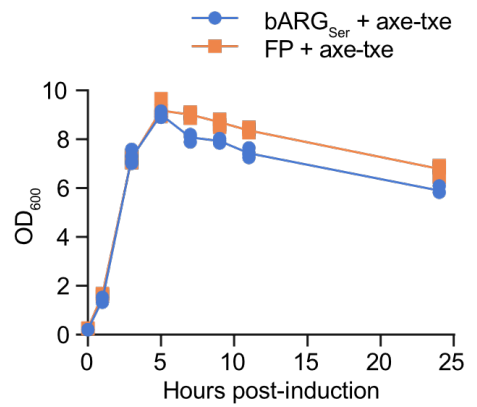

i

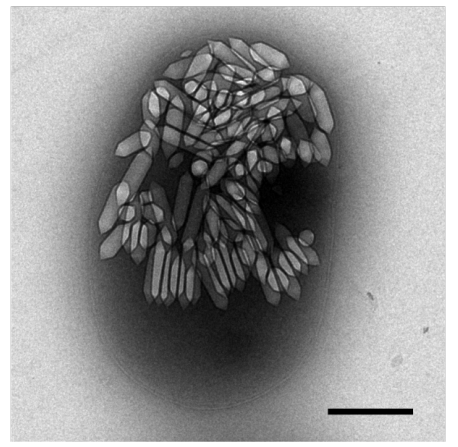

Figure 2 | Genetic engineering and expression of bARG the arabinose-inducible construct used to express bARG $\mathrm{Ser}_{\text {in }} \mathrm{EcN}$ (top), and optical and xAM images of bARG $\mathrm{Ger}_{\text {-expressing or FP- }}$ expressing patches of $\mathrm{EcN}$ on solid media with varying L-arabinose concentrations at $37 \circ \mathrm{C}$ (bottom). The scale bar is $1 \mathrm{~cm}$. See Fig. S9 for the corresponding results with IPTG-inducible and aTc-inducible constructs. (b) Quantification of the xAM SBR of all patches from the experiment in (a) versus the L-arabinose concentration ( $n=8$ biological replicates). (c) Diagram of the construct from (a) with the toxinantitoxin stability cassette Axe-Txe ${ }^{29}$ added to enable plasmid maintenance in the absence of antibiotics (top), and verification of plasmid maintenance in vitro (bottom). The percentage of chloramphenicol-resistant colonies was measured during daily sub-culturing into LB media with $25 \mu \mathrm{g} / \mathrm{mL}$ chloramphenicol (+chlor), without chloramphenicol (-chlor), or without chloramphenicol and with $0.1 \%$ (w/v) L-arabinose (-chlor $+\mathrm{L}$-ara) using pBAD-bARG $\mathrm{Ser}_{-A x e T x e} \mathrm{EcN}$ ( $\mathrm{n}=4$ biological replicates). The percentage of chloramphenicol-resistant colonies was calculated by dividing the number of colonies on plates with chloramphenicol by the number of colonies on plates without chloramphenicol. (d-e) $\log 10$ of the colony forming units (CFUs) on chloramphenicol plates per mL of culture (d) and optical density at $600 \mathrm{~nm}$ (e) of pBADbARGser-AxeTxe EcN and pBAD-FP-AxeTxe EcN cultures 24 hours after sub-culturing into LB media with the same conditions as in (c). Asterisks indicate statistical significance by unpaired t tests $(* * * *=p<0.0001$, *** $=p<0.001$, ns $=$ no significance); $\mathrm{n}=4$ biological replicates. (f) $\mathrm{OD}_{600}$ versus time after inducing pBAD-bARG $\mathrm{Ser}_{-A x e T x e}\left(\mathrm{bARG} \mathrm{G}_{\text {Ser }}+\right.$ axe-txe) and pBAD-FP-AxeTxe $(\mathrm{FP}+$ axe-txe) EcN strains with $0.1 \%(\mathrm{w} / \mathrm{v})$ L-arabinose in liquid culture at $37 \mathrm{oC}(\mathrm{n}=4$ biological replicates). Between 5 and 24 hours post-induction, when the $\mathrm{OD}_{600}$ of all cultures decreased, the $\mathrm{OD}_{600}$ of FP-expressing cultures was slightly higher than that of the bARGser-expressing cultures, likely due to expression of red fluorescent protein which is known to absorb light at $600 \mathrm{~nm} .{ }^{42}$ (g) Representative image of colonies from the experiment in (c) on chloramphenicol plates with (right) and without (left) $0.1 \%$ (w/v) L-arabinose. The opacity of the colonies on plates with L-arabinose indicates bARGser expression and was used to screen for mutants deficient in bARGser expression (see Fig. S1 1). (h-i) Representative phase contrast microscopy (h) and transmission electron microscopy (i) images of pBAD-bARG on plates with $0.1 \%(\mathrm{w} / \mathrm{v})$ L-arabinose at $37 \circ \mathrm{C}$. Scale bars are $10 \mu \mathrm{m}(\mathrm{h})$ and $500 \mathrm{~nm}(\mathrm{i})$. 
Because overexpression of any protein imposes a finite metabolic demand on the host cell, ${ }^{23-25}$ we reasoned that deletion of non-essential genes could improve GV expression from the Serratia cluster, and therefore the xAM signal. Previous work showed that deletions of $g v p C, g v p W, g v p X$, gvp $Y$, gopH, or gopZ preserve GV formation in the native organism. ${ }^{15}$ We tested these deletions, as well as the deletion of an unannotated hypothetical protein (Ser39006_001280) encoded between the gvpC and grp $\mathcal{N}$ coding sequences (Fig. S8a). When expressed in $E$. coli, deletions of $g v p C, g v p H, g v p W$, gvp $Y$, or gvpZ reduced or eliminated XAM signal (Fig. S8b-c) and patch opacity (Fig. S8d). Deletion of gop $X$ increased xAM signal but decreased opacity, and deletion of Ser39006_001280 increased both xAM signal and opacity. Based on these results, we chose to use the Serratia $\Delta$ Ser39006_001280 operon for subsequent in vitro and in vivo experiments. We call this genetic construct $\mathrm{bARG}_{\mathrm{Ser}}-\mathrm{a}$ bacterial acoustic reporter gene derived from Serratia.

bARG $G_{\text {Ser }}$ shows robust expression, contrast, and stability in $\boldsymbol{E}$. coli Nissle. We transferred $\mathrm{bARG}_{\mathrm{Ser}}$ into $\mathrm{EcN}$, a strain of $E$. coli that is widely used in in vivo biotechnology applications due to its ability to persist in the gastrointestinal tract and colonize tumors. ${ }^{26-28}$ Recently, EcN has been intensely investigated as the chassis for anti-tumor therapy delivery. ${ }^{29-32}$ We first tested different inducible promoter architectures in $\mathrm{EcN}$ on solid media at $37^{\circ} \mathrm{C}$, examining xAM ultrasound contrast and patch opacity as a function of inducer concentration. We found that the Larabinose-inducible $\mathrm{pBAD}$ promoter provided the most robust control over GV expression without obvious burden (Fig. 2ab, Fig. S9). Based on these results, we selected the pBAD$\mathrm{bARG}_{\text {Ser }} \mathrm{EcN}$ strain for subsequent experiments.

To ensure that the $\mathrm{pBAD}^{\mathrm{b} A R \mathrm{G}_{\text {Ser }}}$ plasmid is maintained in the absence of antibiotic selection, as required in certain in vivo applications, we added the toxin-antitoxin stability cassette Axe-Txe. ${ }^{33}$ This enabled the pBAD-bARG ${ }_{\text {Ser- }}$ AxeTxe plasmid to be maintained in $\mathrm{EcN}$ for up to 5 days of daily sub-culturing in liquid media without antibiotics, both with and without induction of ARG expression $(\mathbf{F i g} . \mathbf{2 c})$.

The expression of most heterologous genes, including widely used reporter genes such as fluorescent proteins, results in some degree of metabolic burden on engineered cells. ${ }^{24,25,34}$ Consistent with this expectation, the induction of pBADbARG $_{\text {Ser }} \mathrm{EcN}_{\mathrm{N}}$ resulted in reduced colony formation to an extent similar to the expression of a fluorescent protein (FP) (Fig. 2d and S10a), even as the culture density measured by $\mathrm{OD}_{600}$ remained relatively unchanged (Fig. 2 e and $\mathbf{S 1 0 b}$ ). When the $\mathrm{OD}_{600}$ was measured after inducing cultures with $\mathrm{L}$ arabinose, the growth curves of bARG $\mathrm{SSe}_{\mathrm{Se}}$-expressing and FPexpressing $\mathrm{EcN}$ were indistinguishable during the growth phase ( 0 to 5 hours), indicating that the two strains have similar growth rates (Fig. 2f). Collectively, these results suggest that overexpression of $\mathrm{bARG}_{\mathrm{Ser}}$ using the $\mathrm{pBAD}$ expression system in $\mathrm{EcN}$ is not significantly more burdensome than that of FPs, which are widely accepted as relatively non-perturbative indicators of cellular function.
To further examine the genetic stability of bARG Ser constructs, we plated cells from daily sub-cultures onto agar with $0.1 \%(\mathrm{w} / \mathrm{v})$ L-arabinose and examined colony opacity (Fig. 2g) as a measure of retained GV expression. Of a total of 3824 colonies, nearly all were opaque (Fig. 2 g), with GV expression confirmed by PCM and TEM (Fig. 2h-i). Only 11 colonies $(<0.3 \%$ after $\sim 35$ cell generations) exhibited a reduced opacity (Fig. S11a), representing a mutated phenotype confirmed by growing these cells on fresh media (Fig. S11b). PGM revealed that these rare mutants still produced GVs, but at lower levels than non-mutants. These results indicate that mutational inactivation of $\mathrm{GV}$ production is not a major issue for pBAD-bARG ${ }_{S e r}-A x e T x e ~ E c N$ under typical conditions.

After establishing construct stability, we characterized the acoustic properties of bARG $\mathrm{Ser}_{\mathrm{Se}}$-expressing EcN. For cells induced with $0.1 \% \mathrm{~L}$-arabinose for 24 hours and suspended at $10^{9}$ cells $/ \mathrm{mL}$ in agarose phantoms, an $\mathrm{xAM}$ signal was detected at acoustic pressures above $0.72 \mathrm{MPa}$, rising with increasing pressure up to the tested maximum of $1.74 \mathrm{MPa}$ (Fig. 3a). To characterize the physical stability of GVs in these cells during ultrasound exposure, we measured the xAM signal over time at a series of increasing acoustic pressures (Fig. 3b). The xAM signal was steady at pressures up to $0.96 \mathrm{MPa}$, at which point we observed a slow decrease, indicating that some of the GVs slowly collapsed despite sustained high xAM signals. We also imaged the cells with parabolic pulses, which can transmit higher pressures than XAM, and thus can be helpful in vivo to compensate for attenuation at tissue interfaces. When imaged with parabolic B-mode at varying acoustic pressures, the GVs started to collapse slowly at $1.02 \mathrm{MPa}$ and more rapidly at $1.33 \mathrm{MPa}$ and above (Fig. 3c). Based on these results, an acoustic pressure of $1.29 \mathrm{MPa}$ was selected for xAM and 1.02 MPa for parabolic AM (pAM) imaging in subsequent experiments to obtain the strongest signals while minimizing GV collapse.

Next, to characterize the dynamics and inducibility of bARG $_{\text {Ser }}$ in $\mathrm{EcN}$ and determine the ultrasound detection limit of bARG $\mathrm{Ser}_{\mathrm{Ser}}$-expressing $\mathrm{EcN}$, we measured xAM signal as a function of induction time, inducer concentration, and cell concentration. At a density of $10^{9} \mathrm{cells} / \mathrm{mL}$, xAM signal could first be observed 7 hours after induction with $0.1 \% \mathrm{~L}$-arabinose and leveled off by 9 hours post-induction (Fig. 3d-e). Keeping the induction time constant at 24 hours while varying the Larabinose concentration, GV expression was detected with as little as $0.001 \%$ L-arabinose, and the highest ultrasound signal was observed for $0.1-1 \%$ L-arabinose (Fig. 3f-g). When cells induced for 24 hours with $0.1 \%$ L-arabinose were diluted, they were detectable by ultrasound down to $10^{7}$ cells $/ \mathrm{mL}$ (Fig. 3hi). Critically, this detection was achieved non-destructively with nonlinear imaging, compared to previous bacterial ARGs, which required a destructive linear imaging approach. ${ }^{6}$ The bARG $_{\text {Ser }} \mathrm{xAM}$ signal was proportional to the cell concentration between $10^{7}$ cells $/ \mathrm{mL}$ and $2 \times 10^{9}$ cells $/ \mathrm{mL}$ (Fig. 3h-i). We also imaged the cells using BURST imaging, which provides greater sensitivity at the cost of collapsing the GVs. ${ }^{20}$ BURST enabled bARG Ser -expressing $\mathrm{EcN}$ to be detected as early as 3 hours post-induction (Fig. S12a-b), with 

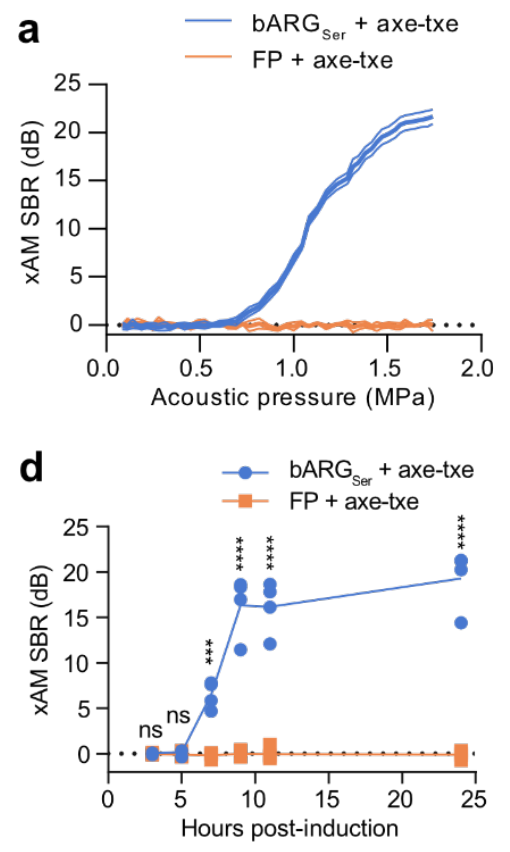

e

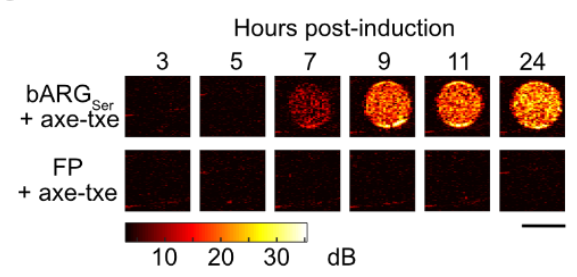

b

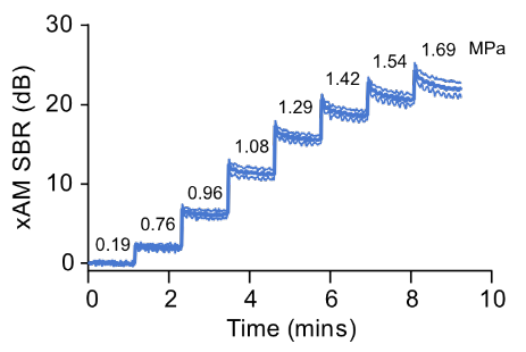

f

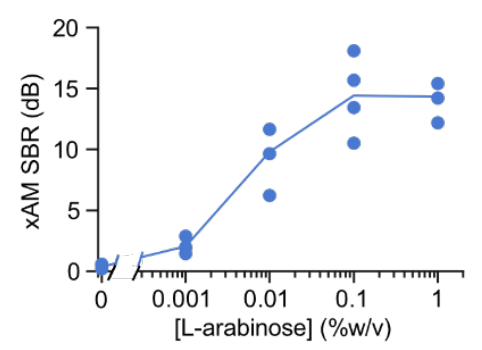

g

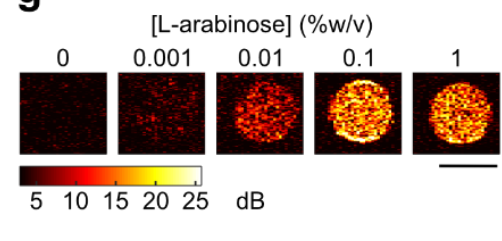

C

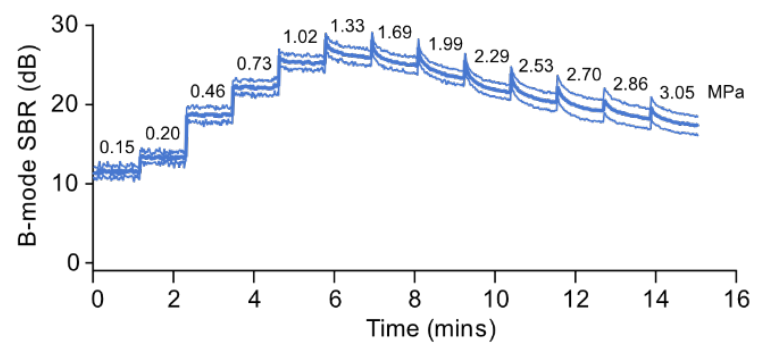

h

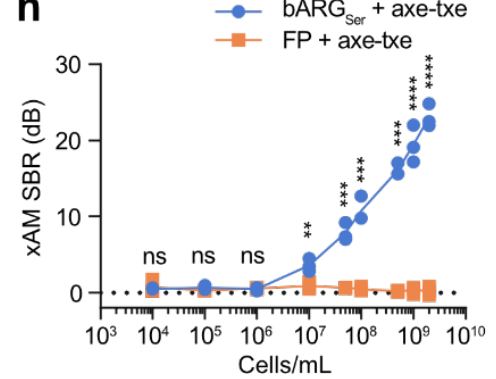

i

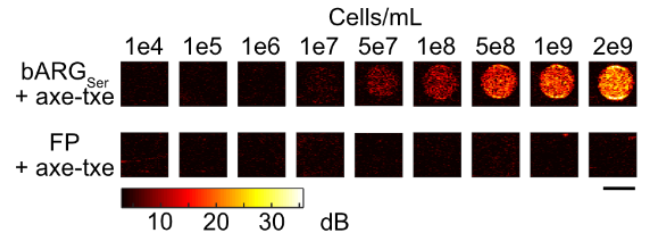

Figure 3 | Acoustic characterization of $\mathbf{b A R G}_{\mathrm{Ser}}$-expressing EcN in vitro. (a) xAM SBR as a function of transmitted acoustic

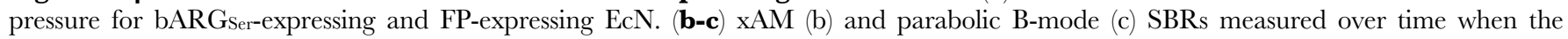
transmitted acoustic pressure was increased approximately every $70 \mathrm{sec}$ as indicated by the numbers above the curve for bARGser-expressing EcN. Ultrasound was applied at a pulse repetition rate of $86.8 \mathrm{~Hz}$. For (a-c), pBAD-bARGser-AxeTxe EcN were induced with 0.1\% (w/v) Larabinose for 24 hours at $37^{\circ} \mathrm{C}$ in liquid culture, and were then normalized to $10^{9}$ cells $/ \mathrm{mL}$ in agarose phantoms for ultrasound imaging. Bold lines represent the mean and thin lines represent \pm standard deviation; $n=3$ biological replicates, each with 2 technical replicates. (d-e) $x A M$ ultrasound SBR (d) and corresponding representative ultrasound images (e) at several time points after inducing pBAD-bARGser-AxeTxe (bARG $_{\text {Ser }}+$ axe-txe) and pBAD-FP-AxeTxe $(\mathrm{FP}+$ axe-txe) EcN strains with $0.1 \%(\mathrm{w} / \mathrm{v})$ L-arabinose in liquid culture at $37 \circ \mathrm{C}$. $(\mathbf{f}-\mathbf{g}) \mathrm{xAM}$ ultrasound SBR (f) and corresponding representative ultrasound images (g) using varying L-arabinose concentrations to induce pBADbARGser-AxeTxe EcN in liquid culture at $37{ }^{\circ} \mathrm{C}$ for 24 hours. (h-i) xAM ultrasound SBR (h) and corresponding representative ultrasound images (i) of varying concentrations of pBAD-bARGser-AxeTxe or pBAD-FP-AxeTxe EcN cells induced for 24 hours at $37 \mathrm{C}^{\circ}$ with $0.1 \%$ (w/v) L-arabinose in liquid culture. For (e, g, i) scale bars are $2 \mathrm{~mm}$. For (d-g), cells were normalized to $10^{9}$ cells $/ \mathrm{mL}$ in agarose phantoms for ultrasound imaging. For (d, f, h), each point is a biological replicate ( $n=4$ for $d$ and $f ; n=3$ for $h$ ) that is the average of at least 2 technical replicates. Asterisks represent statistical significance by unpaired t tests $(* * * *=p<0.0001, * * *=p<0.001, * *=p<0.01$, ns $=$ no significance).

as little as $0.001 \%$ L-arabinose (Fig. S12c-d), and at density as low as $10^{5}$ cells $/ \mathrm{mL}$ (Fig. S12e-f). Taken together, our in vitro experiments indicated that the reporter gene construct

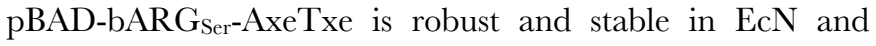
enables gene expression in these cells to be imaged with high contrast and sensitivity.

$b_{A R} G_{\text {Ser }}$ enables in situ imaging of tumor-colonizing bacteria. Tumor-homing bacteria are a major emerging class of cancer therapy, taking advantage of the ability of cells such as $\mathrm{EcN}$ to infiltrate tumors and proliferate in their immunosuppressed microenvironment. Major synthetic biology efforts have been undertaken to turn tumor-homing EcN cells into effective therapies for solid tumors. ${ }^{29-32}$ However, despite the promise of this technology and the importance of appropriate microscale biodistribution of the bacteria inside tumors, no effective methods currently exist to visualize this biodistribution in situ in living animals.

To test the ability of $\mathrm{bARG}_{\text {Ser }}$ to overcome this limitation, we formed subcutaneous MC26 tumors in mice and, when the tumors reached a substantial size, intravenously injected EcN cells containing the pBAD-bARG $G_{\text {Ser-AxeTxe }}$ plasmid, giving the bacteria 3 days to home to and colonize the tumors. We then induced GV expression and imaged the tumors with ultrasound $($ Fig. 4a). In all tumors colonized by

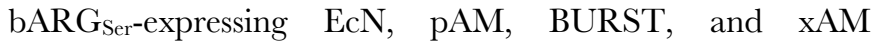
ultrasound signals were observed 1 day after induction with Larabinose (Fig. 4b and S13a). The signals were localized to the core of the tumor and concentrated at the interface between live and necrotic tissue, where the EcN primarily 
bioRxiv preprint doi: https://doi.org/10.1101/2021.04.26.441537; this version posted October 29, 2021. The copyright holder for this preprint (which was not certified by peer review) is the author/funder. All rights reserved. No reuse allowed without permission.

a

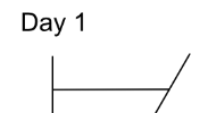

Day 14

Day 17

Day 18

Day 19

Day 20
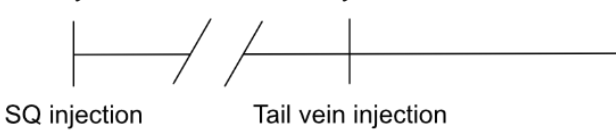

MC26 cells

Tail vein injection

E. coli Nissle

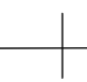

IP injection

L-arabinose

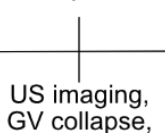

GV collapse,
IP injection
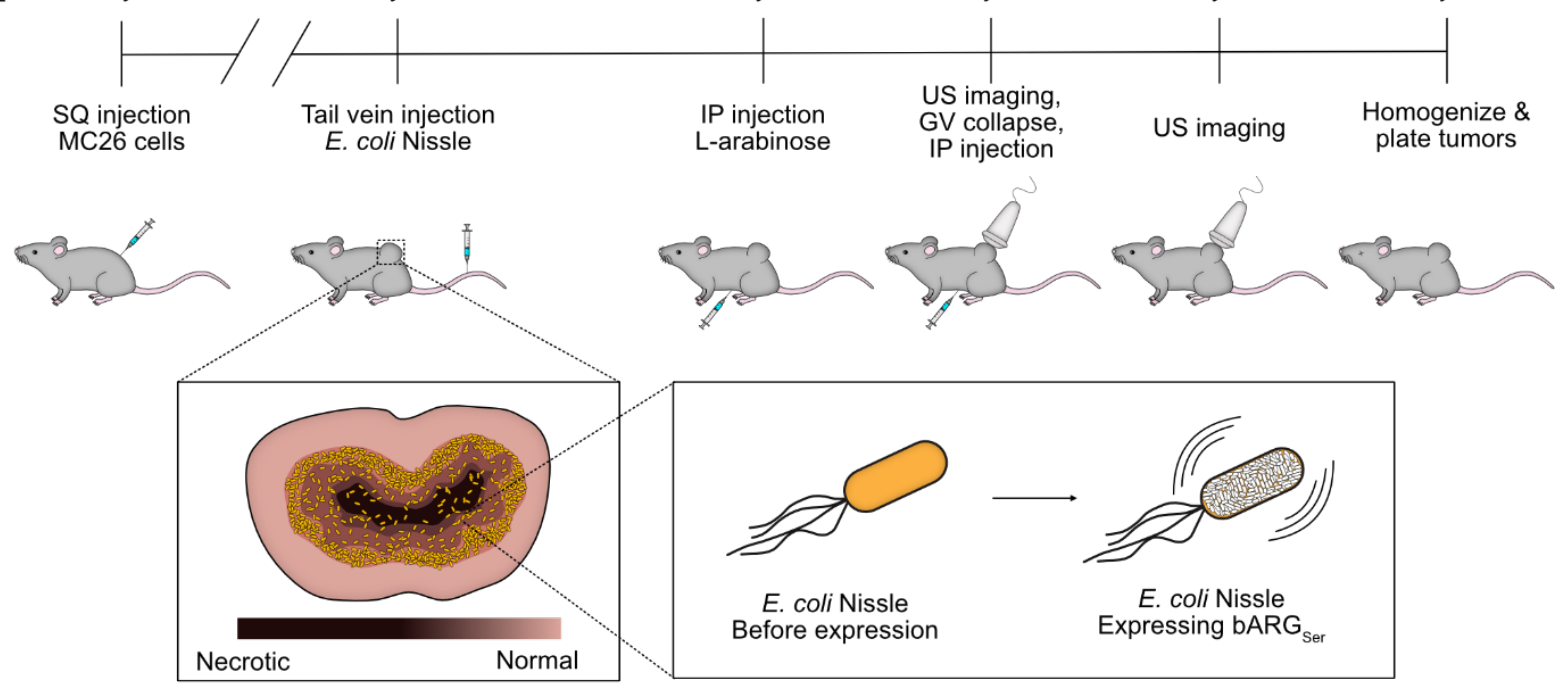

E. coli Nissle

Before expression

E. coli Nissle

Expressing bARG ${ }_{\text {Ser }}$

b
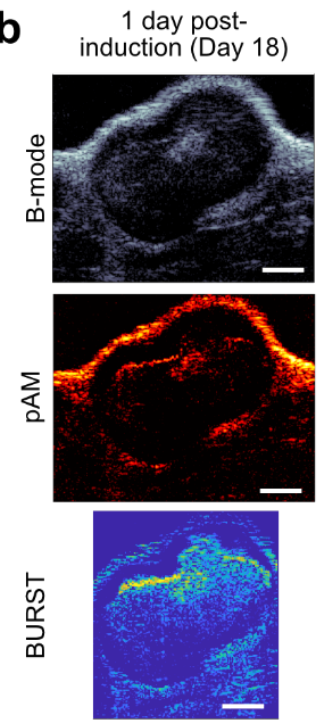

f

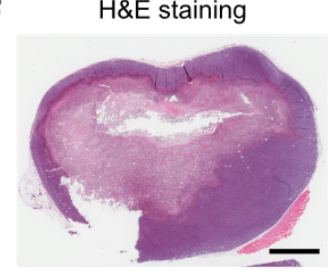

j

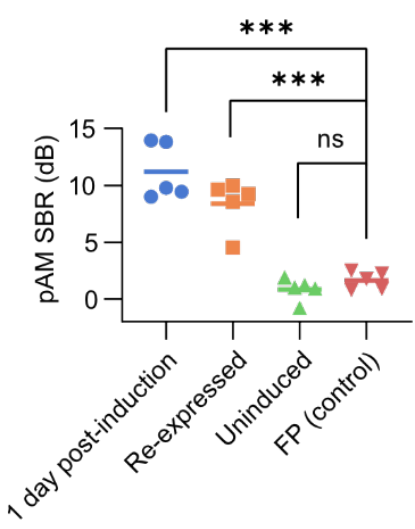

g
C Re-expressed
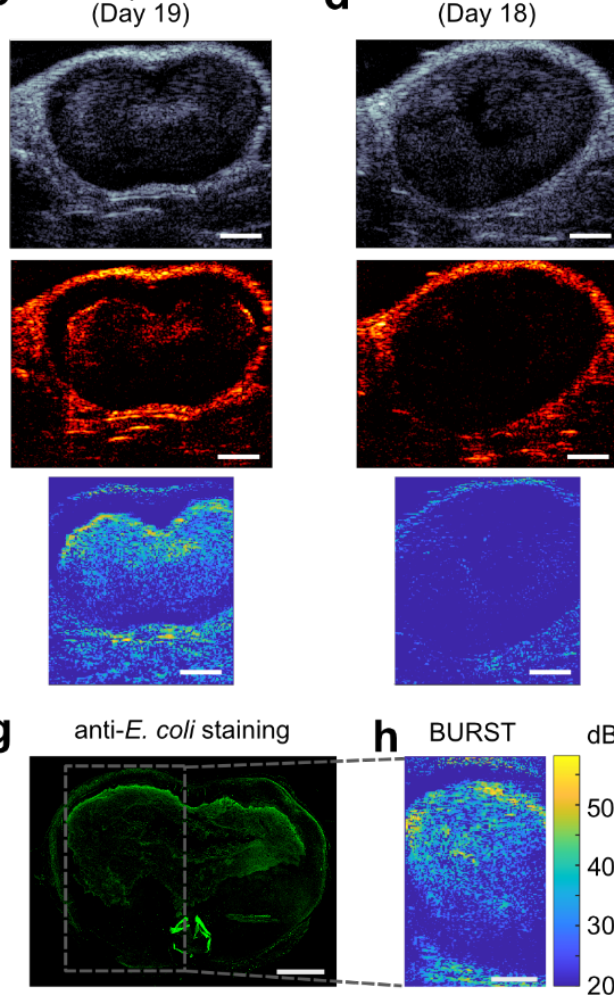

k
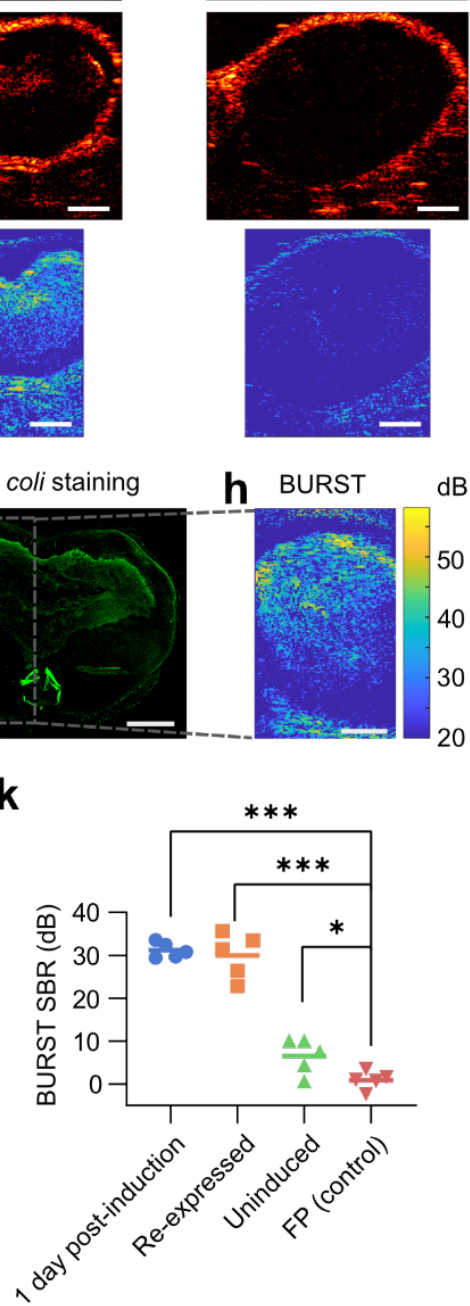

e
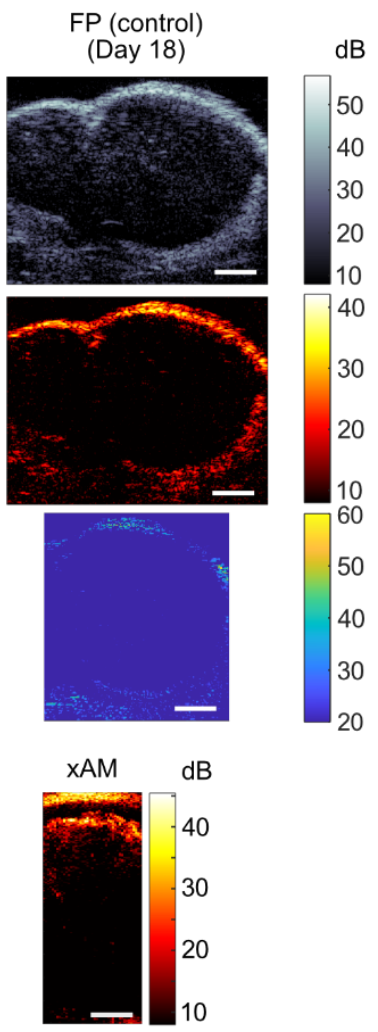

I

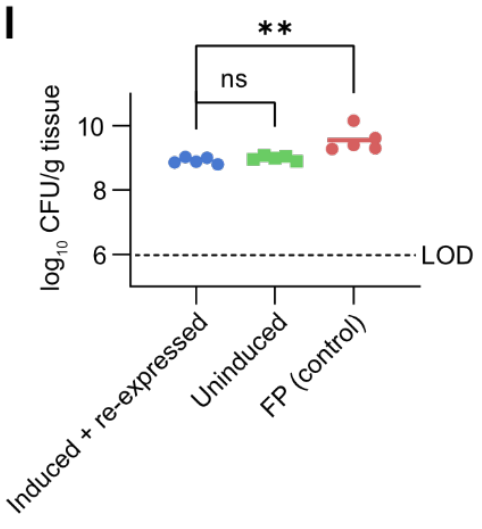




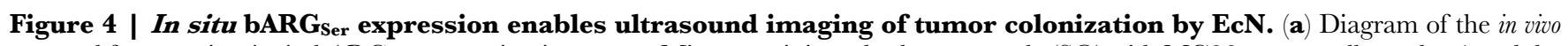
protocol for assessing in situ bARGser expression in tumors. Mice were injected subcutaneously (SQ) with MC26 cancer cells on day 1 and the tumors were allowed to grow for 14 days. Mice were then injected with $E$. coli Nissle (EcN) carrying either pBAD-bARGSer-AxeTxe or pBADFP-AxeTxe plasmids via the tail vein. After allowing 3 days for the EcN to colonize the tumors, bARGser or FP expression was induced by injecting L-arabinose intraperitoneally (IP) on day 17. The next day, at least 24 hours after induction, tumors were imaged with ultrasound. Subsequently, all the GVs in the tumors were collapsed by applying maximum acoustic pressure (3.0 MPa) throughout the tumor. L-arabinose was then injected again to re-induce bARGser expression, and tumors were again imaged with ultrasound at least 24 hours later. The next day (day 20), all mice were sacrificed, and their tumors were homogenized and plated on selective media to quantify the levels of EcN colonization. In separate experiments for histological analysis, mice were sacrificed on day 18 directly after ultrasound imaging. (b-d) Representative B-mode, parabolic AM (pAM), and BURST ultrasound images of tumors colonized by pBAD-bARGser-AxeTxe EcN at least 24 hours after induction with L-arabinose on day 18 (b), at least 24 hours after collapse and re-induction (day 19) (c), or uninduced on day 18 (d). (e) Representative B-mode, pAM, and BURST ultrasound images of tumors colonized by pBAD-FP-AxeTxe EcN at least 24 hours after induction with L-arabinose on day 18. (f-g) Optical images of tissue sections stained with H\&E (f) or anti-E. coli antibodies (g) from a tumor colonized by bARGser-expresssing EcN after ultrasound imaging on day 19. (h-i) BURST (h) and xAM (i) ultrasound images of the same tumor as in (f-g), with the boxed region showing the approximate BURST imaging region in the tissue section. Scale bars in (b-i) represent 2 mm. (j-k) Quantification of the pAM (j) and BURST (k) SBR for the same conditions in (b-e); each group is n=5 mice. (l) Log 10 of the colony forming units (CFUs) per gram of tissue from tumors homogenized and plated on day 20. The dotted line indicates the limit of detection (LOD). Asterisks represent statistical significance by unpaired t tests $(* * *=p<0.001, * *=p<0.01, *=p<0.05$, ns $=$ no significance). See Fig. $\mathbf{S 1 3}$ for representative xAM ultrasound images for the conditions in (b-d), and Fig. S14 for more histological images of tissue sections from tumors colonized with pBAD-bARGser-AxeTxe EcN.

colonized, ${ }^{27}$ as confirmed with subsequent tissue histology (Fig. 4f-i and S14).

Furthermore, after applying $3 \mathrm{MPa}$ acoustic pressure throughout the tumor to collapse all the GVs, re-injecting mice with L-arabinose inducer, and allowing $\geq 24$ hours for reexpression, similar ultrasound signals were observed in all

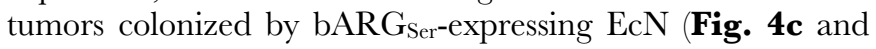
S13b). This result indicates that $b A R G_{\text {Ser }}$ can be used to visualize gene expression at multiple timepoints. Absent Larabinose induction, no xAM or pAM ultrasound signals were

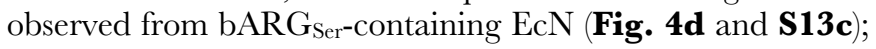
likewise, no xAM or pAM ultrasound signals were seen in tumors colonized by FP-expressing $\operatorname{EcN}(\mathbf{F i g} . \mathbf{4 e}, \mathbf{j}$ and S13de). Low levels of BURST signal were observed in uninduced animals (Fig. 4k), likely due to small amounts of L-arabinose present in the diet combined with BURST's high sensitivity.

To quantify tumor colonization, at the end of the experiment (day 20 in Fig. 4a) all mice were euthanized and their tumors were homogenized and plated on selective media. Tumors from all groups of mice $(n=5$ for induced and reexpressed $b A R G_{\text {Ser }}, n=5$ for uninduced $b A R G_{\text {Ser }}$, and $n=5$ for FP) contained more than $7 \times 10^{8} \mathrm{CFU} / \mathrm{g}$ tissue (Fig. 41), indicating that the $\mathrm{EcN}$ can persist at high levels in tumors for at least 6 days after IV injection regardless of bARG expression, collapse, and re-expression. The somewhat higher density of FP-expressing EcN suggested that maintenance of the smaller pBAD-FP-AxeTxe plasmid $(7.2 \mathrm{~kb}$ versus $23.2 \mathrm{~kb}$ for $\mathrm{pBAD}-\mathrm{bARG} \mathrm{G}_{\mathrm{Ser}}$-AxeTxe) may be easier in this in vivo context.

To test if there were any mutations causing a reduction in the ability of the cells to produce $\mathrm{GVs}$, tumors were plated on selective media with $0.1 \%$ L-arabinose and screened for colony opacity (Fig. S15a). Out of a total of 6,097 colonies screened from ten mice, only seven colonies were not opaque (Fig. S15b-c), suggesting a very low level of mutational silencing. Taken together, our in vivo experiments with $\mathrm{EcN}$ demonstrate that $\mathrm{bARG}_{\text {Ser }}$ expression enables stable, non-destructive acoustic visualization of the microscale distribution of these probiotic agents in a biotechnologyrelevant context.

\section{The A. flos-aquae $G$ V gene cluster produces robust nonlinear ultrasound contrast in mammalian cells.}

The first-generation mammalian ARGs were based on the GV gene cluster from B. megaterium (referred to here as $\mathrm{mARG}_{\mathrm{Mega}}$ ). $\mathrm{mARG}_{\mathrm{Mega}}$ expression in mammalian cells could only be detected with destructive collapse-based imaging due to their low levels of $\mathrm{GV}$ expression and the rigid shell of the resulting GVs. ${ }^{7}$ Seeking mARGs that produce nonlinear signal, we cloned mammalian versions of the genes contained in each of the three clusters that produced nonlinear signal in $E$. coli at $37^{\circ} \mathrm{C}$ : Serratia, A. flos-aquae, and A. flos-aquae/B. megaterium (Fig. 1d-e). Equimolar transient cotransfections of the monocistronic genes derived from each gene cluster into HEK293T cells yielded detectable BURST signal only for the A. flos-aquae gene cluster and the positive control $\mathrm{mARG}_{\mathrm{Mega}}{ }^{7}$ (Fig. 5a, 5b "61-fold excess").

Given the multiple gopA copies contained in the native A. flos-aquae $\mathrm{GV}$ operon, ${ }^{4}$ we hypothesized that expressing the major structural protein GvpA at a higher stoichiometry relative to the other genes in this cluster could improve GV expression. To test this hypothesis, we titrated the amount of gop $A$ plasmid in the $A$. flos-aquae plasmid set, while keeping the DNA amount corresponding to other genes constant the total DNA level was also kept constant with a padding vector). We found that the BURST signal increased monotonically with increasing gvpA up to 8-fold gvpA excess. In contrast, the signal peaked at 2-fold excess of the structural protein $g * p B$ when expressing the $B$. megaterium cluster (Fig. 5b). To further improve GvpA expression, we stabilized the gopA transcript with WPRE-hGH poly(A) elements, ${ }^{35}$ which resulted in peak signal at lower gopA ratios (Fig. S16a-b).

We next looked for nonlinear ultrasound contrast from A. flos-aquae $\mathrm{GVs}$ to enable their detection versus background. GvpC is a minor structural protein in A. flos-aquae GVs that binds to and mechanically reinforces the GV shell (Fig. 5c). ${ }^{4}$ In vitro chemical removal of GvpC from purified 
bioRxiv preprint doi: https://doi.org/10.1101/2021.04.26.441537; this version posted October 29, 2021. The copyright holder for this preprint (which was not certified by peer review) is the author/funder. All rights reserved. No reuse allowed without permission.

a

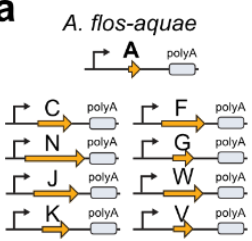

B. megaterium $\Gamma \mathrm{B}$ polyA

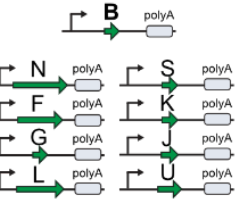

C

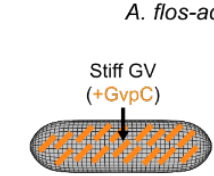

A. flos-aquae GVs

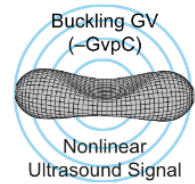

e

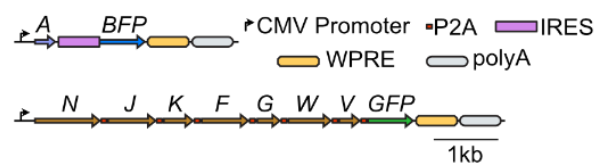

b
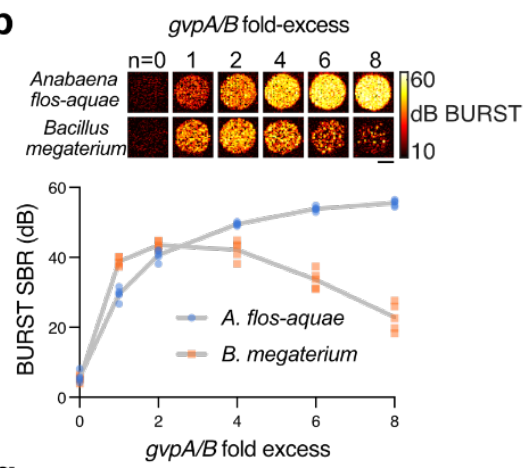

g

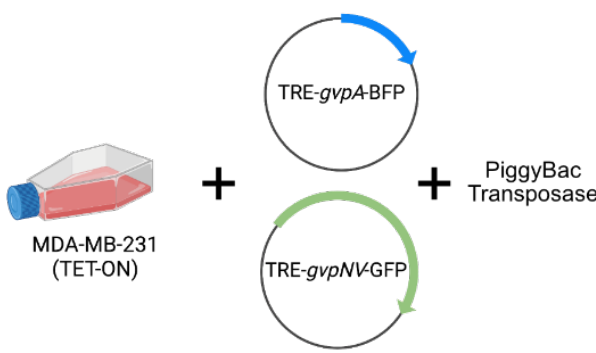

h
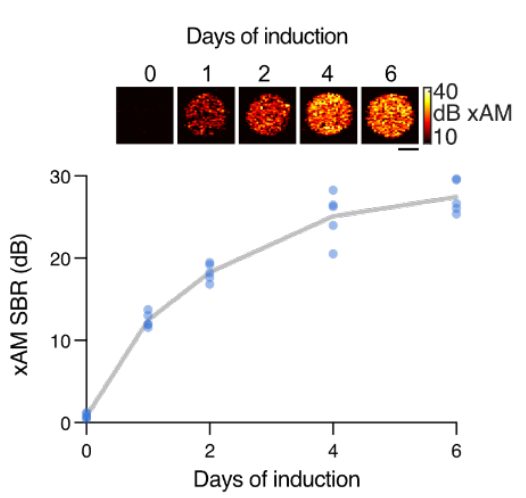

i d
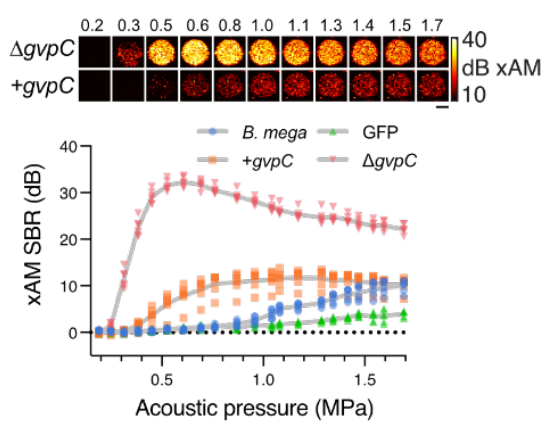

f
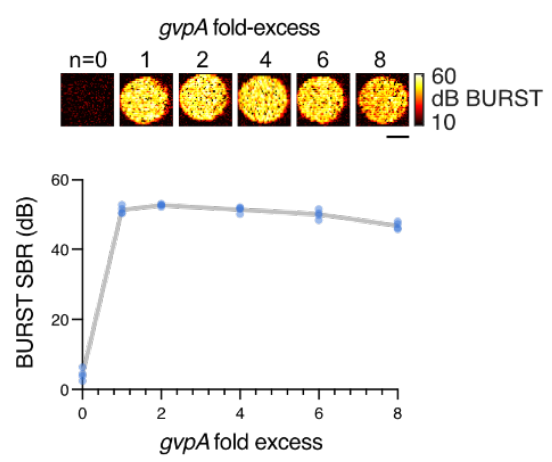

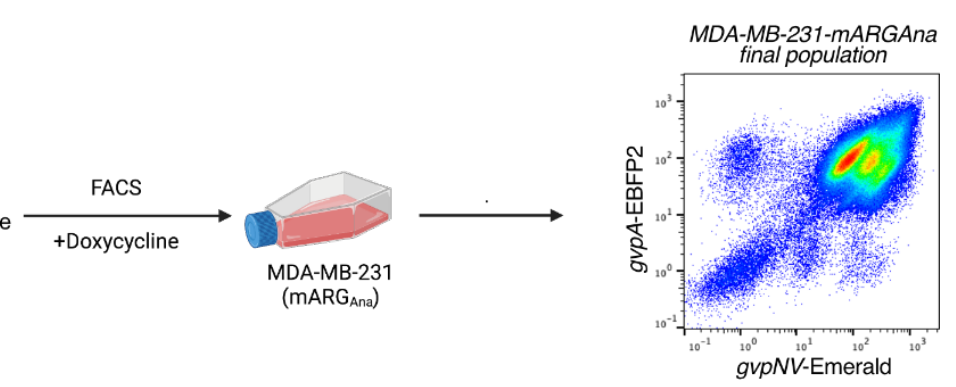

Doxycycline Concentration $(\mu \mathrm{g} / \mathrm{mL})$
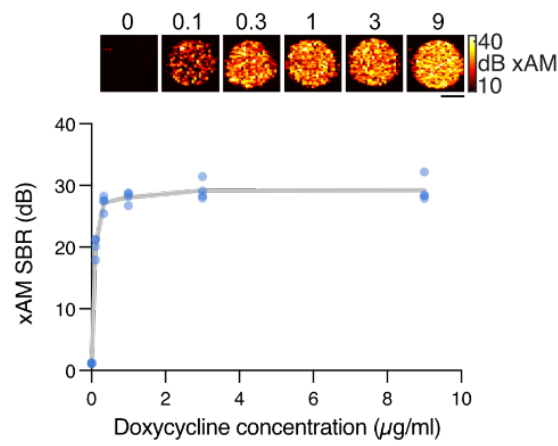

j

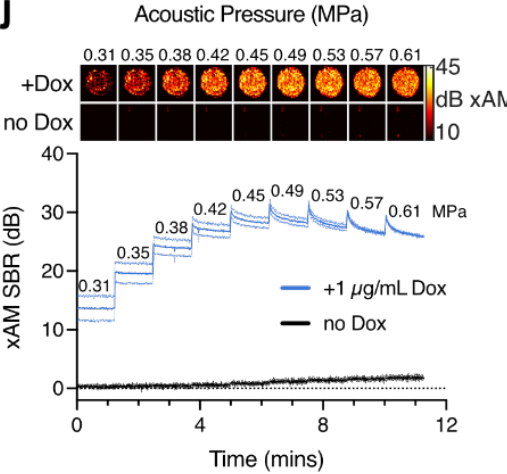

Figure 5 |Heterologous expression of the A. flos-aquae GV gene cluster in mammalian cells. (a) Schematic of the codonoptimized monocistronic plasmid sets used in this study. (b) Representative BURST images (top) and SBR quantification ( $\mathrm{n}=5$, bottom) of transient GV expression in HEK293T cells 3 days after cotransfection of mixtures with varying gop $A / B$ fold excess relative to their respective assembly factor plasmids. (c) Diagram of GV structure with GvpC highlighted in orange. (d) Representative xAM ultrasound images (top) and SBR quantification ( $\mathrm{n}=6$, bottom) of transient cotransfection experiments of $A$. flos-aquae $\mathrm{GV}$ plasmids (4-fold gopA excess) with and without gop $C$ at varying acoustic pressures. B. megaterium $\mathrm{GV}$ (at 2-fold gopB excess) and GFP expression is included for quantitative comparison. (e) Schematic of the $\mathrm{mARG}_{\text {Ana }}$ plasmid set with fluorescent reporters. (f) Representative BURST images (top) and SBR quantification (n=4,

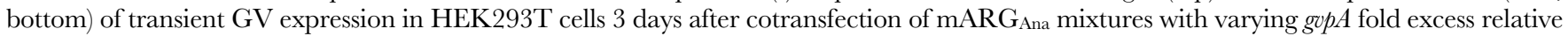

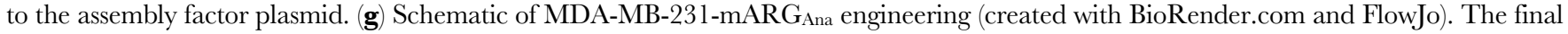
population was $\sim 95 \%$ double positive for gvpA and gvp $N 7 K F G W V$ expression. (h) Representative xAM images (top) and SBR quantification ( $\mathrm{n}=5$, bottom) of MDA-MB-231-mARG $\mathrm{mna}_{\text {a }}$ cells at $0.54 \mathrm{MPa}$ after $1,2,4$ and 6 days of $1 \mu \mathrm{g} / \mathrm{ml}$ doxycycline induction. (i) Representative xAM images (top) and SBR quantification ( $\mathrm{n}=4$, bottom) of MDA-MB-231-mARGAna cells at $0.42 \mathrm{MPa}$ as a function of doxycycline concentration after 4 days of expression. (j) Representative xAM images (top) and SBR quantification ( $\mathrm{n}=4$, bottom) of induced and uninduced MDA-MB-231-mARG Ana cells as a function of time under varying acoustic pressures. For $\mathrm{j}$, thick lines represent the mean of 4 replicates and thin lines represent \pm standard deviation. For b, d, f, h, and i, gray lines connect the means of the replicates. All ultrasound image scalebars represent $1 \mathrm{~mm}$. 
GVs was previously shown to enhance nonlinear ultrasound scattering by allowing the GVs to deform more strongly and nonlinearly in response to acoustic pressure. . $^{14,36,37}$ When we omitted gopC from our transient cotransfection mixture, we observed a dramatic enhancement of nonlinear signal in xAM imaging, with the peak signal produced at around 0.6 MPa. By comparison, the transfection mixture including gv $C$ produced a much weaker xAM signal, while the $B$. megaterium plasmid mixture and GFP-expressing cells did not produce appreciable nonlinear contrast at any pressure (Fig. 5d). The omission of gop $C$ did not appreciably alter BURST contrast (Fig. S16c). These results indicated that the mammalian GVs derived from A. flos-aquae can provide nondestructive nonlinear ultrasound contrast.

To create a convenient vector for mammalian expression of A. flos-aquae $\mathrm{GVs}$, we constructed a polycistronic plasmid linking the assembly factor genes gupN $7 K F G W V$ through P2A self-cleaving peptides. gopA was supplied on a separate plasmid to enable stoichiometric tuning. The gopA and gopN $7 K F G W V$ plasmids were labeled with IRES-BFP and P2AGFP, respectively, to allow for fluorescently activated cell sorting (FACS). Both plasmids were driven by CMV promoters, and their transcripts were stabilized with WPREhGH poly(A) elements. We termed this pair of plasmids $\mathrm{mARG}_{\text {Ana }}$ - mammalian ARGs adapted from A. flos-aquae (Fig. 5e). mARG $\mathrm{Ana}_{\text {Ana }}$ produced robust $\mathrm{GV}$ expression and ultrasound contrast in HEK293T cells with a 1- to 6-fold excess of $g v p A$ (Fig. 5f).

One of the most common applications of mammalian reporter genes is the visualization of tumor growth in animal models of cancer, which are a critical platform for basic oncology research and the development of new treatments. To produce a stable cancer cell line expressing $\mathrm{mARG}_{\text {Ana, }}$, we cloned our polycistronic constructs into PiggyBac integration plasmids under a doxycycline-inducible TRE promoter. ${ }^{38,39} \mathrm{As}$ a clinically relevant cancer model, we chose the human breast cancer cell line MDA-MB-231, which is widely used in xenograft studies. We engineered these cells to constitutively co-express the rtTA transactivator and Antares optical reporter ${ }^{40}$, then electrically transduced them with a mixture of $\mathrm{mARG}_{\text {Ana }}$ and PiggyBac transposase plasmids at a 2:1 (gvpA:gvpNfKFGWV) molar ratio. We used FACS to sort for coexpression of Antares, GFP and BFP (Fig 5g, S16d).

The resulting polyclonal MDA-MB-231-mARG $\mathrm{Ana}_{\text {Ana }}$ cells produced GVs detectable by TEM and showed xAM contrast after a single day of doxycycline induction, which increased substantially through day 6 (Fig. 5h, S16e). The signal increased steeply with increasing doxycycline doses up to $1 \mu \mathrm{g} / \mathrm{mL}$, above which the signal saturated (Fig. 5i). xAM was detected from induced cells starting from an acoustic pressure of $0.31 \mathrm{MPa}$, whereas uninduced control cells did not produce signal at any pressure. In a ramp of acoustic pressure, the xAM signal gradually decreased over time at pressures above $0.42 \mathrm{kPa}$, indicating partial collapse of GVs $(\mathbf{F i g}$. 5j). We chose $0.42 \mathrm{MPa}$ as the xAM imaging pressure for subsequent experiments, providing the optimal combination of signal stability and signal strength. $m A R G_{A n a}$ expression enables visualization of in vivo gene expression patterns in an orthotopic tumor model. We next tested the ability of $\mathrm{mARG}_{\mathrm{Ana}}$ to image the spatial distribution of gene expression in tumor xenografts in living mice. We formed orthotopic tumors by injecting MDAMB-231-mARG $\mathrm{Ana}_{\text {a }}$ cells bilaterally in the fourth mammary fat pads of female immunocompromised mice. The mice were then split into doxycycline-induced and uninduced groups. We acquired ultrasound images of the tumors using xAM every 4 days for a total of 3 imaging sessions (Fig. 6a). All induced tumors produced bright and specific xAM contrast starting from the first day of imaging, whereas the uninduced tumors did not (Fig. 6b). The acquisition of adjacent planes allowed 3D visualization of expression patterns (Supplementary

Video 1). The nonlinear xAM signal was highly specific to the viable tumor cells, being absent outside the anatomically visible tumor boundaries and within the tumor cores at 8 and 12 days. The observed spatial pattern of gene expression activity in these tumors was corroborated by fluorescence microscopy of formalin-fixed tumor sections obtained from euthanized mice on day 12 (Fig. 6c, Fig. S17), confirming the ability of $\mathrm{mARG}_{\mathrm{Ana}}$ to report microscale patterns of gene expression noninvasively in intact animals. In contrast, in vivo fluorescence images of the mice lacked information about the spatial distribution of gene expression within the tumor $(\mathbf{F i g} .6 \mathbf{6 d})$. GV expression over time was quantified as a volumetric sum of xAM signal over all acquired image planes (Fig. 6e). The induced tumors had significantly higher total signal than the uninduced controls at all time points. These experiments demonstrate the ability of $\mathrm{mARG}_{\text {Ana }}$ to serve as a highly effective reporter gene for the noninvasive imaging of tumor growth and gene expression activity.

\section{DISGUSSION}

In sum, our results establish two second-generation ARG constructs - bARG $\mathrm{ber}_{\text {er }}$ and $\mathrm{mARG}_{\mathrm{Ana}}$ - that provide unprecedented US detection sensitivity and specificity when expressed in probiotic bacteria and mammalian cells. These gene clusters, obtained through a systematic phylogenetic screen and optimized through genetic engineering, produce bright nonlinear US signal ${ }^{14}$ when expressed in situ in tumors, either by bacterial agents colonizing the necrotic core of a tumor, or by the tumor cells themselves. When imaged using a highly sensitive and specific non-destructive US imaging paradigm, this nonlinear signal enables real-time monitoring of the precise locations and transcriptional activity of these cells and is sufficiently stable to image cellular biodistribution and gene expression over multiple days.

These results comprise major improvements over previous work on heterologous GV expression in bacterial and mammalian cells. Previous bARG constructs required the host bacteria to be cultured and pre-express GVs under ideal laboratory conditions before in vivo injection, ${ }^{6}$ while the previous mARG construct only produced ultrasound contrast when expressed in a sorted monoclonal cell line. ${ }^{7}$ In both cases, sensitive and specific detection of these cells relied on destructive imaging methods that produce one-time contrast, 
bioRxiv preprint doi: https://doi.org/10.1101/2021.04.26.441537; this version posted October 29, 2021. The copyright holder for this preprint (which was not certified by peer review) is the author/funder. All rights reserved. No reuse allowed without permission.

a

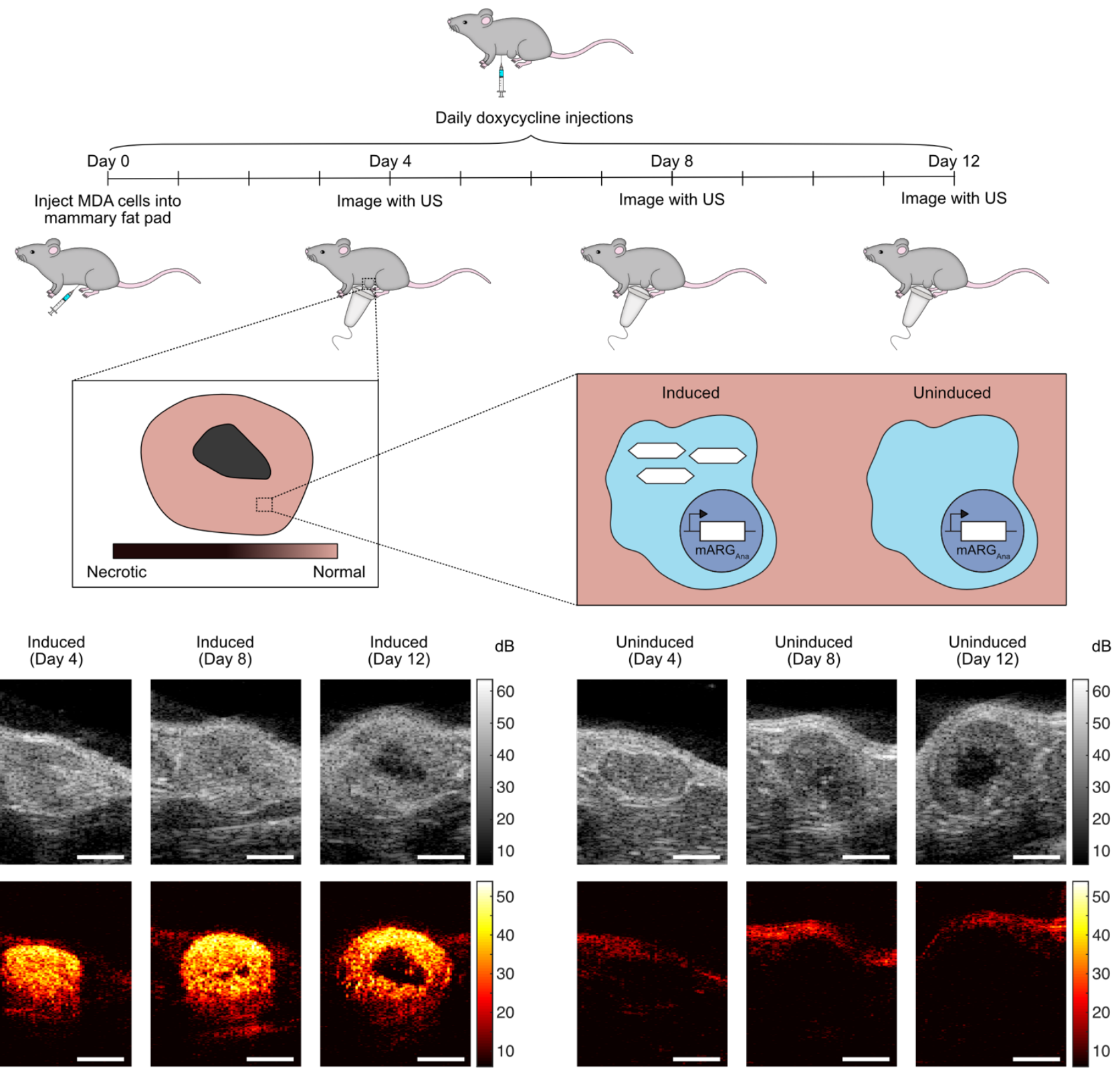

C
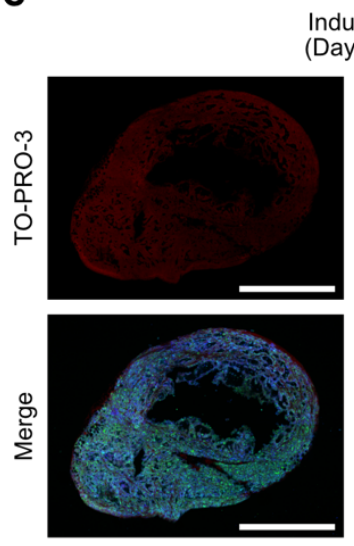

Induced Day 12)
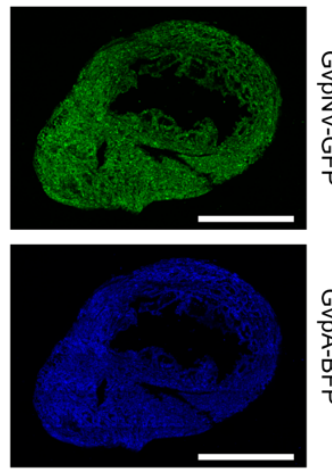

d

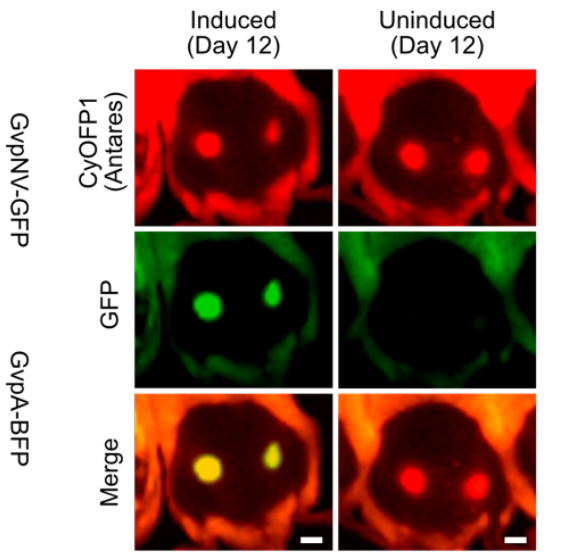

e

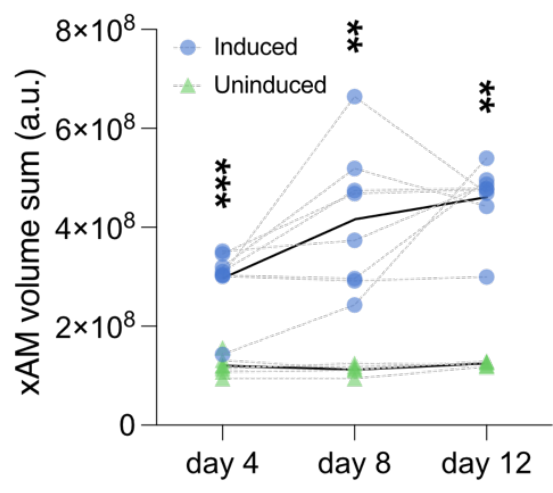




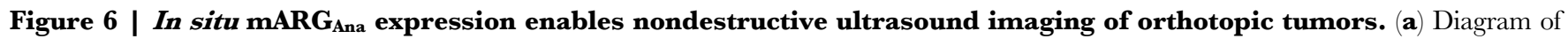
the in vivo protocol for assessing in situ $\mathrm{mARG}_{\text {Ana }}$ expression in orthotopic tumors. Mice were injected bilaterally in $4^{\text {th }}$ mammary fat pads with engineered MDA-MB-231-mARGAna human breast adenocarcinoma cells on day 0. mARGAna expression was induced by regular intraperitoneal (IP) doxycycline injections starting from the day of tumor injections. Tumors were imaged with ultrasound after 4, 8 and 12 days of expression. (b) Representative middle sections of B-mode and xAM ultrasound tomograms of MDA-MB-231 mARG induced with doxycycline (left) and uninduced control (right) imaged on day 4, 8 and 12. Scalebars represent 2 mm. See supplementary video for the full ultrasound tomogram of the induced tumor at 12 days. (c) Fluorescence micrograph of a $100 \mathrm{~nm}$ thin tumor section. Green color shows GFP fluorescence, blue color shows BFP fluorescence, and red color shows TO-PRO-3 nuclear stain. See Fig. S17 for the uninduced control. Scalebars represent $2 \mathrm{~mm}$. (d) Whole-animal fluorescence imaging of induced (left) and uninduced (right) mouse after 12-days of

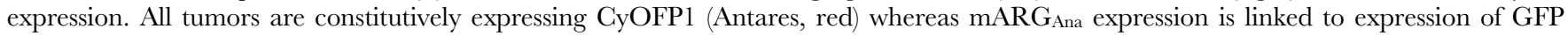
(green). The left (reader's right) tumors are shown in panel (b). Scalebars represent $5 \mathrm{~mm}$. (e) Three-dimensional sum of xAM signal from ultrasound tomograms of induced $(n=8)$ and uninduced $(n=7$ on day $4, n=5$ on days 8 and 12) tumors from all three imaging sessions plotted on a linear scale in arbitrary units. Asterisks represent statistical significance by unpaired t tests between induced and uninduced conditions $(* * *=\mathrm{p}<0.001, * *=\mathrm{p}<0.01, *=\mathrm{p}<0.05$, ns $=$ no significance $)$.

hindering their use for monitoring dynamic biological processes. ${ }^{8}$

With these major improvements, we anticipate that these new ARGs will be useful for many applications that demand the long-term, noninvasive imaging of cells deep inside the body. bARGs could be used to track therapeutic bacteria as they either home to and attack tumors, or travel through the gastrointestinal (GI) tract and report on its inflammatory state. ${ }^{41,42} \mathrm{mARG}$ could be used to visualize the growth of a viability of tissues such as tumors, or monitor select cell types or cellular states in a tissue using selective promoters. Further, A. flos-aquae GVs have proven to be a versatile engineering platform, $2,6,36,43$ and the nonlinear signal generation of both ARGs mean that they could form the basis for acoustic biosensors of more dynamic cellular signals such as enzyme activity. 43

While these results represent an important step in the development of ARGs, additional improvements could further expand their utility in biotechnology. First, the expression kinetics of ARGs are slower than those of fluorescent proteins in E. coli ${ }^{44}$ and mammalian cells, ${ }^{45}$ and faster expression would facilitate the imaging of more dynamic genetic outputs. Second, the ability to multiplex different "colors" of ARGs nondestructively in vivo would enable discrimination between different strains of engineered bacteria in a consortium, ${ }^{46-48}$ between different mammalian cell types in a tissue, or between bacterial and mammalian cells in close proximity, such as in a bacterially-colonized tumor. Additionally, the Serratia GV gene cluster is relatively large, making it more challenging to clone and incorporate with other genetic elements. The engineering of a shorter cluster with similar acoustic properties would simplify these efforts. Similarly, the current $\mathrm{mARG}_{\text {Ana }}$ cluster is delivered using two polycistronic plasmids; consolidating this cluster into a single plasmid would increase transfection efficiency, and shortening it could enable its viral packaging and delivery to endogenous cells in situ.

Our phylogenetic screening approach was successful in identifying bARG ${ }_{\text {Ser }}$ and $m A R G_{\text {Ana }}$ as greatly improved ARGs. However, out of practical necessity, this screen subsampled the available phylogenetic space, and thus testing additional GV-encoding gene clusters could reveal ARGs with new or further-improved properties. Improvements can also be made in the screening strategy. It is challenging to identify all the gop genes in a given genome (see Methods), and there is considerable regulation of GV cluster transcription by factors inside $^{5,17}$ or outside ${ }^{18}$ the clusters. Therefore, in a given cloned cluster, it is possible that either essential genes are missing or cryptic regulatory elements are included.5,15,19 These issues could be resolved by synthesizing multiple versions of each putative gene cluster and screening a larger number of them in higher throughput. Even with optimal genetic constructs, it is likely that gene clusters from some species will not successfully form GVs in a given heterologous host due to differences in growth temperature, turgor pressure or the presence or absence of specific host factors. The phylogenetic screening strategy used in this study could thus be adapted to find optimal ARGs for use in other species of interest.

Just as improvements to and adaptations of fluorescent proteins enabled a wide range of microscopy applications that were mere speculations when GFP was first harnessed as a biotechnology, the systematic development of next-generation ARGs will help bring to reality the promise of sensitive, high-resolution noninvasive imaging of cellular function inside intact mammals.

\section{MATERIALS AND METHODS}

\section{Genomic mining of ARG clusters}

A literature search was conducted to find papers reporting the production of gas vesicles in any species. Search terms included "gas vesicle," "gas vacuole," "aerotope," and "aerotype." All species found are listed in Table S1. If the report did not include a strain name, then any available 16S rRNA gene sequence was used (as it was assumed that any other strain of the same species would fall in the same place on the phylogenetic tree), but no GV gene cluster sequence was used (even if it was available for one or more strains of that species) because it was found during our analysis that: 1) several reports describe species for which some strains produce GVs but others don't, and 2) comparison of GV gene cluster sequences of multiple strains of the same species almost always showed differences - often very significant ones. Further, even if a reference stating that a given organism produced GVs was not available, 16S rRNA gene sequences from all members of the following genera were included because GV production is a diacritical taxonomic feature for these genera: Dolichospermum, ${ }^{49}$ Limnoraphis ${ }^{50}$ and Planktothrix. ${ }^{51}$

GV clusters were identified in genomes through a combination of annotations and sequence similarity to known 
gop genes. However, there were two challenges in identifying all gops in a given genome: 1) there is little to no annotation for many gops, and 2) GV gene clusters are not always contiguous in genomes, and gops can occasionally be found hundreds of $\mathrm{kb}$ away from the main cluster(s). We attempted to only select "well-behaved" GV clusters for testing (i.e., ones in which all gops identified in that genome were present in clusters, and these clusters contained a minimum of non-grp genes, which could increase the metabolic burden of cluster expression without increasing GV yield), but it is possible that even for these clusters, some grps were not cloned.

Of our list of 288 strains reported to form gas vesicles, 270 had 16S rRNA gene sequences available (Table S1). These were downloaded from NCBI using a custom Python script, and a multiple sequence alignment was constructed using Clustal Omega. ${ }^{52}$ This alignment was used to generate a phylogenetic tree file using ClustalW2, ${ }^{33}$ which was rendered using EvolView. ${ }^{54}$ Only unique species are displayed in the phylogenetic trees in Fig. 1a and Fig. S1.

\section{Bacterial plasmid construction and molecular biology}

Organisms were obtained from commercial distributors as indicated in Table S2. If an organism was shipped as a liquid culture, the culture was centrifuged and the pellet resuspended in $\mathrm{ddH} 2 \mathrm{O}$, as it was found that even trace amounts of certain culture media could completely inhibit PCR. Fragments were amplified by PCR using Q5 polymerase and assembled into a pET28a(+) vector (Novagen) via Gibson Assembly using reagents from New England Biolabs (NEB). Sub-cloning and other modifications to plasmids were also performed with Gibson Assembly using reagents from NEB. Assemblies were transformed into NEB Stable E. coli. All constructs were verified by Sanger sequencing.

Halobacterium salinarum has two chromosomal GV gene clusters (plus additional plasmid-borne ones), which were cloned and tested separately. Methanosarcina vacuolata has only one cluster, but while its genome sequence in the NCBI database has two copies of GvpA1 and one copy of GvpA2, our genomic PCR yielded a product with only one copy of GupA1. In a second cloning step, we added a copy of GupA2 to the cloned cluster. While we were able to PCR GvpA2 from the genome, it was not contiguous with the rest of the cluster. Therefore, we speculate that either there was an error in the assembly of the genome sequence (likely caused by the high sequence similarity of the GupA genes), or that the genotype of our strain differs slightly from that of the strain sequenced.

\section{In vitro bacterial expression of ARGs}

For initial testing, all constructs were expressed in BL21(DE3) $E$. coli (NEB). Fifty $\mu \mathrm{L}$ of electrocompetent $E$. coli were transformed with $1.5 \mu \mathrm{L}$ of purified plasmid DNA (Econospin 96-well filter plate, Epoch Life Science), and $1 \mathrm{~mL}$ of SOG medium (NEB) was added immediately after electroporation. These cultures were incubated at $37^{\circ} \mathrm{C}$ for $2 \mathrm{hr}$, and $150 \mathrm{uL}$ was inoculated into larger $1.5 \mathrm{~mL} \mathrm{LB}$ cultures containing 100 $\mathrm{ug} / \mathrm{mL}$ kanamycin and $1 \%(\mathrm{w} / \mathrm{v})$ glucose (for catabolite repression ${ }^{55}$ of the BL21(DE3) PlacUV5 promoter) in a deepwell 96-well plate and grown overnight in a shaking incubator at $30^{\circ} \mathrm{C}$. Square dual-layer LB agar plates were prepared as described previously, ${ }^{6}$ with varying concentrations of IPTG and $100 \mathrm{ug} / \mathrm{mL}$ kanamycin in the bottom layer, and 1\% (w/v) glucose and $100 \mathrm{ug} / \mathrm{mL}$ kanamycin in the top layer. LB agar was incubated at $60^{\circ} \mathrm{C}$ for $12-36 \mathrm{hr}$ after dissolution to allow it to degas. After the agar solidified, plates were dried at $37^{\circ} \mathrm{C}$ to remove all condensation on the top layer that would cause the bacterial patches to run together. A multichannel pipette was used to thoroughly mix overnight cultures and drop $1 \mu \mathrm{L}$ of each culture onto the surface of the dual-layer plates, with care taken to avoid puncturing the agar which results in artifacts during ultrasound scans. Importantly, low-retention pipette tips were used, as it was found that the small volumes of culture would wet the outsides of standard pipette tips, resulting in inaccurate volume dispensing. Patches were allowed to dry completely before plates were flipped and incubated at $37^{\circ} \mathrm{C}$ for $24 \mathrm{hr}$ or $30^{\circ} \mathrm{C}$ for $48 \mathrm{hr}$.

For in vitro expression experiments in $\mathrm{EcN}$, the appropriate plasmids were first transformed via electroporation and the outgrowth was plated on LB (Miller)agar plates with the appropriate antibiotic $(25 \mu \mathrm{g} / \mathrm{mL}$ chloramphenicol or $50 \mu \mathrm{g} / \mathrm{mL}$ kanamycin) and $1 \%(\mathrm{w} / \mathrm{v})$ glucose. The resulting colonies were used to inoculate $2 \mathrm{~mL} \mathrm{LB}$ (Miller) medium with the appropriate antibiotic and 1\% (w/v) glucose, and these cultures were incubated at $250 \mathrm{rpm}$ and $37^{\circ} \mathrm{C}$ overnight. Glycerol stocks were prepared by mixing the overnight cultures in a 1:1 volume ratio with $50 \%(\mathrm{v} / \mathrm{v})$ glycerol and storing at $-80^{\circ} \mathrm{C}$. The night before expression experiments, glycerol stocks were used to inoculate overnight cultures $(2 \mathrm{~mL} \mathrm{LB}$ medium with the appropriate antibiotic and $1 \%(\mathrm{w} / \mathrm{v})$ glucose $)$ which were incubated at $37^{\circ} \mathrm{C}$ and shaken at $250 \mathrm{rpm}$. For expression on solid media, $1 \mu \mathrm{L}$ of overnight culture was dropped onto square dual-layer LB agar plates with 2X the final inducer (IPTG, aTc, or L-arabinose) concentration in the bottom layer, $1 \%(\mathrm{w} / \mathrm{v})$ glucose in the top layer, and the appropriate antibiotic in both layers $(50 \mu \mathrm{g} / \mathrm{mL}$ chloramphenicol or $100 \mu \mathrm{g} / \mathrm{mL}$ kanamycin). Plates were allowed to dry, and then inverted and incubated at $37^{\circ} \mathrm{C}$ for 24 hours before imaging with ultrasound. For expression in liquid media, $500 \mu \mathrm{L}$ of each overnight culture was used to inoculate $50 \mathrm{~mL} \mathrm{LB}$ supplemented with $0.4 \%(\mathrm{w} / \mathrm{v})$ glucose and 25 $\mu \mathrm{g} / \mathrm{mL}$ chloramphenicol in $250 \mathrm{~mL}$ baffled flasks. Cultures were incubated at $37^{\circ} \mathrm{C}$ and $250 \mathrm{rpm}$ until reaching at $\mathrm{OD}_{600}$ of $0.1-0.3$. At this point, cultures were induced by addition of L-arabinose and placed back at $37^{\circ} \mathrm{C}$ and $250 \mathrm{rpm}$. For time titration experiments, $0.1 \%(\mathrm{w} / \mathrm{v})$ L-arabinose was used for induction and $0.5 \mathrm{~mL}$ of each culture was removed at $0,1,3$, $5,7,9,11$, and 24 hours post-induction for $\mathrm{OD}_{600}$ and ultrasound measurements. For L-arabinose titration experiments, $\mathrm{L}$-arabinose concentrations ranging from 0 to $1 \%$ $(\mathrm{w} / \mathrm{v})$ were used for induction, and cultures were incubated for 24 hours at $37^{\circ} \mathrm{C}$ and $250 \mathrm{rpm}$ after addition of L-arabinose before ultrasound imaging. For cell concentration titration experiments, cultures were incubated for 24 hours at $37^{\circ} \mathrm{C}$ and $250 \mathrm{rpm}$ after addition of $0.1 \%(\mathrm{w} / \mathrm{v})$ L-arabinose before ultrasound imaging. All cultures were stored at $4^{\circ} \mathrm{C}$ or on ice until casting in phantoms and imaging with ultrasound. In all liquid culture experiments, cultures were prescreened for the 
presence of GVs by phase contrast microscopy before being imaged with ultrasound.

To assess plasmid stability of $\mathrm{pBAD}^{-b A R \mathrm{SSer}^{-}}$ AxeTxe in EcN, the glycerol stock of this strain was used to inoculate $2 \mathrm{~mL}$ LB (Miller) supplemented with $25 \mu \mathrm{g} / \mathrm{mL}$ chloramphenicol and $1 \%(\mathrm{w} / \mathrm{v})$ glucose, and this culture was incubated at $37^{\circ} \mathrm{C}$ and $250 \mathrm{rpm}$ overnight. Twenty $\mu \mathrm{L}$ of the overnight culture was subcultured into $2 \mathrm{~mL} \mathrm{LB}$ with 25 $\mu \mathrm{g} / \mathrm{mL}$ chloramphenicol, $2 \mathrm{~mL}$ LB without antibiotics, and 2 $\mathrm{mL}$ LB without antibiotics and with $0.1 \%(\mathrm{w} / \mathrm{v})$ L-arabinose, each in quadruplicate. Every 24 hours, $20 \mu \mathrm{L}$ of each culture was sub-cultured into fresh media of the same conditions. All cultures were incubated at $37^{\circ} \mathrm{C}$ and $250 \mathrm{rpm}$. On days $1-3,5$, and 7, serial dilutions of each culture were plated on LB-agar without antibiotics, $\mathrm{LB}$-agar with $25 \mu \mathrm{g} / \mathrm{mL}$ chloramphenicol, and LB-agar with $25 \mu \mathrm{g} / \mathrm{mL}$ chloramphenicol $+0.1 \%(\mathrm{w} / \mathrm{v})$ L-arabinose $+0.4 \%(\mathrm{w} / \mathrm{v})$ glucose. Plates were incubated at $37^{\circ} \mathrm{C}$ for at least 16 hours and colonies were counted and screened manually. Plasmid retention was assessed by taking the ratio of CFUs on LB-agar plates with chloramphenicol to CFUs on LB-agar plates without antibiotics. The presence of mutations that disrupt the ability to express functional bARG $_{\text {Ser }}$ was assessed by a loss of colony opacity on LB-agar plates with $25 \mu \mathrm{g} / \mathrm{mL}$ chloramphenicol $+0.1 \%(\mathrm{w} / \mathrm{v}) \mathrm{L}$ arabinose $+0.4 \%(\mathrm{w} / \mathrm{v})$ glucose.

In vitro ultrasound imaging of bacteria expressing ARGs on solid media Ultrasound imaging of bacterial patches was performed using a Verasonics Vantage programmable ultrasound scanning system and an L10-4v 128-element linear array transducer (Verasonics) with a center frequency of $6 \mathrm{MHz}$ and an element pitch of $300 \mu \mathrm{m}$. Image acquisition was performed using a custom imaging script with a 64-ray-lines protocol and a synthetic aperture of 65 elements. The transmit waveform was set to a voltage of $50 \mathrm{~V}$ and a frequency of $10 \mathrm{MHz}$, with 1 waveform cycle and $67 \%$ intra-pulse duty cycle. In xAM mode, a custom sequence detailed previously ${ }^{14}$ was used with an angle of $19.5^{\circ}$. RF data from 4 repeated acquisitions was coherently averaged prior to beamforming for each image plane.

Agar plates containing bacterial patches were coated with a thin layer of LB agar and immersed in PBS to allow acoustic coupling to the L10-4v transducer. The transducer was connected to a BiSlide computer-controlled 3D translatable stage (Velmex) and positioned above the plane of the plate at an angle of $15^{\circ}$ from the vertical (to minimize specular reflection from the plastic dishes and agar) and a distance of $20 \mathrm{~mm}$ from the bacterial patches. The imaging sequence was applied sequentially to acquire image planes covering the full area of all plates. A custom script was used to automate the scan by controlling the motor stage in tandem with the ultrasound system, translating $0.5 \mathrm{~mm}$ in the azimuthal direction between rows and $19.5 \mathrm{~mm}$ in the lateral direction between columns. In the case of pre-minus-postcollapse scans, the full scan sequence was repeated after returning the motor stage to its origin and adjusting the voltage of the transducer.

For image processing and analysis, custom beamforming scripts were applied on-line to reconstruct image planes from the acquired RF data at each location. The intensity data for each plane was saved for off-line processing. All image planes were concatenated to form a 3D volume with all plates and colonies. A 2D image of the colonies was extracted from the 3D volume by taking the maximum intensity over a manually-defined depth range for all voxel columns. 2D pre-minus-post-collapse images were obtained by subtracting the post-collapse 2D image from the pre-collapse 2D image. Bacterial patch intensities were then quantified from these 2D images. Sample ROIs were drawn around the center of each patch to avoid artefacts from the edges, and background ROIs were drawn around representative regions without patches. The signal-to-background ratio (SBR) was calculated as the mean pixel intensity of the sample ROI divided by the mean pixel intensity of the background. Conversion to decibels $(\mathrm{dB})$ was calculated as $20 * \log 10(\mathrm{SBR})$. For display, images were normalized by dividing by the average background signal of all images being compared and setting the lower and upper limits of the colormaps to be the same, where the lower limit was equal to a constant A times the average background and the upper limit was equal to a constant $\mathrm{B}$ times the maximum pixel intensity out of all images being compared; images were then converted to $\mathrm{dB}$. For xAM and pre-minus-post-collapse xAM images of bacterial patches, $\mathrm{A}$ was set to 1 and $\mathrm{B}$ was set to 0.5 .

\section{In vitro ultrasound imaging of bacteria expressing ARGs suspended in} agarose phantoms

To create phantoms for ultrasound imaging of bacteria from liquid cultures or suspended in PBS from patches on solid media, wells were cast with a custom 3D-printed mold using $1 \%(\mathrm{w} / \mathrm{v})$ agarose in PBS, which was degassed by incubating at $65^{\circ} \mathrm{C}$ for at least 16 hours. Cultures or cell suspensions to be analyzed were diluted in ice-cold PBS to 2x the final desired cell concentration (calculated from the measured $\mathrm{OD}_{600}$ ), incubated at $42^{\circ} \mathrm{C}$ for one minute, and mixed $1: 1$ with $1 \%$ $(\mathrm{w} / \mathrm{v})$ agarose in $\mathrm{PBS}$ at $42^{\circ} \mathrm{C}$ for a final concentration of $1 \mathrm{x}$. This mixture was then loaded into the wells in duplicate and allowed to solidify. Care was taken not to introduce bubbles during this process. The phantoms were submerged in PBS, and ultrasound images were acquired using a Verasonics Vantage programmable ultrasound scanning system and an L22-14v 128-element linear array transducer with a center frequency of $18.5 \mathrm{MHz}$ with $67 \%-6-\mathrm{dB}$ bandwidth, an element pitch of $100 \mu \mathrm{m}$, an elevation focus of $8 \mathrm{~mm}$, and an elevation aperture of $1.5 \mathrm{~mm}$. The transducer was attached to a custommade manual translation stage to move between samples. Bmode and xAM images were acquired using the same parameters as described previously: 43 the frequency and transmit focus were set to $15.625 \mathrm{MHz}$ and $5 \mathrm{~mm}$, respectively, and each image was an average of 50 accumulations. B-mode imaging was performed with a conventional 128-ray-lines protocol, where each ray line was a single pulse transmitted with an aperture of 40 elements. xAM imaging was performed using a custom sequence detailed previously ${ }^{14}$ with an angle of $19.5^{\circ}$ and an aperture of 65 elements. The transmitted pressure at the focus was calibrated using a Fibre-Optic Hydrophone (Precision Acoustics), and the peak positive pressure was used 
as the "acoustic pressure" in Fig. 3. BURST images were acquired as a series of pAM images as described previously, ${ }^{7}$ except the focus was set to $6 \mathrm{~mm}$, and the acoustic pressure was set to $0.15 \mathrm{MPa}(1.6 \mathrm{~V})$ for the first 10 frames and $3.0 \mathrm{MPa}$ $(25 \mathrm{~V})$ for the last 46 frames.

To measure the xAM signal at varying acoustic pressures, an automated voltage ramp imaging script was used to acquire an xAM image at each voltage step $(0.5 \mathrm{~V}$ increments from 2 to $25 \mathrm{~V}$ ), immediately followed by a B-mode acquisition at a constant voltage of $1.6 \mathrm{~V}(0.15 \mathrm{MPa})$ before another xAM acquisition at the next voltage step; the voltage was held constant for 10 seconds at each step before the image was saved. To measure the xAM and B-mode signals over time at various acoustic pressures, another script was used to automatically save an xAM or B-mode image every second while the voltage was automatically increased by $2 \mathrm{~V}$ approximately every 70 seconds. Each frame consisted of 64 ray lines, which took $180 \mu$ s each to acquire, giving a pulse repetition rate of $86.8 \mathrm{~Hz}$. Based on these results, all subsequent in vitro $\mathrm{xAM}$ images of $\mathrm{bARG}_{\mathrm{Ser}}$-expressing $\mathrm{EcN}$ were acquired at $18 \mathrm{~V}(1.29 \mathrm{MPa})$.

$\mathrm{xAM}$ and $\mathrm{B}$-mode image processing and analysis were performed as described above, except that custom beamforming scripts were applied off-line to reconstruct images from the saved RF data for each sample, no 3D reconstruction was performed as images captured at single locations, circular ROIs were drawn around sample and background regions (taking care to avoid bubbles) to calculate SBRs, and values of $\mathrm{A}=1.4$ and $\mathrm{B}=0.5$ were used to normalize images for display. BURST images were reconstructed using the signal template unmixing algorithm as described previously 20 ; as above, circular ROIs were then drawn around sample and background regions to calculate $\mathrm{SBR}$ and values of $\mathrm{A}=3$ and $\mathrm{B}=1$ were used to normalize images for display.

\section{Microscopy of bacteria}

For TEM imaging, cells expressing GVs were diluted to $\mathrm{OD}_{600}$ $\sim 1$ in $10 \mathrm{mM}$ HEPES (pH 7.5) or culture media. $3 \mu \mathrm{L}$ of the sample was applied to a freshly glow-discharged (Pelco EasiGlow, $15 \mathrm{~mA}, 1 \mathrm{~min}$ ) Formvar/carbon-coated, 200 mesh copper grid (Ted Pella) for $1 \mathrm{~min}$ before being reduced to a thin film by blotting. Grids with cells were washed three times in $10 \mathrm{mM}$ HEPES ( $\mathrm{pH}$ 7.5), blotted, air-dried, and imaged without the stain. Image acquisition was performed using a Tecnai T12 (FEI, now Thermo Fisher Scientific) electron microscope operated at $120 \mathrm{kV}$, equipped with a Gatan Ultrascan 2k X 2k CCD.

For phase contrast microcopy (PCM) imaging, cells expressing GVs were scraped off from plates and re-suspended in PBS at an $\mathrm{OD}_{600}$ of 1-2. Suspensions were transferred to glass slides and PCM images were acquired using a Zeiss Axiocam microscope with a $40 \mathrm{X} \mathrm{Ph} 2$ objective.

\section{In vivo bacterial $A R G$ expression and ultrasound imaging}

All in vivo experiments were performed under a protocol approved by the Institutional Animal Care and Use of Committee (IACUC) of the California Institute of Technology. For experiments involving tumor colonization with $\mathrm{EcN}$,
MC26 cells were grown in DMEM media in T225 flasks. After trypsinization and resuspension in PBS $+0.1 \mathrm{mg} / \mathrm{mL}$ DNAseI, $5 \times 10^{6}$ MC26 cells were injected subcutaneously into the right flank of 6-8-week-old female Balb/cJ mice. Tumors were allowed to grow for 14 days (reaching sizes of 200-300 $\mathrm{mm}^{3}$ ) before injecting $10^{8} \mathrm{EcN}$ cells suspended in PBS via the lateral tail vein. The day before injection of $\mathrm{EcN}$, Ibuprofen was added to the drinking water at $0.2 \mathrm{mg} / \mathrm{mL}$ to ameliorate side effects of $\mathrm{EcN}$ injections. To prepare the $\mathrm{EcN}$ for injection, the appropriate glycerol stocks were used to inoculate $2 \mathrm{~mL} \mathrm{LB}+$ $1 \%(\mathrm{w} / \mathrm{v})$ glucose $+25 \mathrm{ug} / \mathrm{mL}$ chloramphenicol which was incubated at $37^{\circ} \mathrm{C}$ and $250 \mathrm{rpm}$ overnight. The overnight culture $(500 \mu \mathrm{L})$ was used to inoculate $50 \mathrm{~mL} \mathrm{LB}+0.4 \%(\mathrm{w} / \mathrm{v})$ glucose $+25 \mu \mathrm{g} / \mathrm{mL}$ chloramphenicol in $250 \mathrm{~mL}$ baffled flasks, which was grown at $37^{\circ} \mathrm{C}$ and $250 \mathrm{rpm}$ until reaching an $\mathrm{OD}_{600}$ of 0.3 - 0.6. This culture was pelleted, washed 4 times with PBS, resuspended in PBS at an $\mathrm{OD}_{600}$ of 0.625 , and used for injection. Three days after injection of $\mathrm{EcN}$, mice were injected intraperitoneally with $120 \mathrm{mg} \mathrm{L}$-arabinose to induce the EcN. Starting 24 hours after induction, ultrasound images of tumors were acquired as described below. After imaging, 3.0 MPa acoustic pressure was applied throughout the tumor to collapse GVs, and mice were injected again intraperitoneally with $120 \mathrm{mg}$ L-arabinose. The next day, mice were imaged again with ultrasound for re-expression of GVs. The following day, all mice were euthanized and tumors were excised, homogenized, serially diluted, and plated on selective media (LB-agar $+25 \mu \mathrm{g} / \mathrm{mL}$ chloramphenicol) as well as on induction plates (LB-agar $+25 \mu \mathrm{g} / \mathrm{mL}$ chloramphenicol + $0.4 \%(\mathrm{w} / \mathrm{v})$ glucose $+0.1 \%(\mathrm{w} / \mathrm{v})$ L-arabinose). Colonies on plates with chloramphenicol were manually counted to quantify the levels of colonization, and colonies on induction plates were screened for a non-opaque mutant phenotype.

For ultrasound imaging, mice were anesthetized with $2 \%$ isoflurane and maintained at $37^{\circ} \mathrm{C}$ using a heating pad. Images were acquired using the L22-14v transducer attached to a manual translation stage described above. Any hair on or around the tumors was removed with Nair, and Aquasonic 100 ultrasound transmission gel was used to couple the transducer to the skin. B-mode and parabolic AM (pAM) images were first acquired using a custom 128 ray line script. Each image was formed from 96 focused beam ray lines, each with a 32element aperture and $6 \mathrm{~mm}$ focus. The transmit waveform was set to a voltage of $1.6 \mathrm{~V}$ in $\mathrm{B}$-mode or $8 \mathrm{~V}$ in pAM and a frequency of $15.625 \mathrm{MHz}$, with 1 waveform cycle and $67 \%$ intra-pulse duty. In B-mode, each ray line was a single transmit with all 32 elements, and in pAM each ray line consisted of one transmit with all 32 elements followed by 2 transmits in which first the odd and then the even-numbered elements are silenced. ${ }^{37}$ Subsequently, xAM images, additional B-mode images, and finally BURST images were acquired at the same location without moving the transducer using the same parameters as described above for the in vitro experiments (e.g. $18 \mathrm{~V}$ for xAM, $1.6 \mathrm{~V}$ for B-mode, and $1.6 \mathrm{~V}$ to $25 \mathrm{~V}$ for BURST). At least two separate locations spaced at least $2 \mathrm{~mm}$ apart in each tumor were imaged with B-mode, pAM, and xAM. Ultrasound images of tumors were quantified as described above where the sample ROIs were drawn around the necrotic 
cores in the tumors and the background ROIs were drawn around regions in the gel above the mouse. Images were normalized and plotted on a $\mathrm{dB}$ scale as described above except the scaling factors were $\mathrm{A}=2.5$ and $\mathrm{B}=1$ for $\mathrm{xAM}$ and $\mathrm{pAM}$ and the corresponding $\mathrm{B}$-mode tumor images, and $\mathrm{A}=10$ and $\mathrm{B}=0.5$ for BURST images.

\section{Histology of tumors colonized by bacteria}

Tumors were colonized with pBAD-bARG $\mathrm{Ser}_{\mathrm{Axe}}$-Txe EcN following the same protocol as described above. The day after inducing GV expression with IP injections of L-arabinose, $\mathrm{xAM}$ and B-mode images of tumors were acquired as described above. Shortly after imaging, mice were euthanized by sedation with isoflurane and cervical dislocation. Tumors were resected, placed in 10\% buffered formalin for 48 hours, and then washed and stored in $70 \%$ ethanol. Tumors were then cut in half along the approximate plane of imaging, placed in tissue cassettes, and sent to the Translational Pathology Core Laboratory at UCLA, which embedded samples in paraffin and performed H\&E staining, immunohistochemistry, and microscopy imaging. Immunohistochemistry was performed using Opal IHC kits (Akoya Biosciences) according to the manufacturer's instructions. Tissue sections were incubated with either polyclonal rabbit anti-E. coli antibody (Virostat; catalogue number 1001) or non-reactive rabbit IgG isotype control antibody as a negative control. All sections were then incubated with an Opal 520 polymer anti-rabbit HRP antibody (Akoya Biosciences) and counterstained with DAPI. Sections were imaged in the appropriate fluorescence or brightfield channels using a high throughput scanning system (Leica Aperio VERSA) with $40 \mu \mathrm{m}$ resolution.

\section{Mammalian plasmid construction}

Monocistronic plasmids were constructed using standard cloning techniques including Gibson assembly and conventional restriction and ligation. Coding sequences for the A. flos-aquae GV genes were codon-optimized and synthesized by Integrated DNA Technologies, and subcloned into a pCMVSport backbone with a CMV promoter as described previously. ${ }^{7}$ gupA-WPRE-hGH polyA was constructed by subcloning gopA between PstI and MluI sites of pCMVSport vector with WPRE-hGH polyA.

Polycistronic $\mathrm{mARG}_{\text {Ana }}$ assembly factor genes gopNJKFGWV were synthesized by Twist Bioscience in a pTwist-CMV vector. Emerald GFP was subcloned in-frame downstream of the gupNFKFGWV ORF via a P2A linker and the entire $p$ N $f K F G W V$-GFP ORF was subcloned into a pCMVSport backbone with WPRE-hGH-poly(A) elements using NEBuilder HiFi DNA Assembly (NEB). grpA-IRESEBFP2-WPRE-hGH polyA was constructed by Gibson assembly of a PCR-amplified IRES-EBFP2 fragment into the XbaI site of gvpA-WPRE-hGH polyA plasmid.

PiggyBac transposon plasmids were constructed by PCR amplifying the region between the start codon of gupNFKFGWV or gopA and the end of the hGH poly(A) from the pCMVSport plasmids. The amplified regions were Gibsonassembled into the PiggyBac transposon backbone (System
Biosciences) with a TRE3G promoter (Takara Bio) for doxycycline-inducible expression.

The lentiviral transfer plasmid with constitutively expressed tetracycline transactivator (pEFla-rtTA-AntaresWPRE) was constructed as follows: pNCS-Antares was obtained from Addgene (\#74279) and P2A was added to the N-terminus of Antares with a primer overhang during PCR. This fragment was subcloned into the lentiviral transfer plasmid pEFla-rtTA-WPRE between rtTA and WPRE inframe with the rtTA ORF using NEBuilder HiFi DNA Assembly.

HEK293T cell culture, transient transfection, in vitro ultrasound imaging of transient expression of GVs suspended in agarose phantoms

HEK293T cells (ATCG, CLR-2316) were cultured in 24-well plates at $37^{\circ} \mathrm{C}, 5 \% \mathrm{CO} 2$ in a humidified incubator in $0.5 \mathrm{ml}$ DMEM (Corning, 10-013-CV) with 10\% FBS (Gibco) and 1x penicillin/streptomycin until about $80 \%$ confluency before transfection as described previously. ${ }^{7}$ Briefly, transient transfection mixtures were created by mixing of around $600 \mathrm{ng}$ of plasmid mixture with polyethyleneimine (PEI-MAX; Polysciences Inc.) at $2.58 \mu \mathrm{g}$ polyethyleneimine per $\mu \mathrm{g}$ of DNA. The mixture was incubated for 12 minutes at room temperature and added drop-wise to HEK293T cells. Media was changed after 12-16 hours and daily thereafter. For gopA titration experiments, pUC19 plasmid DNA was used to keep the total amount of DNA constant.

After three days of expression, cells were dissociated using Trypsin/EDTA, counted using disposable hemocytometers (Bulldog), and centrifuged at 300g for 6 minutes at room temperature. Cells were resuspended with $1 \%$ low-melt agarose (GoldBio) in PBS at $40^{\circ} \mathrm{C}$ at $\sim 30$ million cells $/ \mathrm{mL}$ (Fig. 5b,f), 15 million cells $/ \mathrm{mL}$ (Fig. 5d), or $\sim 7.5$ million cells $/ \mathrm{mL}$ (Fig S16c) before loading into wells of preformed phantoms consisting of $1 \%$ agarose (Bio-Rad) in PBS.

Phantoms were imaged using L22-14v transducer (Verasonics) while submerged in PBS on top of an acoustic absorber pad. For BURST imaging, wells were centered around the $8 \mathrm{~mm}$ natural focus of the transducer and a BURST pulse sequence was applied in pAM acquisition mode as described above, except the focus was set to $8 \mathrm{~mm}$, and the acoustic pressure was set to $0.26 \mathrm{MPa}(1.6 \mathrm{~V})$ for the first 10 frames and $2.11 \mathrm{MPa}(10 \mathrm{~V})$ for the remaining frames. The xAM voltage ramps and B-mode images were acquired concurrently using the same parameters as described above, except the transducer voltage was varied from 4 to $24 \mathrm{~V}$ in steps of $0.5 \mathrm{~V}$ for $\mathrm{xAM}$, and 10 frames, each consisting of 15 accumulations, were acquired per voltage. The well depth and the B-mode transmit focus were set to $5 \mathrm{~mm}$. All image quantification was performed as described above, where the sample ROIs were drawn inside the well and the background ROIs were drawn around an empty region in the agarose phantom for SBR calculation. All images were normalized and plotted on a $\mathrm{dB}$ scale as described above except the scaling factors were $\mathrm{A}=2$ and $\mathrm{B}=0.5$.

MDA-MB-231 genomic integration and FACS 
MDA-MB-231 (ATCG, HTB26) cells were cultured in DMEM (Corning, 10-013-CV) supplemented with 10\% FBS (Gibco) and $1 \mathrm{x}$ penicillin/streptomycin at $37^{\circ} \mathrm{C}$ and $5 \% \mathrm{CO} 2$ in a humidified incubator unless noted otherwise. Cells were lentivirally transduced with pEFla-rtTA-Antares-WPRE and sorted based on strong Antares fluorescence (Ex: 488 nm, Em: 610/20BP + 595LP) using BD FACSAria II. MDA-MB-231rtTA cells were then electroporated in 20ul format using 4DNucleofector using CH-125 protocol in SF buffer (Lonza) with $1 \mu \mathrm{g}$ PiggyBac transposon:transposase plasmid mixture (2:1 PB-gvpA:PB-gvpNV transposons, 285ng PiggyBac transposase). Cells were expanded into TC treated T75 flasks and were induced for 12 hours with $1 \mu \mathrm{g} / \mathrm{mL}$ doxycycline before sorting for triple positive cells (gated for Antares, then Emerald and EBFP2), which were returned to DMEM with TET-free FBS (Takara). The first round of sorting was performed with permissive gates and the enriched population was $\sim 50 \%$ double positive for Emerald and EBFP2 as analyzed with MACSQuant VYB (Miltenyi Biotec). This population was sorted again with stricter gates to $\sim 95 \%$ purity. Cells were expanded in TET-free media and frozen in Recovery Cell Culture Freezing Medium (Gibco) using Mr. Frosty cell freezing container (Nalgene) filled with isopropanol at $-80^{\circ} \mathrm{C}$, and then stored in liquid nitrogen vapor phase until use.

In vitro ultrasound imaging of MDA-MB-231 mARG $G_{\text {Ana }}$ cells suspended in agarose phantoms

For all in vitro experiments, MDA-MB-231-mARG Ana $_{\text {cells }}$ were cultured in DMEM supplemented with $10 \%$ TET-free FBS and penicillin/streptomycin. For xAM imaging of MDAMB-231-mARG Ana $_{\text {a }}$ cells suspended in agarose phantoms, cells were cultured in 24-well plates in $0.5 \mathrm{~mL}$ media. For Fig. 5i, cells were seeded at 7,500 cells per well and induced with 1 $\mu \mathrm{g} / \mathrm{mL}$ doxycycline after an overnight incubation and at subsequent days as indicated (5 replicates per condition), except for the uninduced control which was grown in a $10 \mathrm{~cm}$ dish without doxycycline. Media was changed daily thereafter until cell harvest. Cells were trypsinized with $100 \mu \mathrm{L}$ Trypsin/EDTA for 6 minutes at $37^{\circ} \mathrm{C}$, after which the trypsin was quenched by addition of $900 \mu \mathrm{L}$ media. The cell number was equalized between different days of expression at 140,000 cells and pelleted at $300 \mathrm{~g}$ for 6 minutes. Cells were then resuspended in $20 \mu \mathrm{l}$ \% low-melt agarose (GoldBio) in PBS at $40^{\circ} \mathrm{C}$ and loaded into the wells of preformed $1 \%$ agarose (BioRad) phantoms in PBS. Ultrasound images were acquired with L22-14v 128-element linear array transducer (Verasonics). $\mathrm{xAM}$ voltage ramp and B-mode images were acquired concurrently using the same parameters as described above (the transducer voltage was varied from 4 to $24 \mathrm{~V}$ in steps of $0.5 \mathrm{~V}$ for $\mathrm{xAM}$ and 10 frames, each consisting of 15 accumulations, were acquired per voltage. The B-mode transmit focus was set to $5 \mathrm{~mm}$ ). Images taken at the voltage that produced peak xAM signal $(9 \mathrm{~V}, 0.54 \mathrm{MPa})$ were chosen for quantification. For Fig. 5j, $\mathbf{k}$, cells were seeded at 66,666 cells per well and induced with the indicated doxycycline concentrations after an overnight incubation in TET-free media (4 replicates per doxycycline concentration). Cells were incubated for 4 days with daily media/doxycycline changes.
Cells were harvested as above, and $\sim 420,000$ cells from each condition were loaded per agarose phantom well. xAM and Bmode images were acquired concurrently using the same parameters as described above except the transducer voltage was varied from $6 \mathrm{~V}$ to $10 \mathrm{~V}$ in steps of $0.5 \mathrm{~V}$ for $\mathrm{xAM}$ and 120 frames, each consisting of 15 accumulations, were acquired per voltage ( 75 seconds/voltage). The B-mode transmit focus was set to $6 \mathrm{~mm}$. Images taken at $7.5 \mathrm{~V}(0.42 \mathrm{MPa})$ were chosen for display and quantification in Fig. 5j (doxycycline response). All image quantification was performed as described above where the sample ROIs were drawn inside the well and the background ROIs were drawn around an empty region in the agarose phantom for SBR calculation. All Images were normalized and plotted on a $\mathrm{dB}$ scale as described above except the scaling factors were $\mathrm{A}=2$ and $\mathrm{B}=0.5$.

\section{TEM imaging of GVs expressed in mammalian cells}

For TEM, cells were cultured in 6-well plates in $2 \mathrm{~mL}$ media. $1 \mu \mathrm{g} / \mathrm{mL}$ doxycycline was added to the wells at indicated times with daily media plus doxycycline changes thereafter until harvest. Cells were lysed by adding $400 \mu \mathrm{L}$ of Solulyse-M (Genlantis) supplemented with 25 units/mL Benzonase Nuclease (Novagen) directly to the 6 -well plates and incubating for 1 hour at $4^{\circ} \mathrm{C}$ with agitation. The lysates were then transferred to $1.5 \mathrm{~mL}$ microcentrifuge tubes. Eight hundred $\mu \mathrm{L}$ of $10 \mathrm{mM}$ HEPES pH 7.5 was added to each tube, and lysates were centrifuged overnight at $300 \mathrm{~g}$ and $8^{\circ} \mathrm{C}$. Thirty $\mu \mathrm{L}$ of the supernatant was collected from the surface from the side of the tube facing the center of the centrifuge rotor and transferred to a new tube. Three $\mu \mathrm{L}$ of each sample was loaded onto freshly glow-discharged (Pelco EasiGlow, $15 \mathrm{~mA}, 1 \mathrm{~min}$ ) formvar/carbon 200 mesh grids (Ted Pella) and blotted after 1 minute then air-dried. The unstained grids were imaged on a FEI Tecnai T12 transmission electron microscope equipped with a Gatan Ultrascan CCD.

In vivo ultrasound imaging of $m A R G_{A n a}$ expressing orthotopic tumors, whole animal fluorescence imaging and tumor fluorescence microscopy.

Tumor xenograft experiments were conducted in NSG mice aged 12-weeks and 6 days (The Jackson Laboratory). To implement an orthotopic model of breast cancer, all the mice were female. MDA-MB-231-mARG $\mathrm{Ana}_{\text {an }}$ cells were grown in T225 flasks in DMEM supplemented with 10\% TET-free FBS and penicillin/streptomycin until confluency as described above. Cells were harvested by trypsinization with $6 \mathrm{~mL}$ Trypsin/EDTA for 6 minutes and quenched with fresh media. Cells were washed once in DMEM without antibiotics or FBS before pelleting by centrifugation at $300 \mathrm{~g}$. Cell pellets were resuspended in 1:1 mixture of ice-cold Matrigel (HC, GFR) (Corning 354263) and $\mathrm{PBS}\left(\mathrm{Ca}^{2+}, \mathrm{Mg}^{2+}\right.$-free) at 30 million cells $/ \mathrm{mL}$. Fifty $\mu \mathrm{L}$ Matrigel suspensions were injected bilaterally into the $4^{\text {th }}$ mammary fat pads at 1.5 million cells per tumor via subcutaneous injection. 12 hours after tumor injection and every 12 hours thereafter (except the mornings of ultrasound imaging sessions) test mice were intraperitoneally injected with $150 \mu \mathrm{L}$ of saline containing $150 \mu \mathrm{g}$ of doxycycline for induction of GV expression. Control mice were not injected with doxycycline. 
For ultrasound imaging, mice were depilated around the $4^{\text {th }}$ mammary fat pads using Nair (Aloe Vera) for ultrasound coupling with Aquasonic 100 gel. Mice were anesthetized with $2.5 \%$ isoflurane and maintained at $37^{\circ} \mathrm{C}$ in supine position on a heating pad. The first imaging session (day 4) consisted of 8 induced tumors from 4 mice and 7 uninduced tumors from 4 mice. One of the uninduced mice died during the first imaging session, which resulted in two fewer uninduced control tumors for the remaining imaging sessions.

Ultrasound images were acquired with an L22-14v 128-element linear array transducer. xAM and B-mode images were acquired concurrently using the same parameters as described in the in vitro section above except the transducer voltage was held at constant $7.5 \mathrm{~V}(0.42 \mathrm{MPa})$ for $\mathrm{xAM}$ and 3 frames, each consisting of 15 accumulations, were acquired per section. A motor stage was programed to move $100 \mu \mathrm{m}$ per section for a total of 150 sections per tumor. The B-mode transmit focus was set to $6 \mathrm{~mm}$. Ultrasound images of tumors were quantified as described above where the sample ROIs were drawn around the tumors and the background ROIs were drawn around regions in the gel above the mouse. Images were normalized and plotted on a $\mathrm{dB}$ scale as described above except the scaling factors were $\mathrm{A}=2$ and $\mathrm{B}=0.5$ for both $\mathrm{xAM}$ and the corresponding B-mode tumor images. The xAM volume quantification was performed by summing all pixel values from all sections in each tomogram between $2 \mathrm{~mm}$ and $10 \mathrm{~mm}$ in depth.

On the last day of ultrasound imaging, mice were anesthetized with $100 \mathrm{mg} / \mathrm{kg}$ ketamine, and $10 \mathrm{mg} / \mathrm{kg}$ xylazine and whole-body imaged in supine position using ChemiDoc MP imaging system with Image Lab software (BIO-RAD). Fluorescence channels were set as follows: blue epi illumination with 530/28 filter for Emerald/GFP and 605/50 filter for Antares/CyOFP1. Images were processed and merged using the FIJI package of ImageJ.

After whole-body fluorescence imaging, mice were euthanized and tumors were resected and placed in $10 \%$ formalin solution for 24 hours at $4^{\circ} \mathrm{C}$, after which they were transferred to PBS. Fixed tumors were embedded in $2 \%$ agarose in PBS and sectioned to $100 \mu \mathrm{m}$ slices using a vibratome. Sections were stained with TO-PRO-3 nucleus stain, mounted using Prolong Glass (Invitrogen) and imaged using a Zeiss LSM 980 confocal microscope with ZEN Blue. Images were processed using the FIJI package of ImageJ. For micrographs of tumors from both induced and uninduced mice, the Emerald channel was capped between 0 and 25497, EBFP2 channel between 0 and 17233 and TO-PRO-3 channel between 5945 and 53136 for display.

Data and code availability

Plasmids will be made available through Addgene upon publication. Ultrasound data acquisition and analysis code will be made available on the Shapiro Lab GitHub. All other materials and data are available from the corresponding author upon reasonable request.

\section{AGKNOWLEDGEMENTS}

The authors would like to thank Dianne Newman for a sample of Streptomyces coelicolor A3(2), and Yunfeng Li, Avinoam BarZion and Hongyi (Richard) Li for help with tissue histology. Electron microscopy was performed in the Beckman Institute Resource Center for Transmission Electron Microscopy at Caltech. Mammalian cell sorting was performed at the Analytical Cytometry Core at City of Hope in Duarte, CA. Confocal microscopy was performed in the Beckman Institute Biological Imaging Center. This research was supported by the National Institutes of Health (R01-EB018975 to M.G.S.) and Pew Charitable Trust. R.C.H. was supported by the Caltech Center for Environmental Microbial Interactions. M.T.B. was supported by an NSF GRFP fellowship. Related research in the Shapiro Laboratory is supported by the David and Lucille Packard Foundation, the Burroughs Wellcome Fund and the Heritage Medical Research Institute.

\section{AUTHOR GONTRIBUTIONS}

R.C.H., M.T.B., M.D., K.W., M.B.S., P.D., Z.J., M.Y.Y., A.F. and R.D. planned and performed experiments. M.T.B. and M.D. performed all in viwo experiments, with help from M.B.S. P.D. and M.D. performed TEM imaging. D.R.M. built the ultrasound plate-scanning setup, and D.R.M. and D.P.S. wrote the associated MATLAB scripts for controlling it. Z.J. and D.P.S. wrote the MATLAB scripts for ultrasound imaging of $\mathrm{EcN}$ in vitro and in vivo. Z.J. performed the calibration of the L22-14v transducer. M.H.A. provided the axe-txe stability cassette, and advised on tumor colonization experiments. R.C.H., M.T.B., M.D., D.P.S., P.D., and Z.J. analyzed data. R.C.H., M.T.B., M.D., and M.G.S. wrote the manuscript with input from all other authors. M.G.S. supervised the research.

\section{COMPETING INTERESTS}

The authors declare no competing financial interests. 


\section{REFERENGES}

1. Piraner, D. I. et al. Going Deeper: Biomolecular Tools for Acoustic and Magnetic Imaging and Control of Cellular Function. Biochemistry 56, 5202-5209 (2017).

2. Maresca, D. et al. Biomolecular Ultrasound and Sonogenetics. Annual Review of Chemical and Biomolecular Engineering 9, 229-252 (2018).

3. Shapiro, M. G. et al. Biogenic Gas Nanostructures as Ultrasonic Molecular Reporters. Nat Nanotechnol 9, 31 1-316 (2014).

4. Walsby, A. E. Gas vesicles. Microbiological Reviewes 58, 94-144 (1994).

5. Pfeifer, F. Distribution, formation and regulation of gas vesicles. Nature Reviewes Microbiology 10, 705-715 (2012).

6. Bourdeau, R. W. et al. Acoustic reporter genes for noninvasive imaging of microbes in mammalian hosts. Nature 553, 86-90 (2018).

7. Farhadi, A., Ho, G. H., Sawyer, D. P., Bourdeau, R. W. \& Shapiro, M. G. Ultrasound imaging of gene expression in mammalian cells. Science 365, 1469-1475 (2019).

8. Sawyer, D. P. et al. Ultrasensitive ultrasound imaging of gene expression with signal unmixing. Nat Methods 18, 945-952 (2021).

9. Chow, B. Y. et al. High-performance genetically targetable optical neural silencing by light-driven proton pumps. Nature 463, 98-102 (2010).

10. Matz, M. V. et al. Fluorescent proteins from nonbioluminescent Anthozoa species. Nature Biotechnology 17, 969973 (1999).

11.Stanton, B. G. et al. Genomic mining of prokaryotic repressors for orthogonal logic gates. Nature Chemical Biology 10, 99-105 (2014).

12. Mak, W. S. et al. Integrative genomic mining for enzyme function to enable engineering of a non-natural biosynthetic pathway. Nature Communications 6, 10005 (2015).

13.Burstein, D. et al. New CRISPR-Cas systems from uncultivated microbes. Nature 542, 237-241 (2017).

14. Maresca, D., Sawyer, D. P., Renaud, G., Lee-Gosselin, A. \& Shapiro, M. G. Nonlinear X-Wave Ultrasound Imaging of Acoustic Biomolecules. Phys. Rev. X 8, 041002 (2018).

15. Tashiro, Y., Monson, R. E., Ramsay, J. P. \& Salmond, G. P. C. Molecular genetic and physical analysis of gas vesicles in buoyant enterobacteria. Environ Microbiol 18, 1264-1276 (2016).

16. Oren, A., Pri-El, N., Shapiro, O. \& Siboni, N. Gas vesicles isolated from Halobacterium cells by lysis in hypotonic solution are structurally weakened. FEMS Microbiology Letters 252, 337-341 (2005).

17.Ramsay, J. P., Williamson, N. R., Spring, D. R. \& Salmond, G. P. C. A quorum-sensing molecule acts as a morphogen controlling gas vesicle organelle biogenesis and adaptive flotation in an enterobacterium. PNAS 108, 14932-14937 (2011).

18.Lee, C. M., Monson, R. E., Adams, R. M. \& Salmond, G. P. G. The LacI-Family Transcription Factor, RbsR, Is a Pleiotropic Regulator of Motility, Virulence, Siderophore and Antibiotic Production, Gas Vesicle Morphogenesis and Flotation in Serratia. Front. Microbiol. 8, (2017).

19.Li, N. \& Cannon, M. C. Gas Vesicle Genes Identified in Bacillus megaterium and Functional Expression in Escherichia coli. f Bacteriol 180, 2450-2458 (1998).

20. Sawyer, D. P. et al. Ultrasensitive ultrasound imaging of gene expression with signal unmixing. Nat Methods 18, 945-952 (2021).

21. Farhadi, A. et al. Genetically Encoded Phase Contrast Agents for Digital Holographic Microscopy. Nano Lett. 20, 8127-8134 (2020).

22.Lu, G. J. et al. Genetically Encodable Contrast Agents for Optical Coherence Tomography. ACS Nano 14, 7823$7831(2020)$.

23.Li, Z. \& Rinas, U. Recombinant protein production associated growth inhibition results mainly from transcription and not from translation. Microb Cell Fact 19, (2020).

24.Borkowski, O., Geroni, F., Stan, G.-B. \& Ellis, T. Overloaded and stressed: whole-cell considerations for bacterial synthetic biology. Current Opinion in Microbiology 33, 123-130 (2016).

25. Sleight, S. C. \& Sauro, H. M. Visualization of Evolutionary Stability Dynamics and Competitive Fitness of Escherichia coli Engineered with Randomized Multigene Circuits. ACS Synth. Biol. 2, 519-528 (2013).

26. Schultz, M. Clinical use of E. coli Nissle 1917 in inflammatory bowel disease. Inflammatory Bowel Diseases 14, 1012-1018 (2008).

27. Stritzker, J. et al. Tumor-specific colonization, tissue distribution, and gene induction by probiotic Escherichia coli Nissle 1917 in live mice. International Fournal of Medical Microbiology 297, 151-162 (2007).

28. Sonnenborn, U. \& Schulze, J. The non-pathogenic Escherichia coli strain Nissle 1917-features of a versatile probiotic. Microbial Ecology in Health and Disease 21, 122-158 (2009). 
29. Gurbatri, G. R. et al. Engineered Probiotics for Local Tumor Delivery of Checkpoint Blockade Nanobodies. Sci Transl Med 12, (2020).

30.Duong, M. T.-Q., Qin, Y., You, S.-H. \& Min, J.-J. Bacteria-cancer interactions: bacteria-based cancer therapy. Experimental \& Molecular Medicine 51, 1-15 (2019).

31.Leventhal, D. S. et al. Immunotherapy with engineered bacteria by targeting the STING pathway for anti-tumor immunity. Nature Communications 11, 2739 (2020).

32.Zhang, Y. et al. E. coli Nissle 1917-Derived Minicells for Targeted Delivery of Chemotherapeutic Drug to Hypoxic Regions for Cancer Therapy. Theranostics 8, 1690-1705 (2018).

33. Grady, R. \& Hayes, F. Axe-Txe, a broad-spectrum proteic toxin-antitoxin system specified by a multidrugresistant, clinical isolate of Enterococcus faecium. Molecular Microbiology 47, 1419-1432 (2003).

34. Ceroni, F. et al. Burden-driven feedback control of gene expression. Nature methods 15, 387 (2018).

35.Donello, J. E., Loeb, J. E. \& Hope, T. J. Woodchuck Hepatitis Virus Contains a Tripartite Posttranscriptional Regulatory Element. 7 Virol 72, 5085-5092 (1998).

36. Lakshmanan, A. et al. Molecular Engineering of Acoustic Protein Nanostructures. ACS Nano 10, 7314-7322 (2016).

37. Maresca, D. et al. Nonlinear ultrasound imaging of nanoscale acoustic biomolecules. Appl Phys Lett 110, (2017).

38.Zhou, X., Vink, M., Klaver, B., Berkhout, B. \& Das, A. T. Optimization of the Tet-On system for regulated gene expression through viral evolution. Gene Ther 13, 1382-1390 (2006).

39.Loew, R., Heinz, N., Hampf, M., Bujard, H. \& Gossen, M. Improved Tet-responsive promoters with minimized background expression. BMC Biotechnol 10, 81 (2010).

40. Chu, J. et al. A bright cyan-excitable orange fluorescent protein facilitates dual-emission microscopy and enhances bioluminescence imaging in vivo. Nat Biotechnol 34, 760-767 (2016).

41. Charbonneau, M. R., Isabella, V. M., Li, N. \& Kurtz, C. B. Developing a new class of engineered live bacterial therapeutics to treat human diseases. Nature Communications 11, 1738 (2020).

42.Jimenez, M., Langer, R. \& Traverso, G. Microbial therapeutics: New opportunities for drug delivery. $\mathcal{J}$ Exp Med 216, 1005-1009 (2019).

43.Lakshmanan, A. et al. Acoustic Biosensors for Ultrasound Imaging of Enzyme Activity. Nat Chem Biol 16, $988-996$ (2020).

44.Hui, C.-Y., Guo, Y., Zhang, W. \& Huang, X.-Q. Rapid monitoring of the target protein expression with a fluorescent signal based on a dicistronic construct in Escherichia coli. AMB Express 8, 81 (2018).

45. Subramanian, S. \& Srienc, F. Quantitative analysis of transient gene expression in mammalian cells using the green fluorescent protein. Fournal of Biotechnology 49, 137-151 (1996).

46. Alnahhas, R. N. et al. Majority sensing in synthetic microbial consortia. Nature Communications 11, 3659 (2020).

47.Hays, S. G., Patrick, W. G., Ziesack, M., Oxman, N. \& Silver, P. A. Better together: engineering and application of microbial symbioses. Current Opinion in Biotechnology 36, 40-49 (2015).

48.Ziesack, M. et al. Engineered Interspecies Amino Acid Cross-Feeding Increases Population Evenness in a Synthetic Bacterial Consortium. mSystems 4, (2019).

49. Wacklin, P., Hoffmann, L. \& Komárek, J. Nomenclatural validation of the genetically revised cyanobacterial genus Dolichospermum (RALFS ex BORNET et FLAHAULT) comb. nova. Fottea 9, 59-64 (2009).

50.Komárek, J. et al. Polyphasic evaluation of Limnoraphis robusta, a water-bloom forming cyanobacterium from Lake Atitlán, Guatemala, with a description of Limnoraphis gen. nov. Fottea 13, 39-52 (2013).

51.Komárek, J. Planktic oscillatorialean cyanoprokaryotes (short review according to combined phenotype and molecular aspects). in Phytoplankton and Equilibrium Concept: The Ecology of Steady-State Assemblages (eds. Naselli-Flores, L., Padisák, J. \& Dokulil, M. T.) 367-382 (Springer Netherlands, 2003). doi:10.1007/978-94-017-2666-5_30.

52. Sievers, F. et al. Fast, scalable generation of high-quality protein multiple sequence alignments using Clustal Omega. Molecular Systems Biology 7, 539 (2011).

53. Larkin, M. A. et al. Clustal W and Clustal X version 2.0. Bioinformatics 23, 2947-2948 (2007).

54.Subramanian, B., Gao, S., Lercher, M. J., Hu, S. \& Chen, W.-H. Evolview v3: a webserver for visualization, annotation, and management of phylogenetic trees. Nucleic Acids Research 47, W270-W275 (2019).

55.Ammar, E. M., Wang, X. \& Rao, C. V. Regulation of metabolism in Escherichia coli during growth on mixtures of the non-glucose sugars: arabinose, lactose, and xylose. Sci Rep 8, 609 (2018). 
bioRxiv preprint doi: https://doi.org/10.1101/2021.04.26.441537; this version posted October 29, 2021. The copyright holder for this preprint (which was not certified by peer review) is the author/funder. All rights reserved. No reuse allowed without permission.

\section{Supplementary Information}

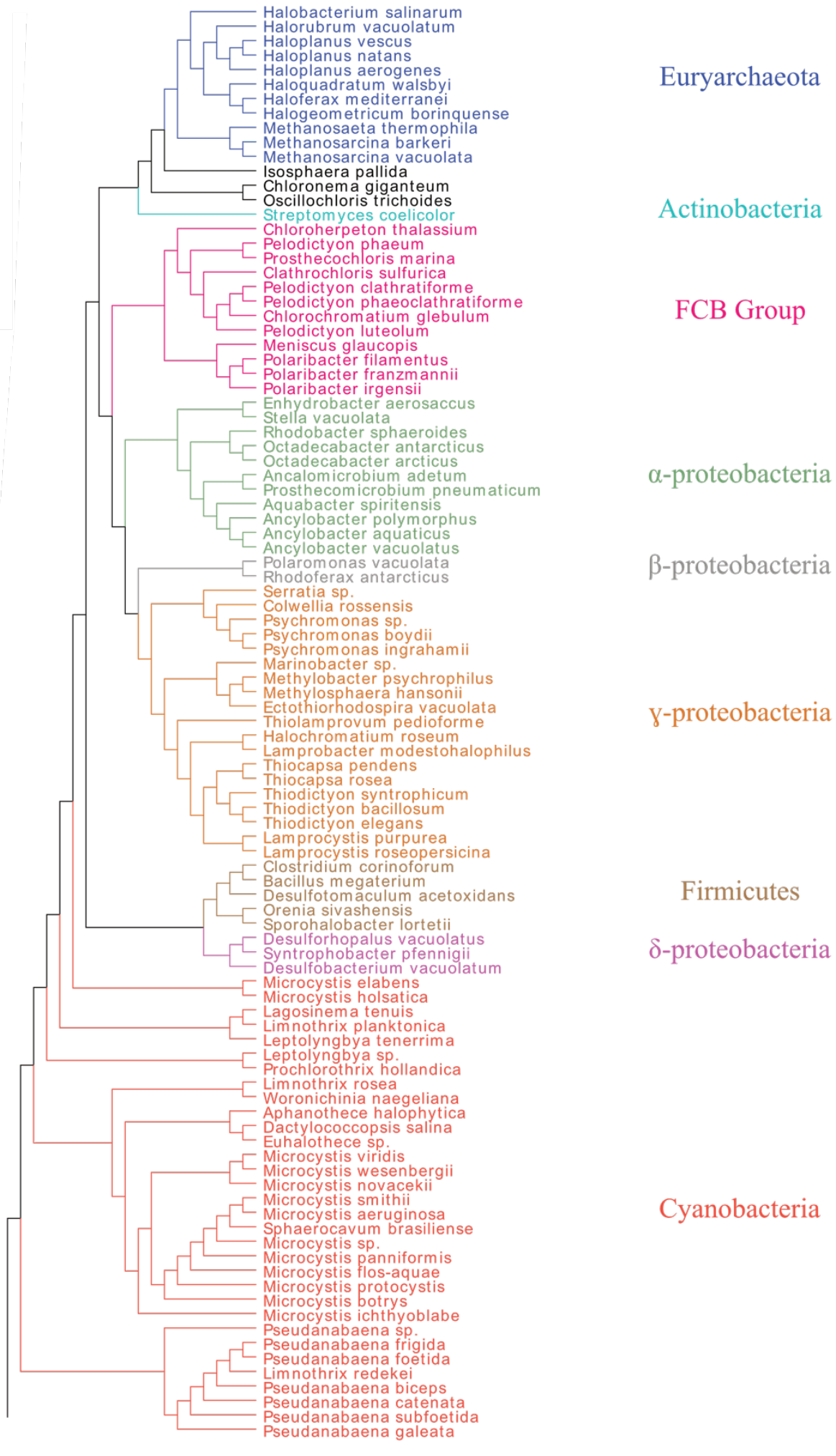


bioRxiv preprint doi: https://doi.org/10.1101/2021.04.26.441537; this version posted October 29, 2021. The copyright holder for this preprint (which was not certified by peer review) is the author/funder. All rights reserved. No reuse allowed without permission.

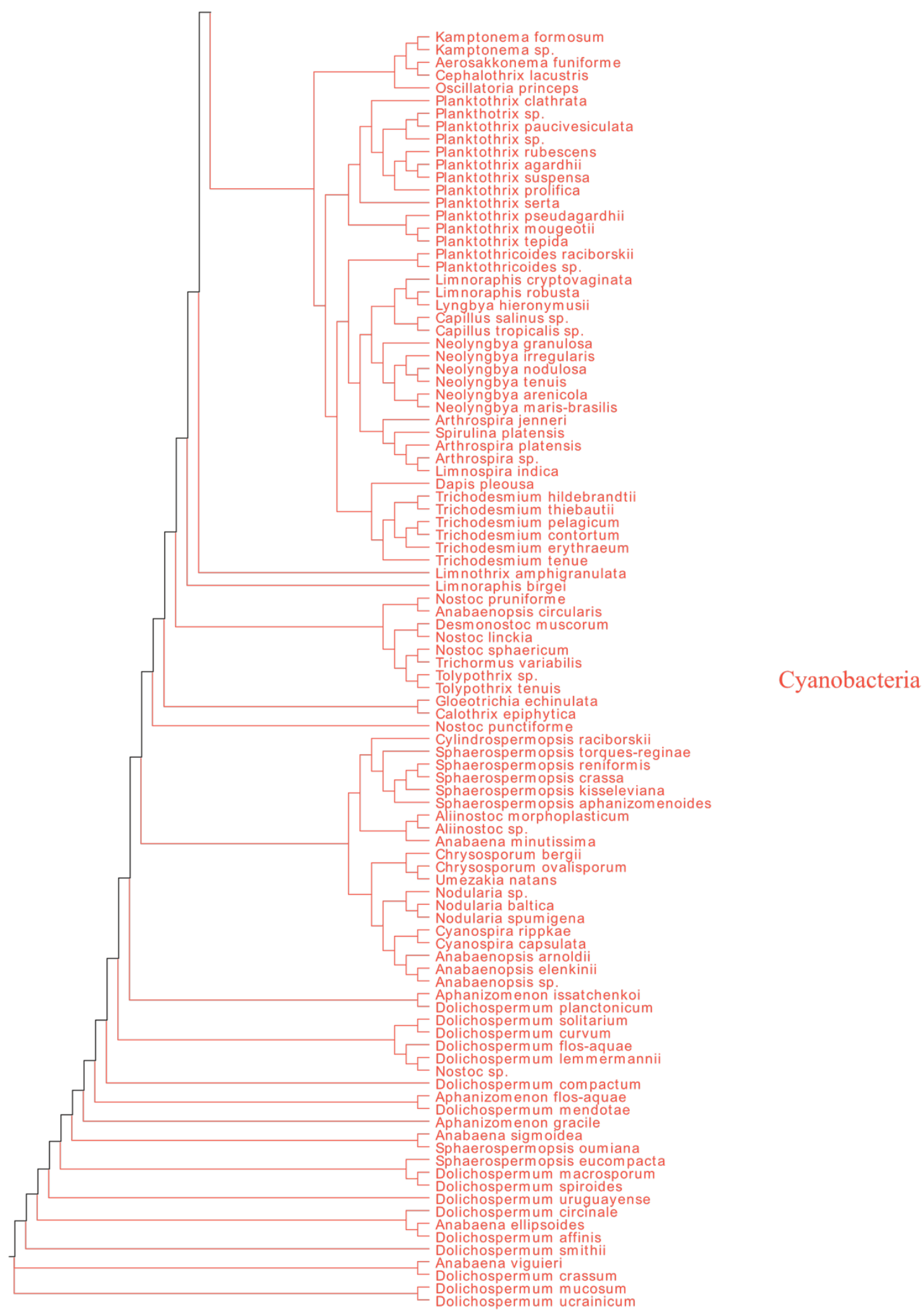

Figure S1 | $16 \mathrm{~S}$ phylogenetic tree of all reported GV-producing organisms. Colors indicate groupings of phylogenetically similar organisms. Organisms from which GV genes were tested in E. coli are shown in Fig. 1a. 


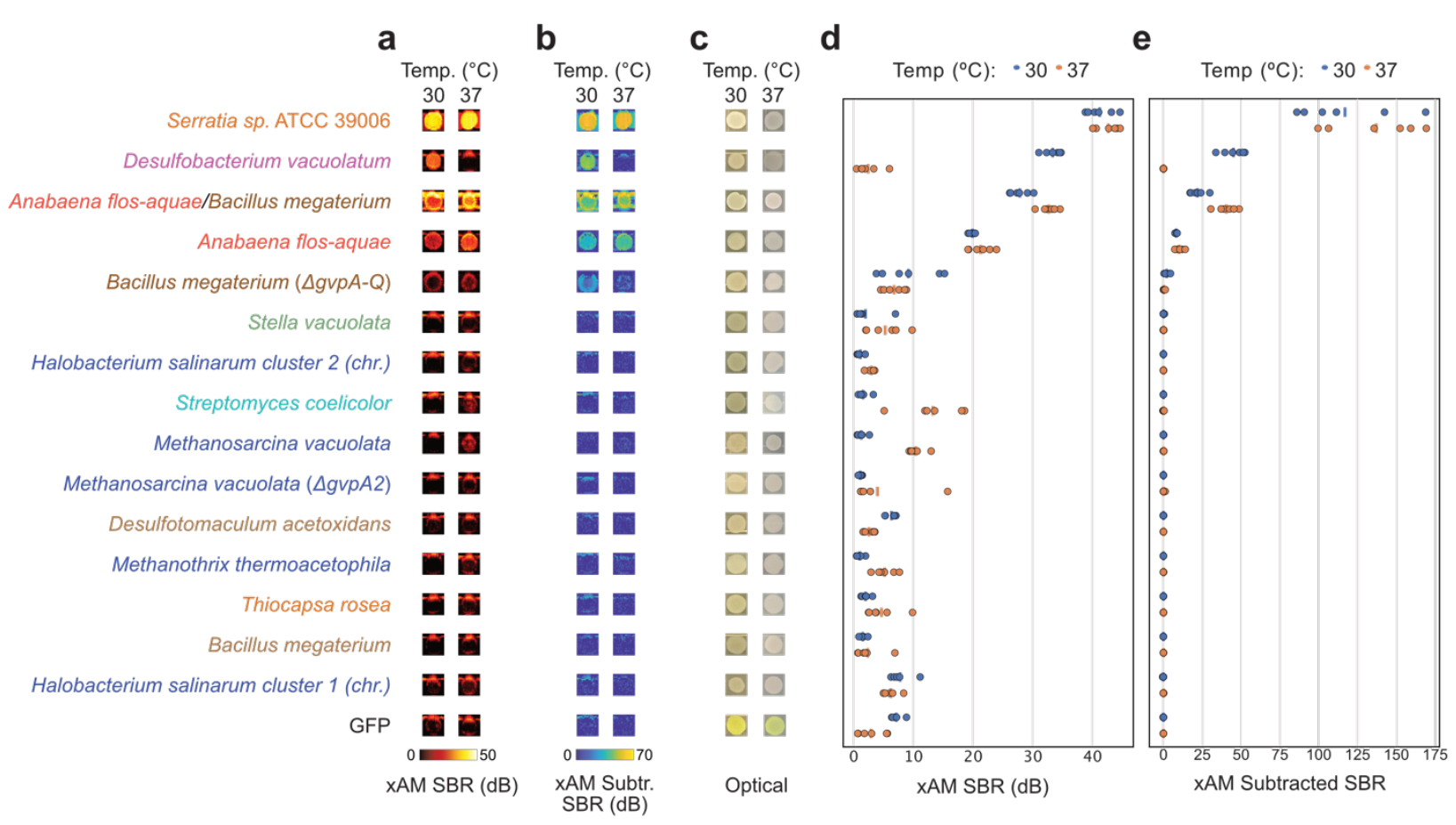

Figure S2 | Additional images and quantification of $\boldsymbol{E}$. coli patches expressing select GV gene clusters. (a-c) xAM images (a), pre-minus-post-collapse xAM images (b), and optical images showing opacity (c) of patches of $E$. coli expressing various GV gene clusters from the organisms listed on the left. (d-e) Quantification of the xAM (d) and pre-minus-post-collapse xAM (e) signals from images in $(a-b)(n=6)$. SBR, signal-to-background ratio. 
a

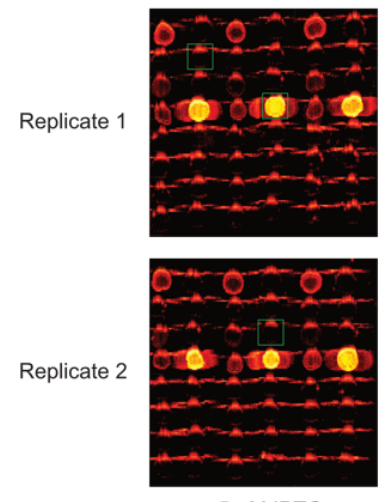

$5 \mu$ M IPTG

b

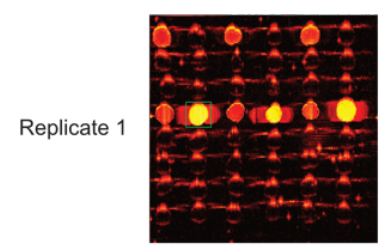

Replicate 2

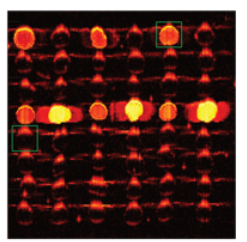

$5 \mu$ M IPTG
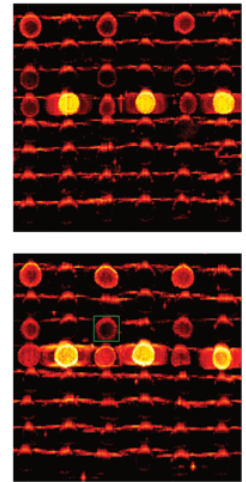

10 MM IPTG
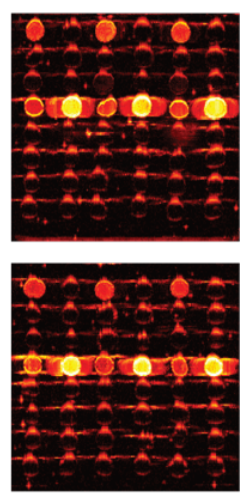

$10 \mu$ M IPTG $30^{\circ} \mathrm{C}$

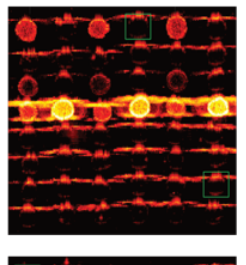

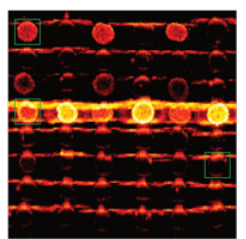

25 M IPTG

$37^{\circ} \mathrm{C}$
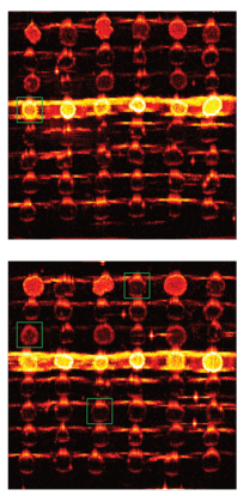

25 M IPTG
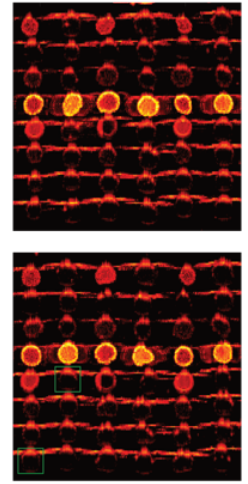

50 M IPTG
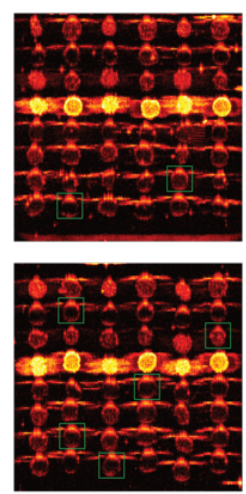

50 M IPTG
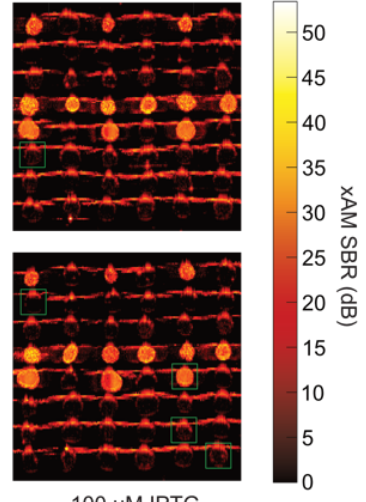

$100 \mu$ M IPTG
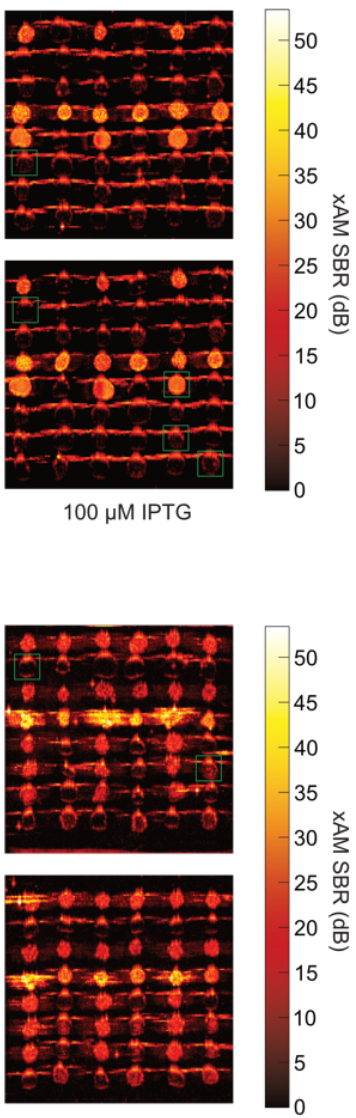

100 MM IPTG
C

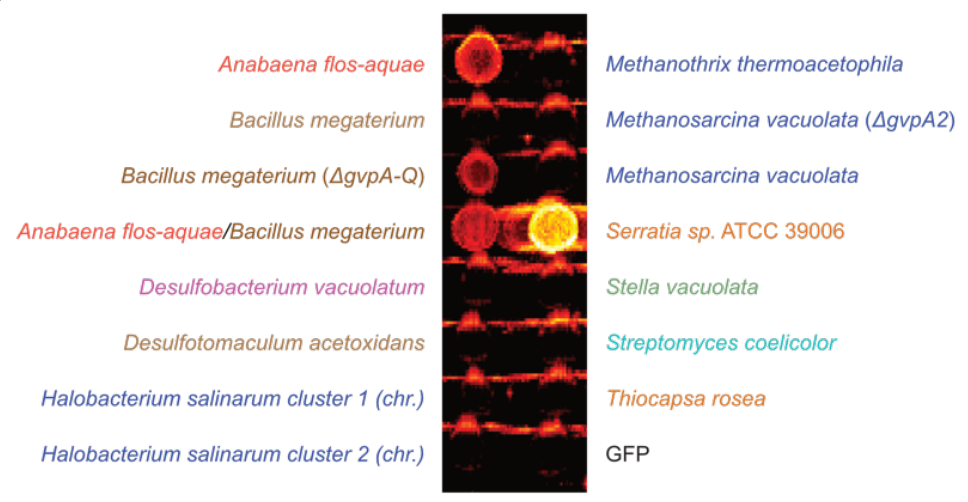

d

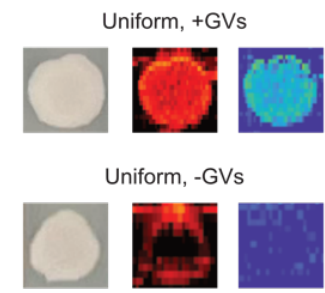

Punctate, +GVs

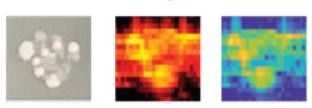

Punctate, -GVs

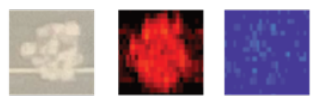

Normal OD, -GVs
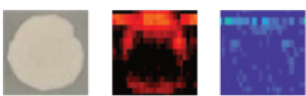

Reduced OD, -GVs

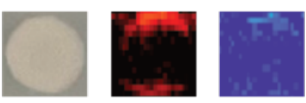


bioRxiv preprint doi: https://doi.org/10.1101/2021.04.26.441537; this version posted October 29, 2021. The copyright holder for this preprint (which was not certified by peer review) is the author/funder. All rights reserved. No reuse allowed without permission.

Figure S3 | Optimization of expression conditions for all tested clusters in BL21(DE3) $\boldsymbol{E}$. coli. (a-b) xAM images of bacterial patches expressing each GV cluster at varying inducer concentrations and temperatures. Green boxes indicate the patches shown in Fig. S2a. The IPTG concentration selected was the one that resulted in the highest xAM pre-minus-post-collapse difference signal $($ Fig. S6 $)$ while not creating toxicity, as determined by whether the patch was uniform or punctate $($ Fig. S5a-b). Some of the IPTG concentrations that led to toxicity also created significant xAM signal, but this signal did not originate from GVs, as indicated by the lack of xAM pre-minus-post-collapse signal difference (Fig. S6). Further, there were some IPTG concentrations for certain genotypes that created significant xAM signal but no xAM pre-minus-post-collapse signal difference, and no visible toxicity (e.g., Streptomyces coelicolor, Thiocapsa rosea, and GFP at $37^{\circ} \mathrm{C}, 100 \mu \mathrm{M}$ IPTG). This discrepancy was likely caused by subtle toxicity that is not apparent in optical images, but altered the texture of the patch enough to be detectable by US. (c) Key for genotypes tested in (a-b), with this pattern repeated in three pairs of columns replicated on each plate. (d) Examples of the effects of toxic genotypes on bacterial patches, and of artifacts that can appear in bacterial patch scan images. Bacteria themselves can produce significant xAM signal (especially when present in extremely high concentrations, as they are in the confluent patches imaged here), which can be seen in the forms of rings around all patches, regardless of GV expression status. Further, expression of toxic proteins (or of large amounts of otherwise non-toxic proteins, such as GFP) can interfere with bacterial growth; in extreme cases this results in significant cell death and a punctate appearance, and in less extreme cases it simply reduces the optical density of patches. GV expression can increase the optical density of patches, but only at high levels of GV expression. Punctate patches produce considerably more xAM signal than uniform ones, even in the absence of GV expression. The xAM pre-minus-postcollapse difference can be used to qualitatively determine if a patch produces $\mathrm{GVs}$, but because collapse is incomplete in some cases, it is not an ideal method for quantitatively comparing genotypes. 
a $30^{\circ} \mathrm{C}$

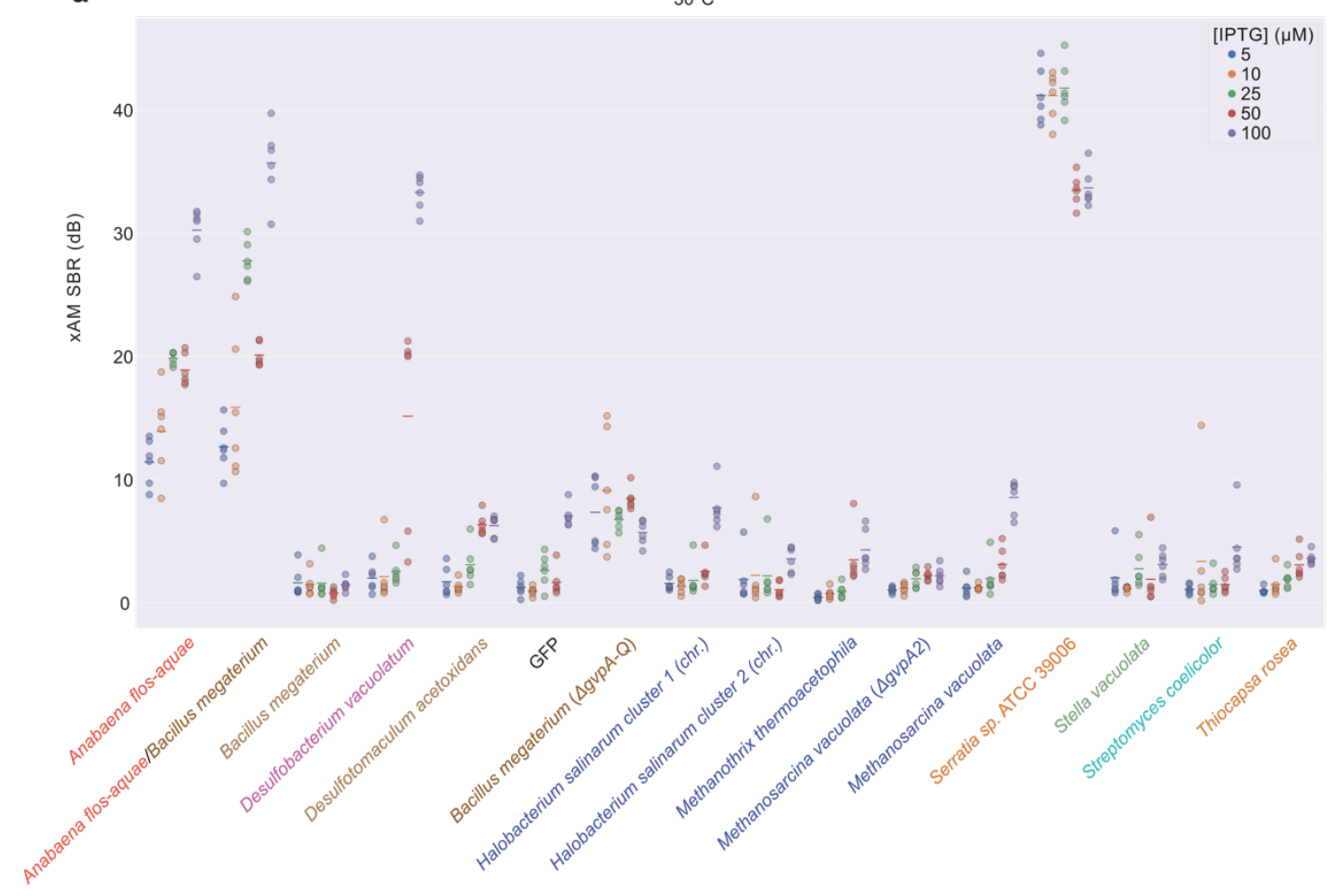

b

$37^{\circ} \mathrm{C}$

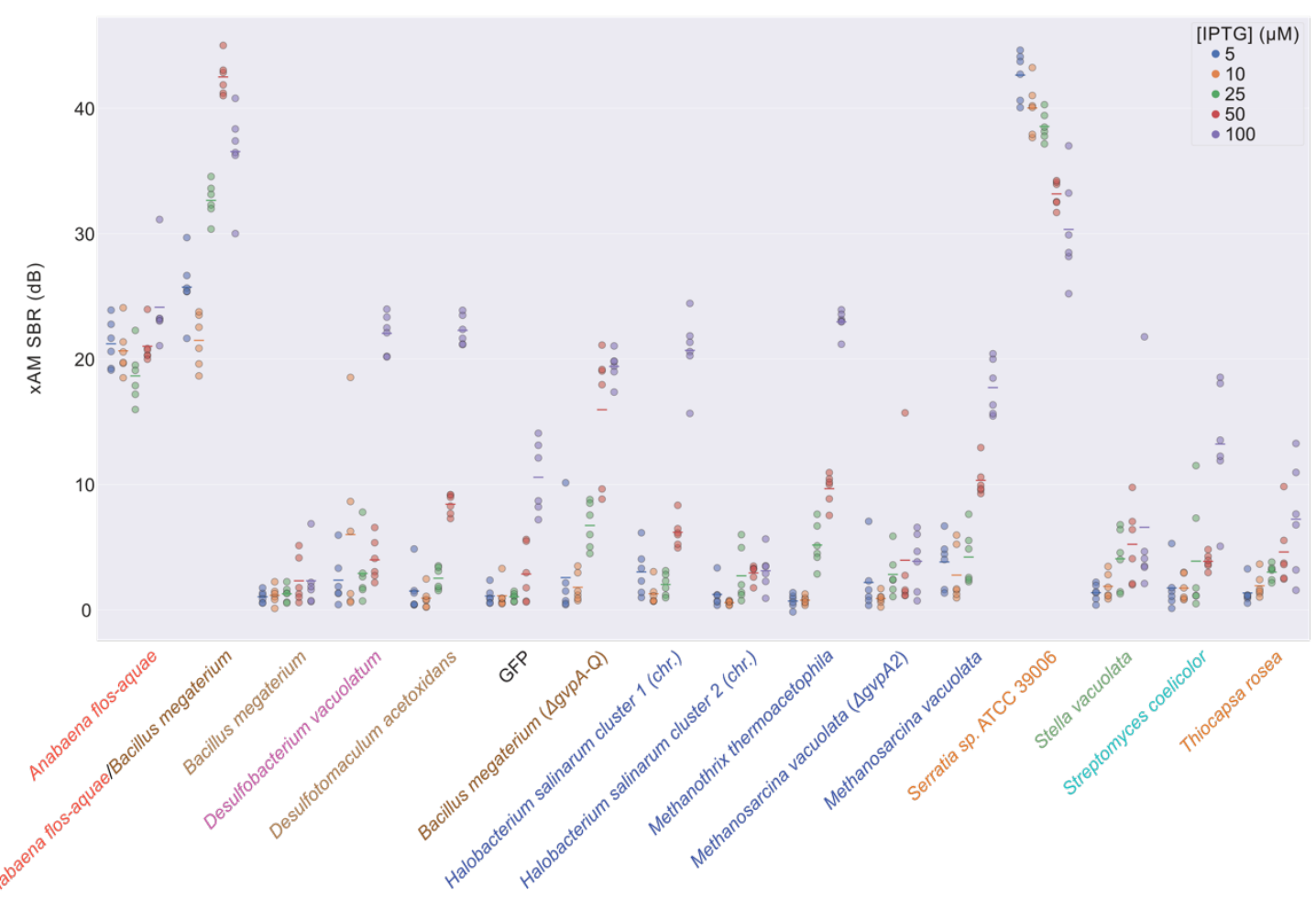

Figure S4 | Quantification of ultrasound signal for all samples shown in Fig. S3a-b. (a-b) xAM SBR of the patches at $30^{\circ} \mathrm{C}\left(\right.$ a) and $37^{\circ} \mathrm{C}($ b) shown in Fig. S3a-b. 
bioRxiv preprint doi: https://doi.org/10.1101/2021.04.26.441537; this version posted October 29, 2021. The copyright holder for this preprint (which was not certified by peer review) is the author/funder. All rights reserved. No reuse allowed without permission.

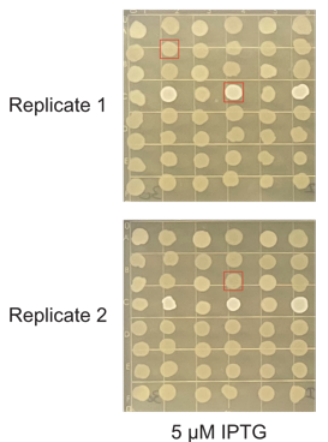

b

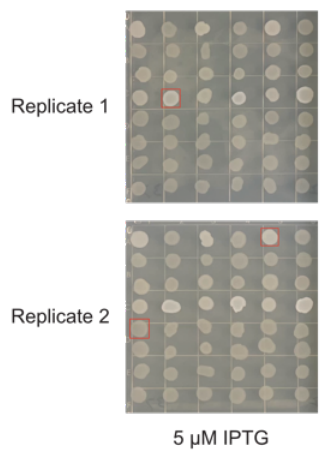

\section{C}

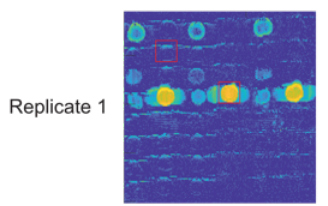

Replicate 2

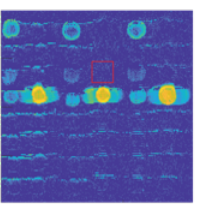

5 MM IPTG

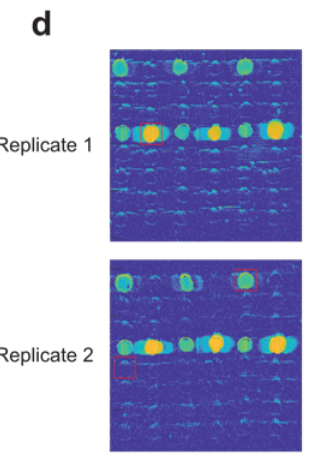

5 HM IPTG

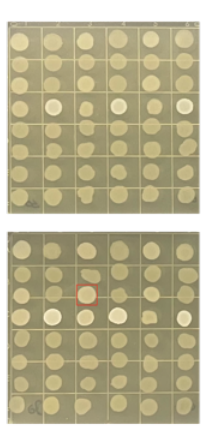

$10 \mu$ M IPTG
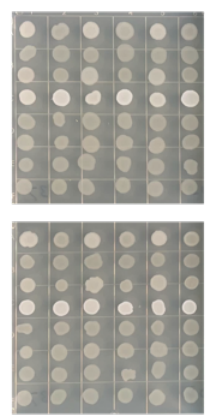

$10 \mu \mathrm{M}$ IPTG
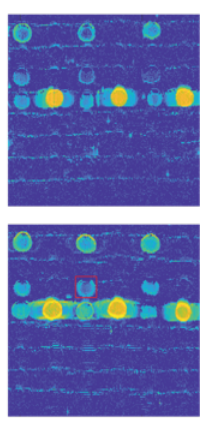

$10 \mu \mathrm{M}$ IPTG
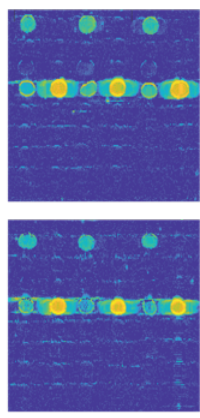

$10 \mu \mathrm{M}$ IPTG $30^{\circ} \mathrm{C}$
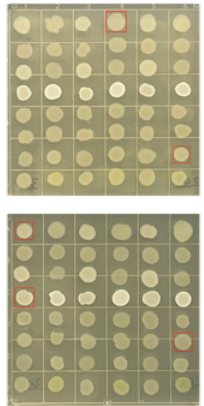

25 मM IPTG

$37^{\circ} \mathrm{C}$
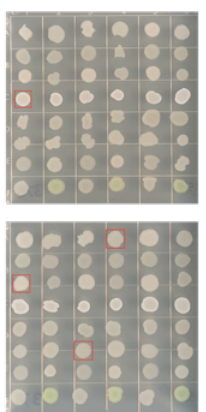

$25 \mu$ M IPTG
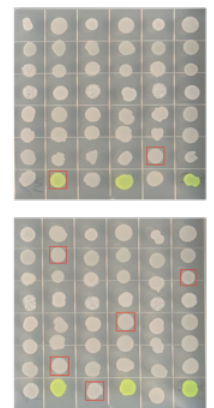

$50 \mu \mathrm{M}$ IPTG
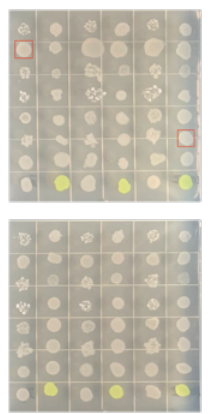

$100 \mu$ M IPTG
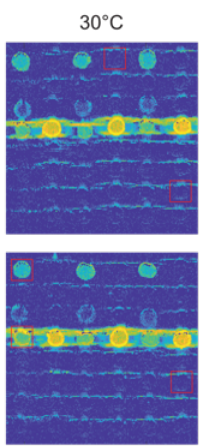

$25 \mu \mathrm{M}$ IPTG
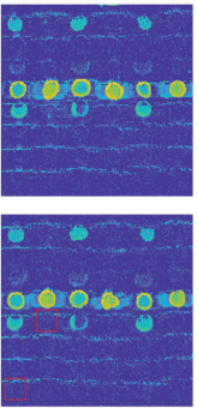

$50 \mu \mathrm{M}$ IPTG
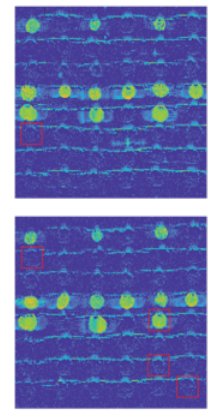

$100 \mu$ M IPTG

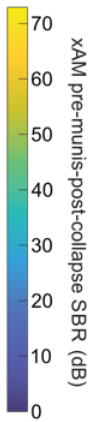

$37^{\circ} \mathrm{C}$
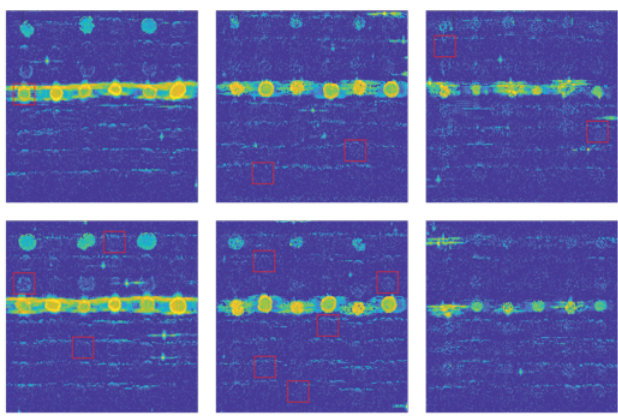

$25 \mu \mathrm{M}$ IPTG

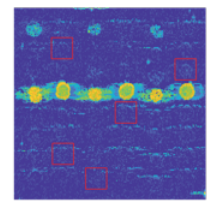

50 M IPTG

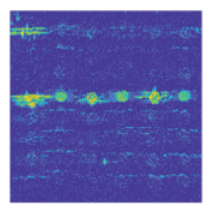

100 HM IPTG

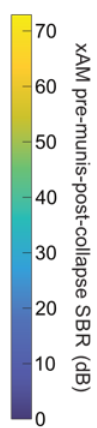

Figure S5 | Optical and xAM pre-minus-post-collapse difference images of all samples shown in Fig. S3. (a-b) Optical images of patches at $30^{\circ} \mathrm{C}\left(\right.$ a) and $37^{\circ} \mathrm{C}$ (b) shown in Fig. S3a-b. (c-d) xAM pre-minus-post-collapse difference patches of samples at $30^{\circ} \mathrm{C}$ (a) and $37 \circ^{\circ} \mathrm{C}$ (b) shown in Fig. S3a-b. Red boxes indicate the patches shown in Fig. S2b-c. 
a

$30^{\circ} \mathrm{C}$

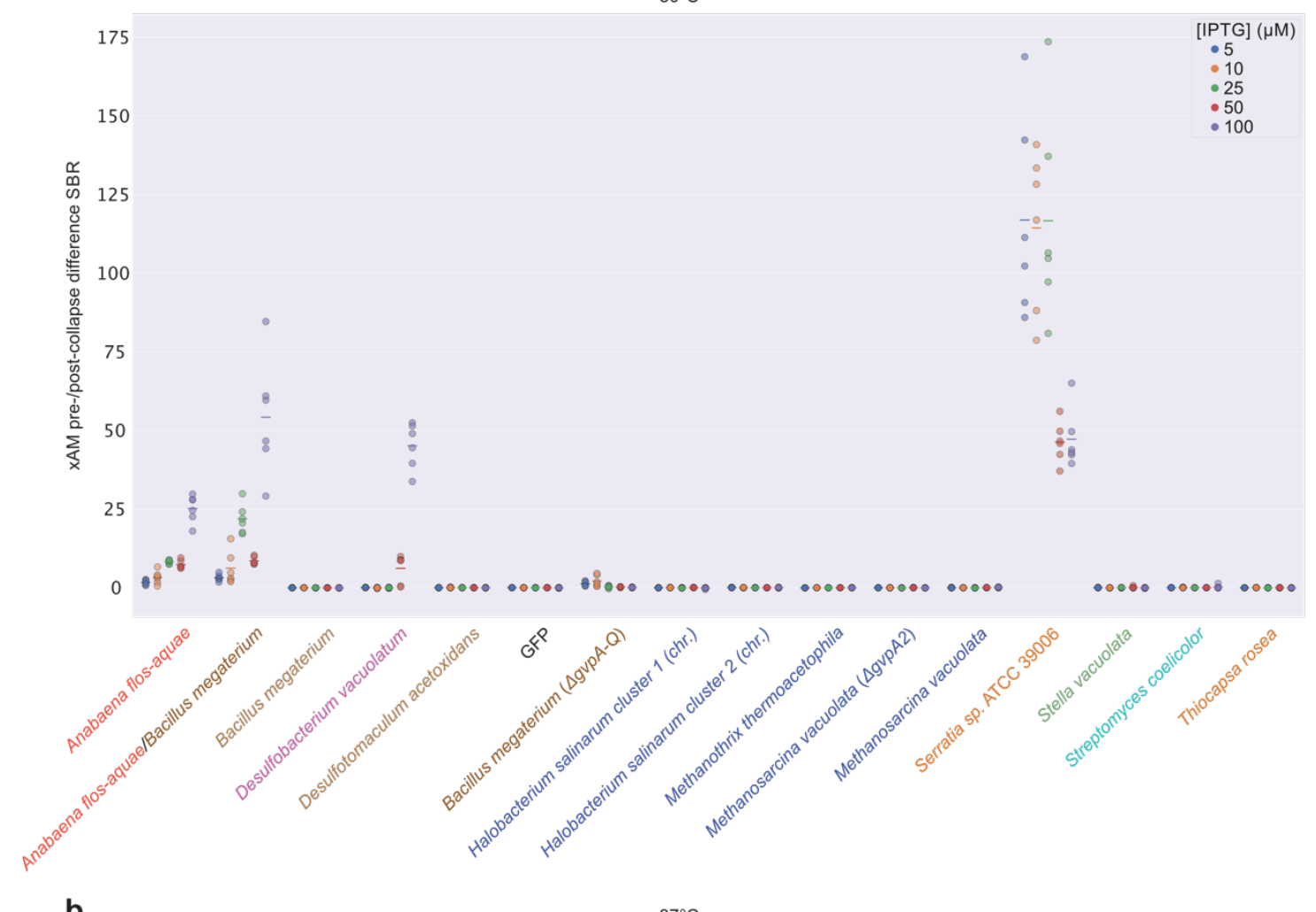

b

$37^{\circ} \mathrm{C}$

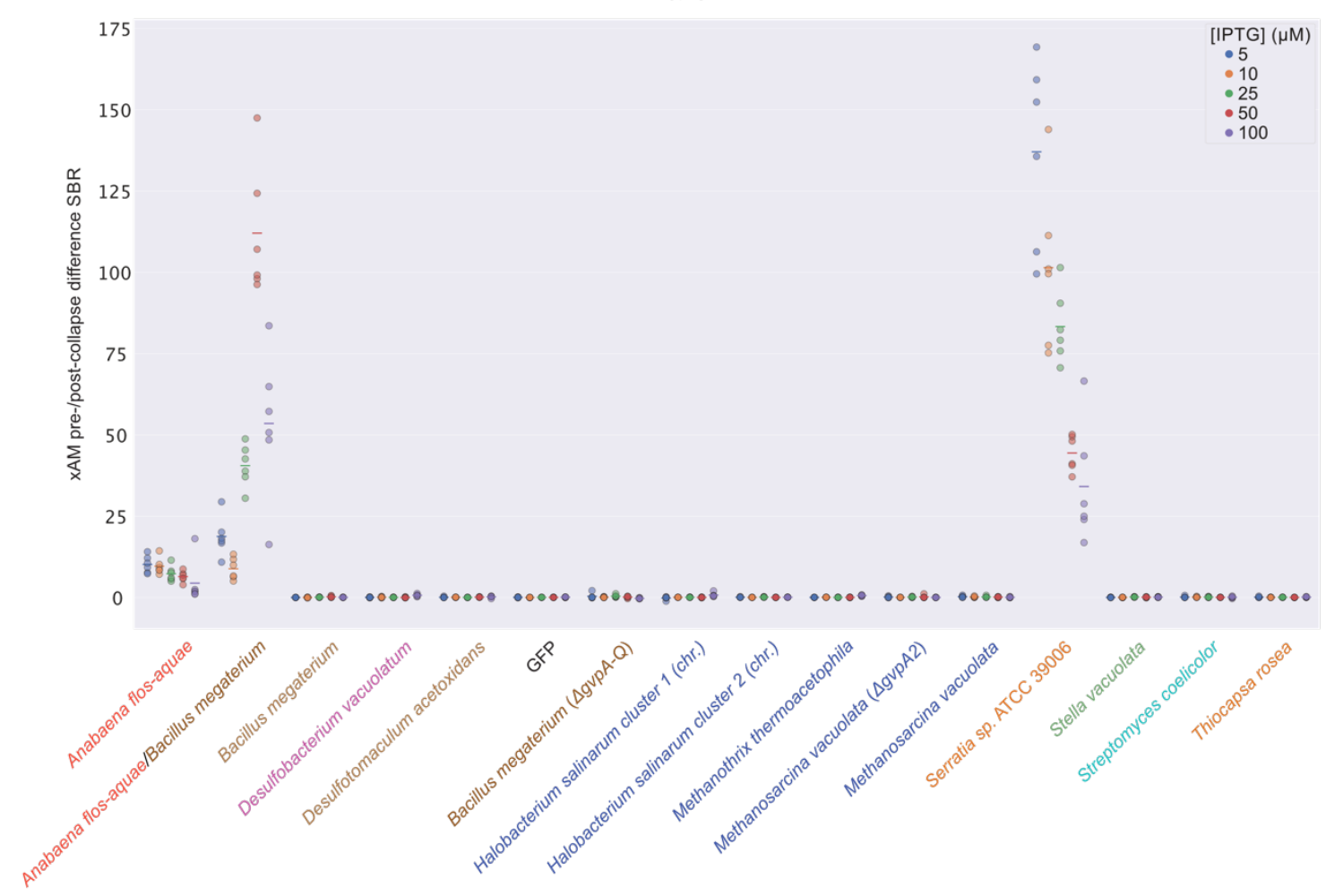

Figure S6 | Quantification of ultrasound signal for samples shown in Fig. S5c-d. (a-b) xAM SBR for the patches at $30^{\circ} \mathrm{C}(\mathrm{a})$ and $37^{\circ} \mathrm{C}(\mathrm{b})$ shown in Fig. S5c-d. 
bioRxiv preprint doi: https://doi.org/10.1101/2021.04.26.441537; this version posted October 29, 2021. The copyright holder for this preprint (which was not certified by peer review) is the author/funder. All rights reserved. No reuse allowed without permission.
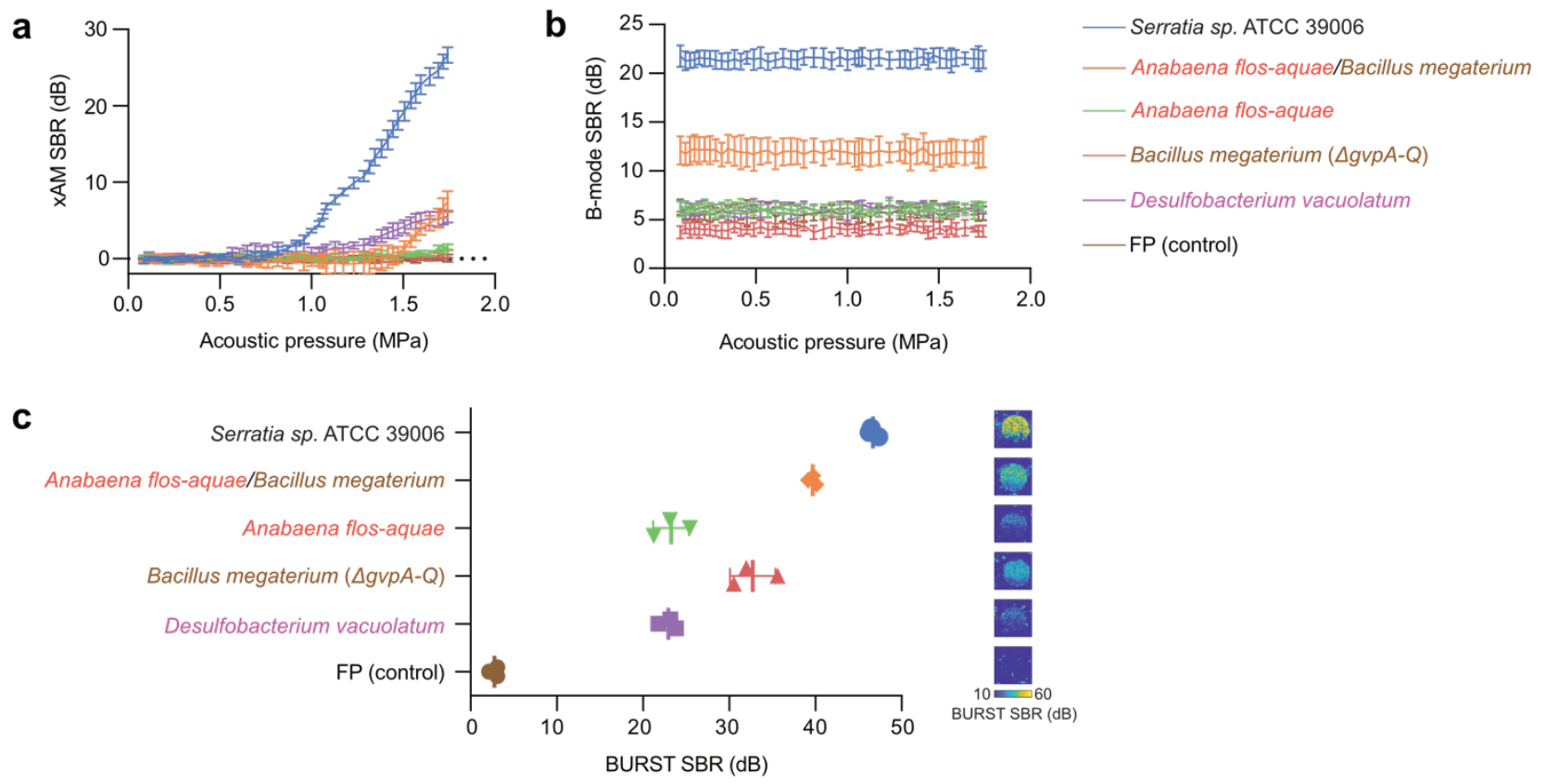

d
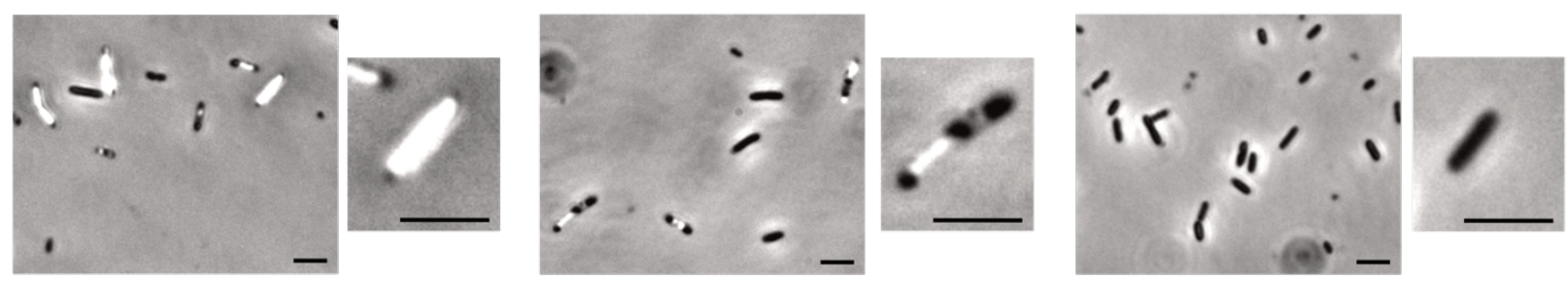

Bacillus megaterium ( $\triangle g v p A-Q)$
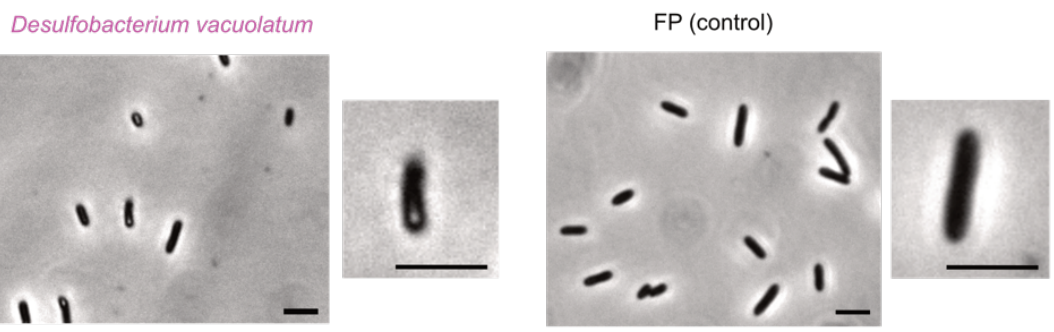

\section{e}
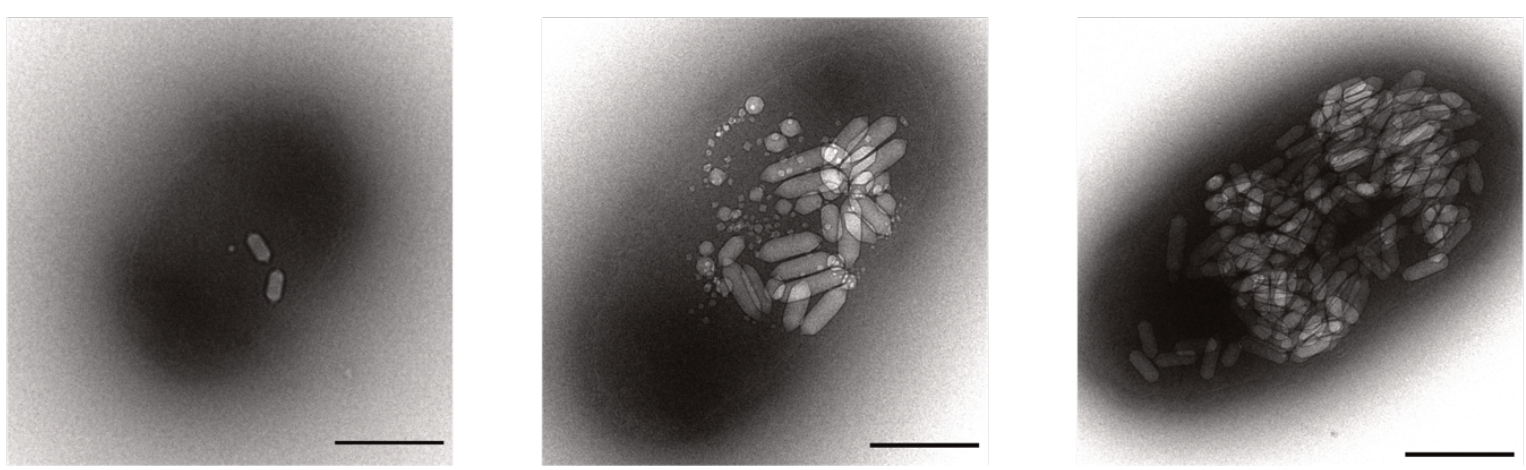
bioRxiv preprint doi: https://doi.org/10.1101/2021.04.26.441537; this version posted October 29, 2021. The copyright holder for this preprint (which was not certified by peer review) is the author/funder. All rights reserved. No reuse allowed without permission.

Figure S7 | Characterization of working GV clusters in BL21(DE3) E. coli. (a-d) xAM signal-to-background ratio (SBR) as a function of acoustic pressure (a), B-mode SBR at a constant pressure of $0.15 \mathrm{MPa}$ after each increase in acoustic pressure in a (b), BURST SBRs and corresponding representative images (c), and representative phase contrast microscopy (PCM) images (d) of the working GV clusters expressed in BL21(DE3) E. coli at $30^{\circ} \mathrm{C}$ on solid media. For ultrasound imaging (a-c), samples were normalized to $5 \times 10^{9}$ cells $/ \mathrm{mL}$ in agarose phantoms. Curves and error bars represent the mean $(\mathrm{n}=3$ biological replicates each with 2 technical replicates) \pm SD. (a-b) have the same legend. GV clusters in cells are visible by PCM for all clusters except for the cluster from Anabaena flos-aquae and the fluorescent protein (FP) control (d). (e) Representative TEM images of BL21(DE3) E. coli cells expressing Serratia GVs at varying levels of expression. Scale bars are $5 \mu \mathrm{m}$ in (d) and $500 \mathrm{~nm}$ in (e). 
a

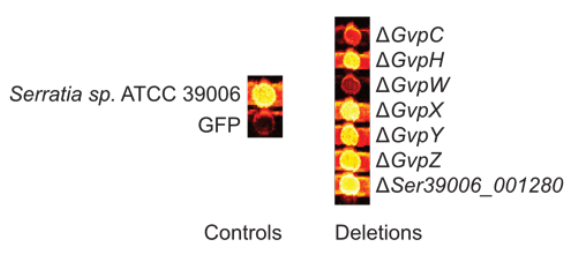

C

e

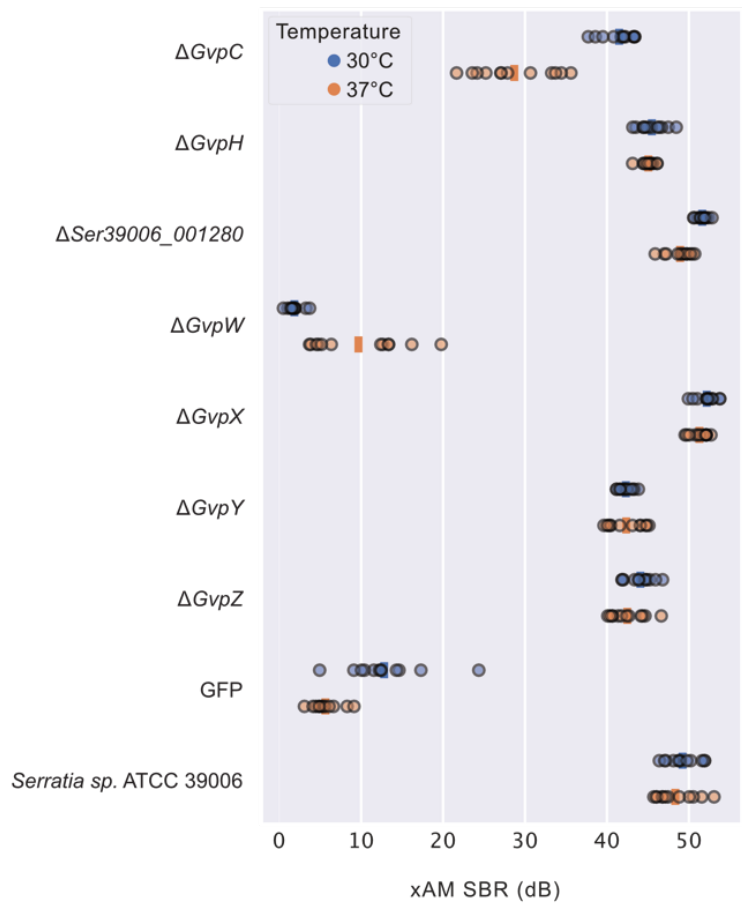

b

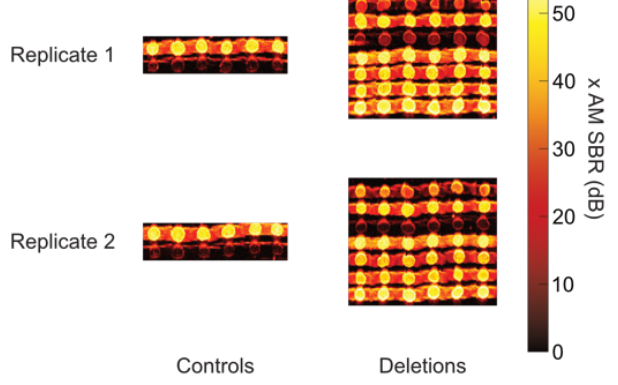

d

Replicate 188000

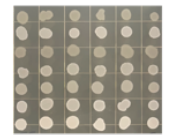

Replicate 2
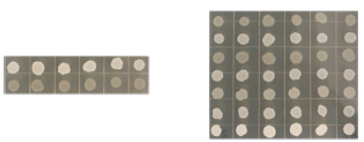

Controls

Deletions

f

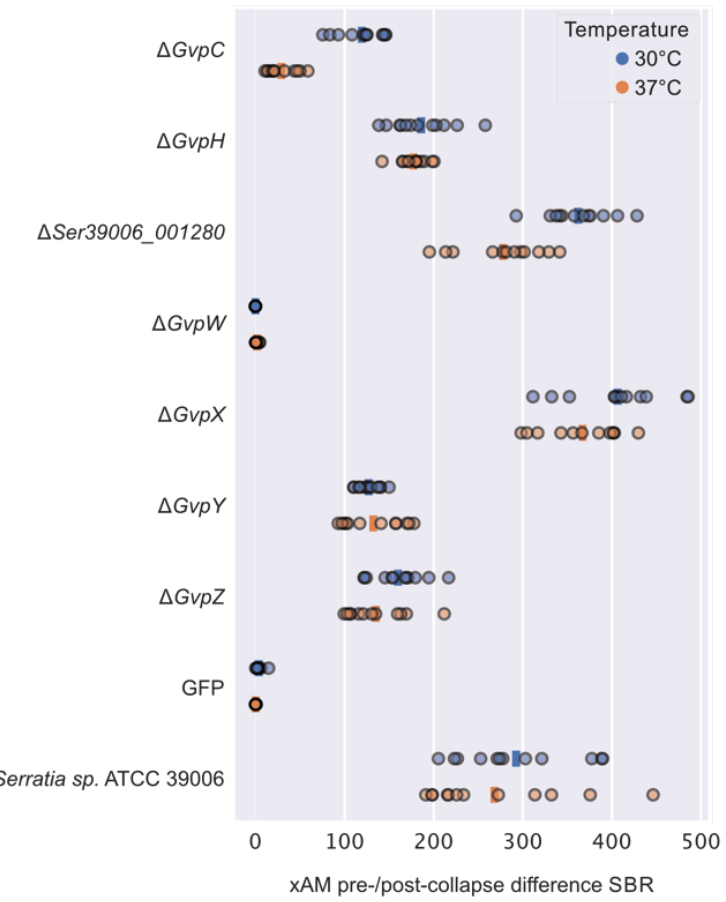

Figure S8 I Effects of single-gene deletions on GV expression by the Serratia cluster. (a) Key for genotypes tested, repeated in 6 replicate columns on each plate. (b-d) xAM images (b), pre-minus-post-collapse xAM images (c), and optical images (d) of bacterial patches expressing single-gene deletions of the Serratia cluster. (e-f) Quantification of the xAM images (e) and preminus-post-collapse xAM images (f) shown in (b-c). 
a
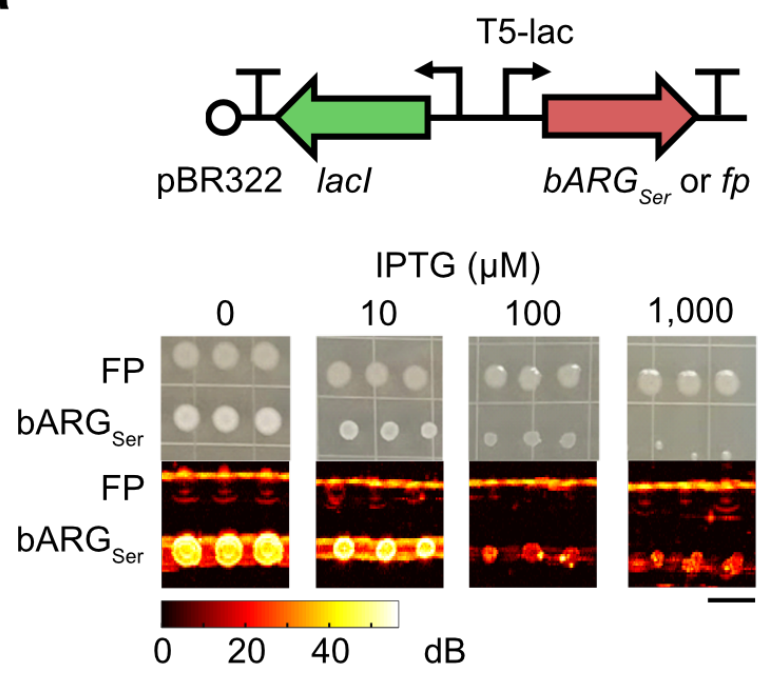

C

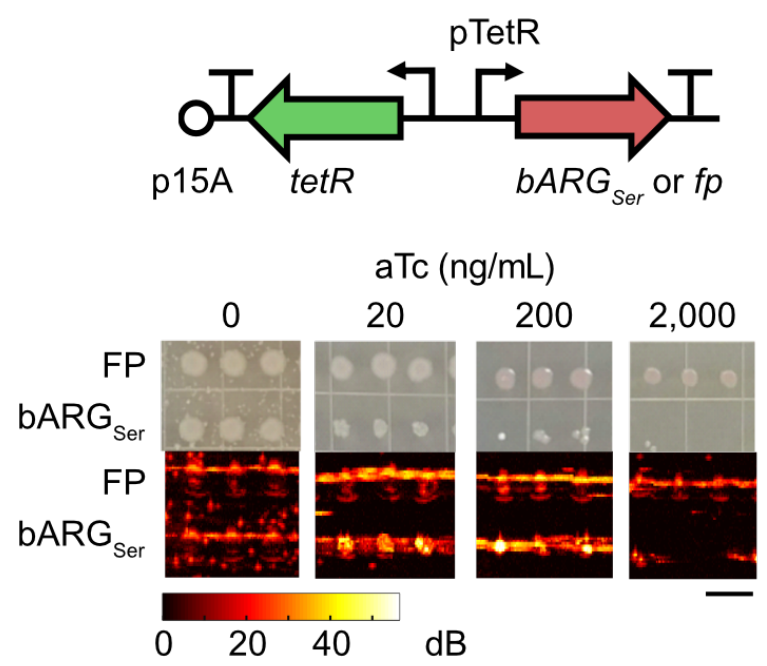

b

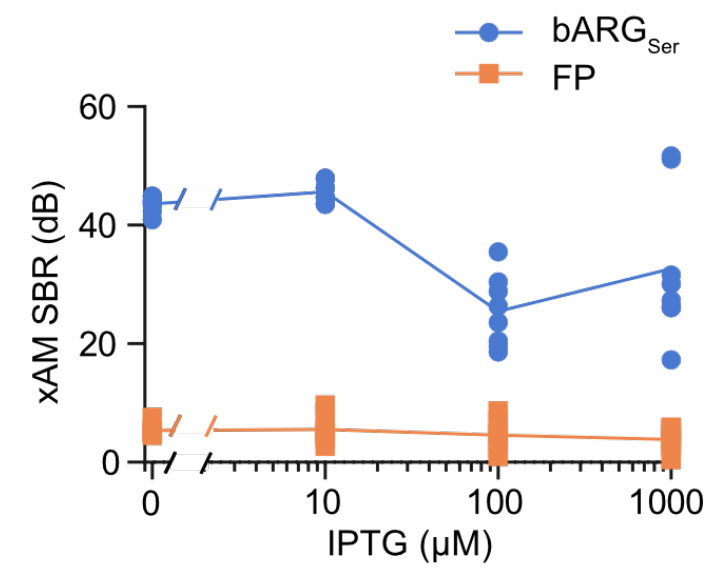

d

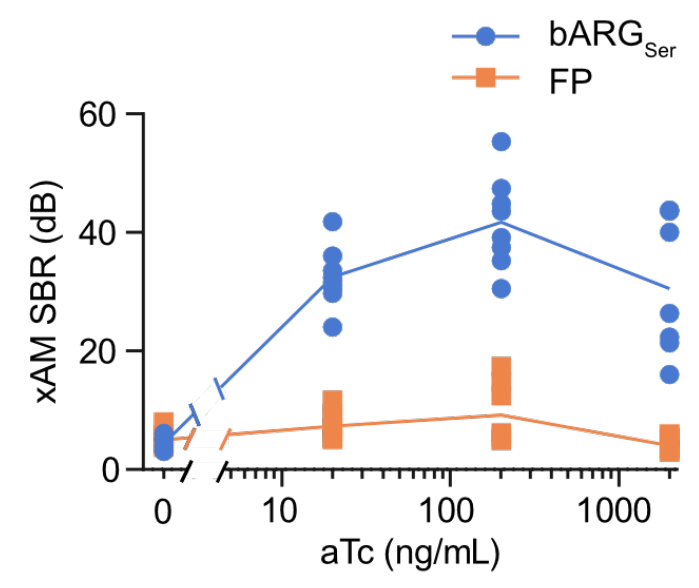

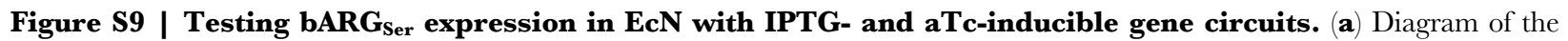
IPTG-inducible construct used to express bARGser in $\mathrm{EcN}$ (top), and representative optical and xAM images of bARGser-expressing or fluorescent protein (FP)-expressing patches of $\mathrm{EcN}$ on solid media with varying IPTG concentrations at $37^{\circ} \mathrm{C}$ (bottom). (b) Quantification of the xAM SBR of all patches from the experiment in (a) (n=8). (c-d) Same as in (a-b) but for the aTc-inducible construct. The scale bars in $(\mathrm{a}, \mathrm{c})$ represent $1 \mathrm{~cm}$. 
a
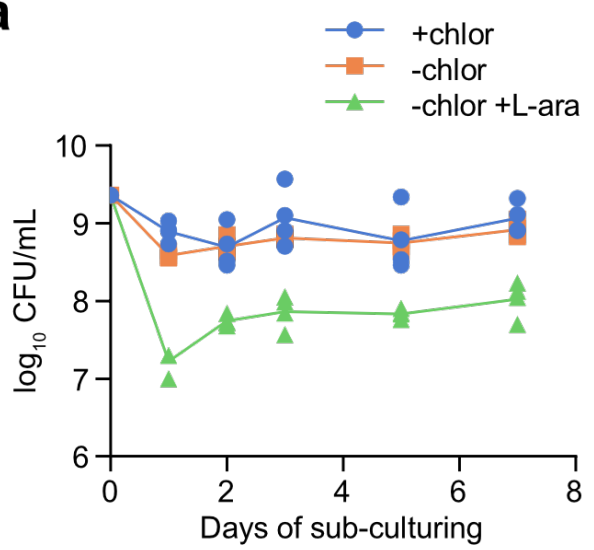

b

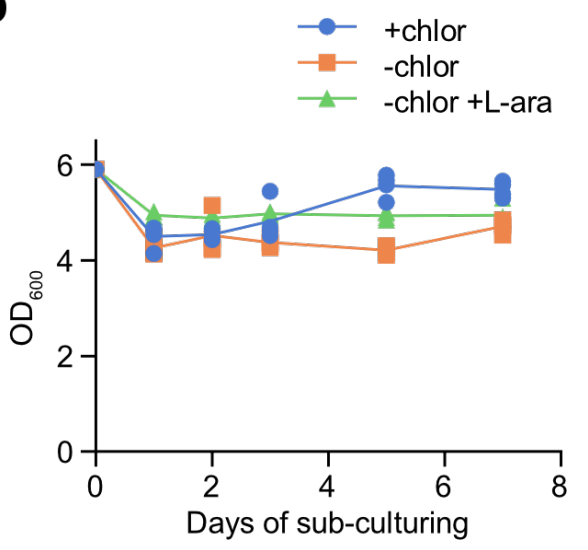

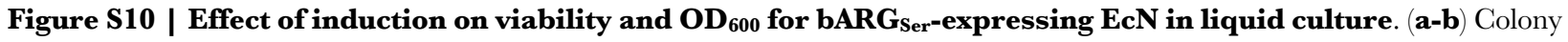
forming units (CFU) per $\mathrm{mL}$ of culture (a) and optical density at $600 \mathrm{~nm}$ (b) during daily sub-culturing into LB media with 25 $\mu \mathrm{g} / \mathrm{mL}$ chloramphenicol (+chlor), without chloramphenicol (-chlor), or without chloramphenicol and with $0.1 \%$ (w/v) L-arabinose (-chlor +L-ara) using pBAD-bARGser-AxeTxe EcN. 
bioRxiv preprint doi: https://doi.org/10.1101/2021.04.26.441537; this version posted October 29, 2021. The copyright holder for this preprint (which was not certified by peer review) is the author/funder. All rights reserved. No reuse allowed without permission.

a

\begin{tabular}{|c|c|c|c|c|c|c|}
\hline \multirow[b]{2}{*}{ Condition } & \multirow[b]{2}{*}{ Replicate } & \multicolumn{5}{|c|}{ Non-white mutants / total colonies screened } \\
\hline & & Starter & Day 1 & Day 2 & Day 3 & Day 5 \\
\hline +chlor & \#1 & $0 / 226$ & $0 / 68$ & $0 / 69$ & $0 / 60$ & $0 / 40$ \\
\hline +chlor & \#2 & & $0 / 55$ & $0 / 47$ & $0 / 355$ & $0 / 54$ \\
\hline +chlor & \#3 & & $0 / 48$ & $0 / 39$ & $0 / 98$ & $0 / 42$ \\
\hline +chlor & \#4 & & $0 / 56$ & $0 / 21$ & $0 / 49$ & $0 / 233$ \\
\hline -chlor & \#1 & & $0 / 39$ & $0 / 54$ & $0 / 70$ & $0 / 64$ \\
\hline -chlor & \#2 & & $0 / 28$ & $0 / 46$ & $0 / 65$ & $0 / 46$ \\
\hline -chlor & \#3 & & $0 / 47$ & $0 / 57$ & $0 / 75$ & $0 / 52$ \\
\hline -chlor & \#4 & & $0 / 36$ & $0 / 57$ & $0 / 57$ & $0 / 38$ \\
\hline -chlor +ara & \#1 & & $0 / 2$ & $0 / 68$ & $0 / 53$ & $0 / 44$ \\
\hline -chlor +ara & \#2 & & $0 / 2$ & $0 / 62$ & $2 / 86$ & $0 / 68$ \\
\hline -chlor +ara & \#3 & & ND* & $0 / 67$ & $3 / 102$ & $6 / 70$ \\
\hline -chlor +ara & \#4 & & $0 / 1$ & $0 / 76$ & $0 / 34$ & $0 / 56$ \\
\hline
\end{tabular}

*ND: no data because there were no colonies

b

3-D8
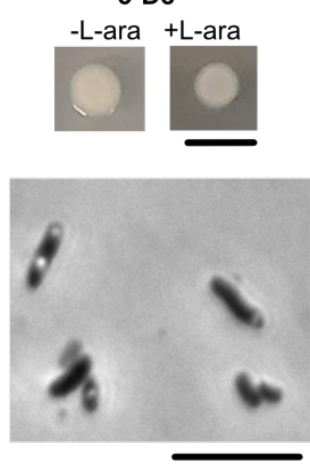

3-H3
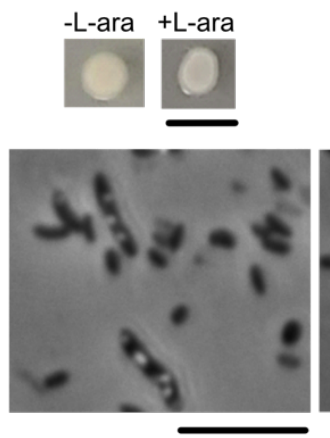

3-H7
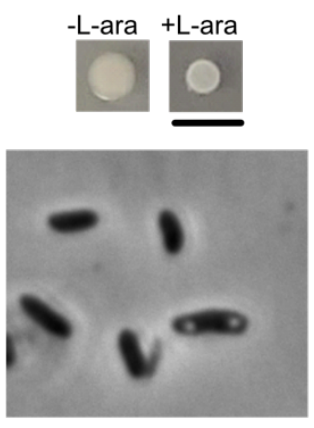

3-E1
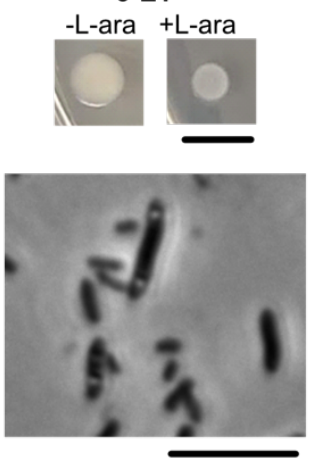

3-H4
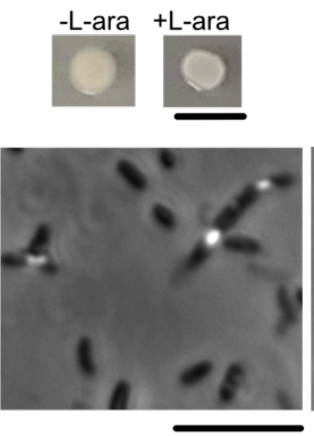

3-H8

-L-ara +L-ara
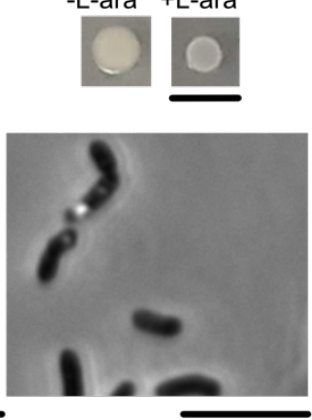

3-E3
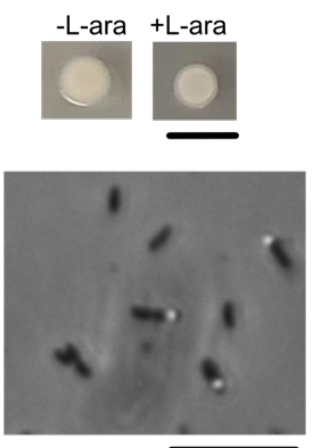

3-H5
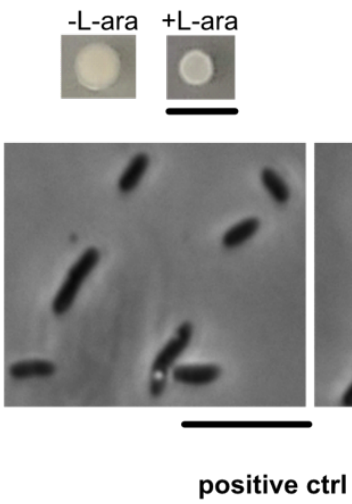

-L-ara +L-ara
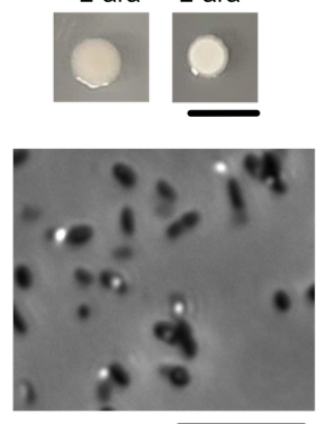

3-E5
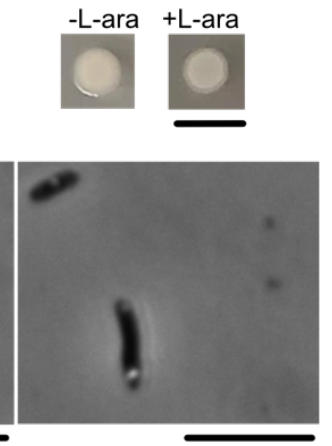

3-H6
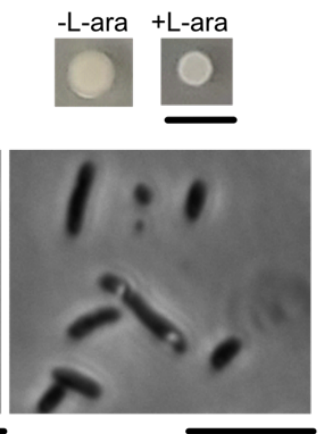

negative ctrl

-L-ara +L-ara
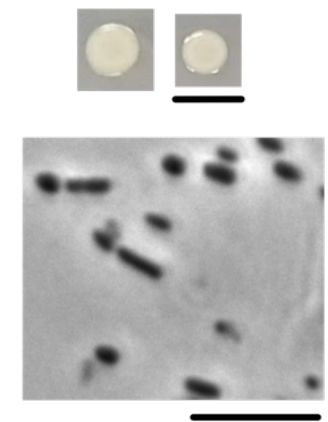
bioRxiv preprint doi: https://doi.org/10.1101/2021.04.26.441537; this version posted October 29, 2021. The copyright holder for this preprint (which was not certified by peer review) is the author/funder. All rights reserved. No reuse allowed without permission.

Figure S11 | Quantification and characterization of EcN mutants deficient in bARG daily subculturing in vitro. (a) Numbers of non-white mutant colonies and total colonies screened on plates with $0.1 \%(\mathrm{w} / \mathrm{v})$ L-arabinose from daily sub-culturing into LB media with $25 \mu \mathrm{g} / \mathrm{mL}$ chloramphenicol (+chlor), without chloramphenicol (-chlor), or without chloramphenicol and with $0.1 \%(\mathrm{w} / \mathrm{v})$ L-arabinose (-chlor +L-ara) using pBAD-bARGser-AxeTxe EcN. Cultures where mutants were found are indicated in red. (b) Optical images of patches (top rows) on fresh plates with $0.1 \%(\mathrm{w} / \mathrm{v}) \mathrm{L}-\mathrm{arabinose}(+\mathrm{L}-$ ara) and without L-arabinose (-L-ara), and phase contrast microscopy images (bottom rows) from the 11 mutant colonies in (a). Mutants 3-D3 and 3-E1 were from the culture -chlor + ara, replicate \#2, day 3; mutants 3-E3, 3-E4, and 3-E5 were from the culture -chlor +ara, replicate \#3, day 3; and mutants 3-H3 through 3-H8 were from the culture -chlor +ara, replicate \#3, day 5. The positive and negative controls were wild-type pBAD-bARGser-AxeTxe EcN and pBAD-FP-AxeTxe EcN, respectively. Scale bars are $1 \mathrm{~cm}$ for images of patches and $10 \mu \mathrm{m}$ for microscopy images. 
bioRxiv preprint doi: https://doi.org/10.1101/2021.04.26.441537; this version posted October 29, 2021. The copyright holder for this preprint (which was not certified by peer review) is the author/funder. All rights reserved. No reuse allowed without permission.

a

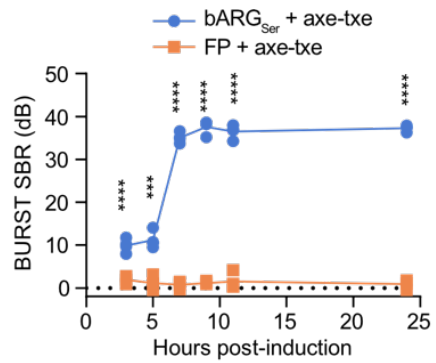

b

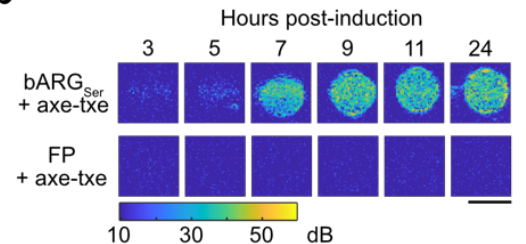

C

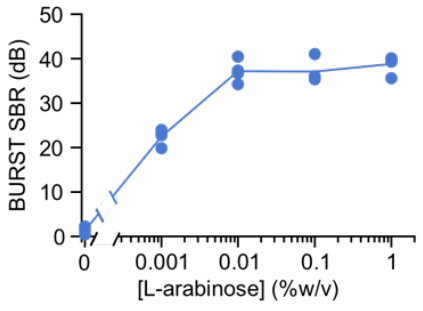

e

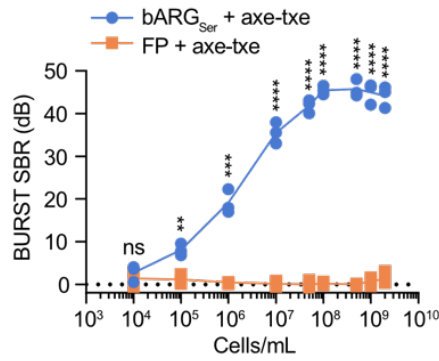

d

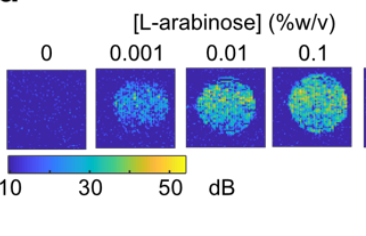

f

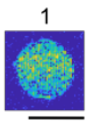

Cells $/ \mathrm{mL}$

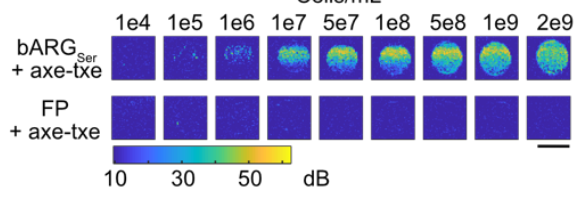

Figure S12 | In vitro characterizations of bARGser-expressing EcN with BURST ultrasound imaging. (a-b) BURST ultrasound signal-to-background ratio (SBR) versus time after inducing pBAD-bARGser-AxeTxe and pBAD-FP-AxeTxe EcN strains with $0.1 \%$ L-arabinose in liquid culture at $37^{\circ} \mathrm{C}$ (a) and the corresponding representative BURST images (b). (c-d) BURST ultrasound SBR versus L-arabinose concentration used to induce pBAD-bARGser-AxeTxe EcN in liquid culture at $37^{\circ} \mathrm{C}$ for 24 hours (c) and the corresponding representative BURST images. (e-f) BURST ultrasound SBR versus concentration of pBAD-

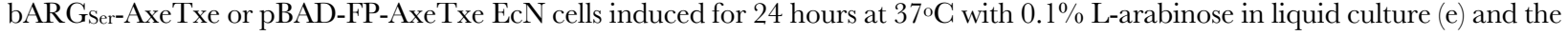
corresponding representative BURST images (f). Note that the BURST SBR saturated at 7 hours post-induction, $0.01 \%$ (w/v) Larabinose, and $10^{8}$ cells $/ \mathrm{mL}$. All scale bars are $2 \mathrm{~mm}$. For (a-d), cells were normalized to $10^{9}$ cells $/ \mathrm{mL}$ in agarose phantoms for ultrasound imaging. For (a, c, e), each point is a biological replicate $(\mathrm{n}=4$ for a and $\mathrm{c} ; \mathrm{n}=3$ for e) that is the average of at least 2 technical replicates. Asterisks represent statistical significance by unpaired t tests $(* * * *=p<0.0001, * * *=p<0.001, * *=p<0.01, n s$ $=$ no significance). 
a
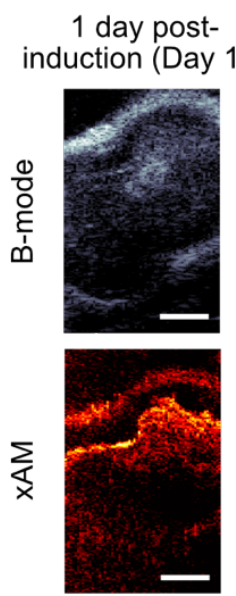

b Re-expressed
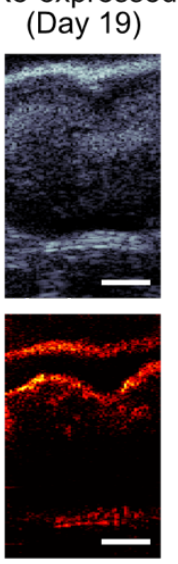

C Uninduced
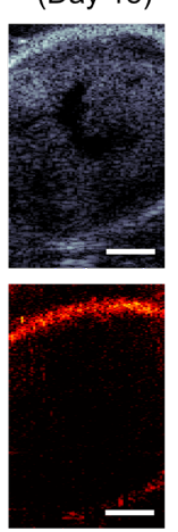

d FP (control)
(Day 18)
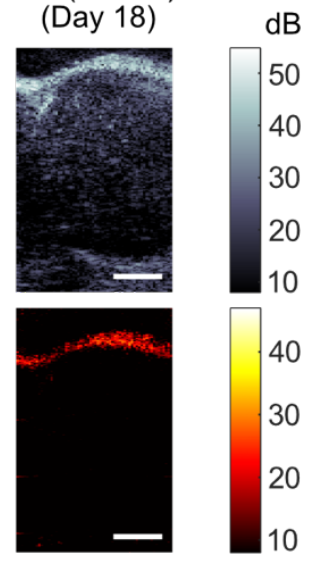

e

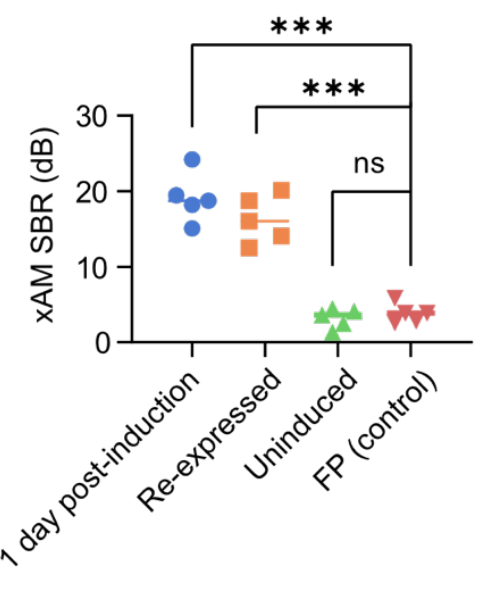

Figure S13 | xAM ultrasound imaging of mouse tumors colonized by EcN. (a-c) Representative B-mode (top, grayscale) and xAM (bottom, hot-scale) ultrasound images of tumors colonized by pBAD-bARGser-AxeTxe EcN at least 24 hours after induction with L-arabinose on day 18 (a), at least 24 hours after collapse and re-induction (day 19) (b), or uninduced on day 18 (c). (d) Representative ultrasound images of tumors colonized by pBAD-FP-AxeTxe EcN at least 24 hours after induction with Larabinose on day 18. Scale bars in (a-d) represent $2 \mathrm{~mm}$. (e) Quantification of the xAM SBR for the same conditions in (a-d). Each group is $\mathrm{N}=5$. Asterisks represent statistical significance by unpaired t tests $(* * *=p<0.001$, ns $=$ no significance). See Fig. 4a for the corresponding in vivo protocol. 
bioRxiv preprint doi: https://doi.org/10.1101/2021.04.26.441537; this version posted October 29, 2021. The copyright holder for this preprint (which was not certified by peer review) is the author/funder. All rights reserved. No reuse allowed without permission.

DAPI

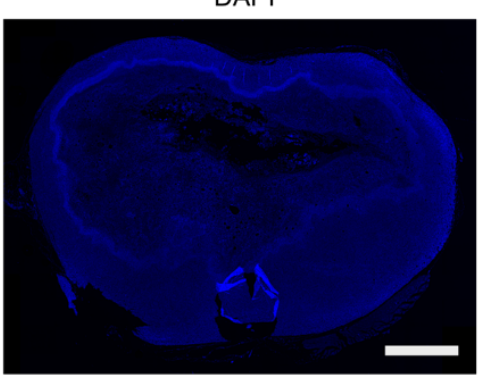

Rabbit $\operatorname{lgG}$ (control)

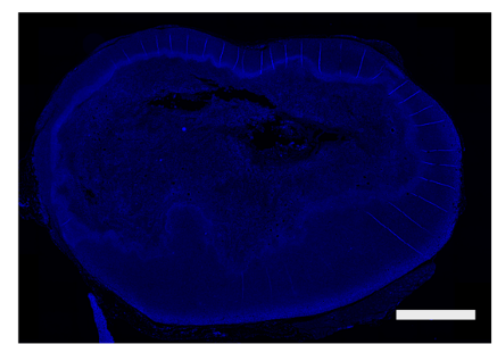

Opal 520
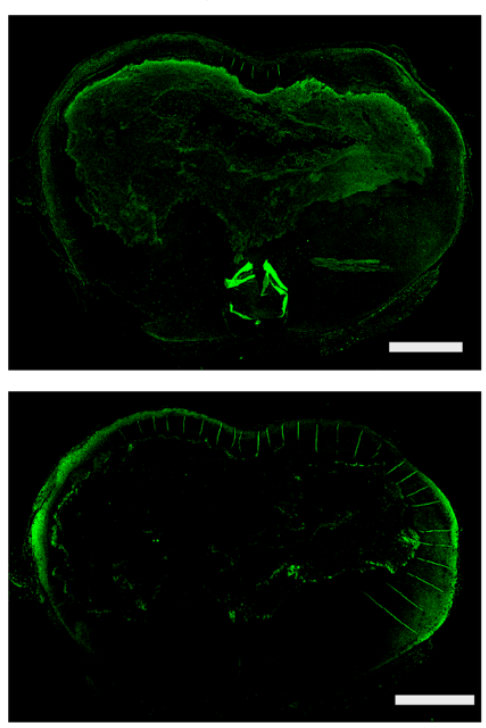

Overlay
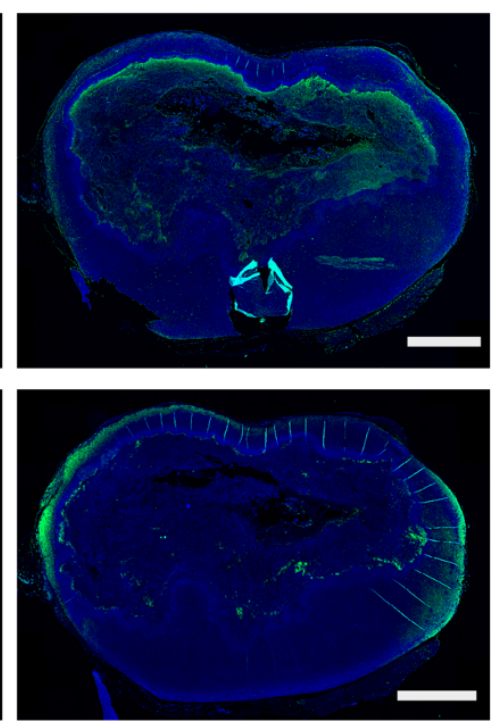

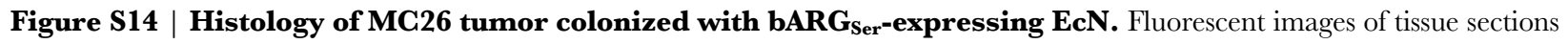
after ultrasound imaging on day 19 (see Fig. 4a). Sections were incubated with either polyclonal rabbit anti-E. coli antibodies (top row) or non-reactive rabbit IgG isotype control antibody (bottom row) as a negative control. All sections were then incubated with an Opal 520 polymer anti-rabbit HRP antibody (Akoya biosciences) and counterstained with DAPI. The EcN are visible in the necrotic core in the Opal 520 channel (top middle panel); the edges of the tissue exhibit a high degree of background staining (bottom middle panel). 
bioRxiv preprint doi: https://doi.org/10.1101/2021.04.26.441537; this version posted October 29, 2021. The copyright holder for this preprint (which was not certified by peer review) is the author/funder. All rights reserved. No reuse allowed without permission.

a

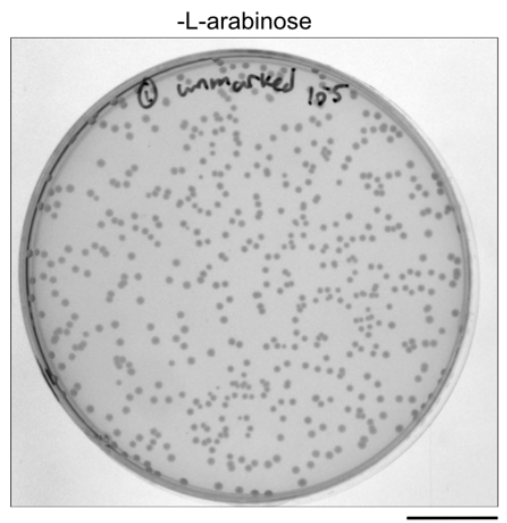

b

\begin{tabular}{ccc}
\hline Mouse & Condition & $\begin{array}{c}\text { Non-white mutants / total } \\
\text { colonies screened }\end{array}$ \\
\hline$\# 1$ & Induced & $0 / 687$ \\
$\# 2$ & Induced & $2 / 807$ \\
$\# 3$ & Induced & $0 / 341$ \\
$\# 4$ & Induced & $0 / 478$ \\
$\# 5$ & Induced & $5 / 585$ \\
$\# 6$ & Uninduced & $0 / 660$ \\
$\# 7$ & Uninduced & $0 / 567$ \\
$\# 8$ & Uninduced & $0 / 553$ \\
$\# 9$ & Uninduced & $0 / 556$ \\
$\# 10$ & Uninduced & $0 / 863$ \\
\hline Total & & $7 / 6097 \sim 0.1 \%$ \\
\hline
\end{tabular}

C

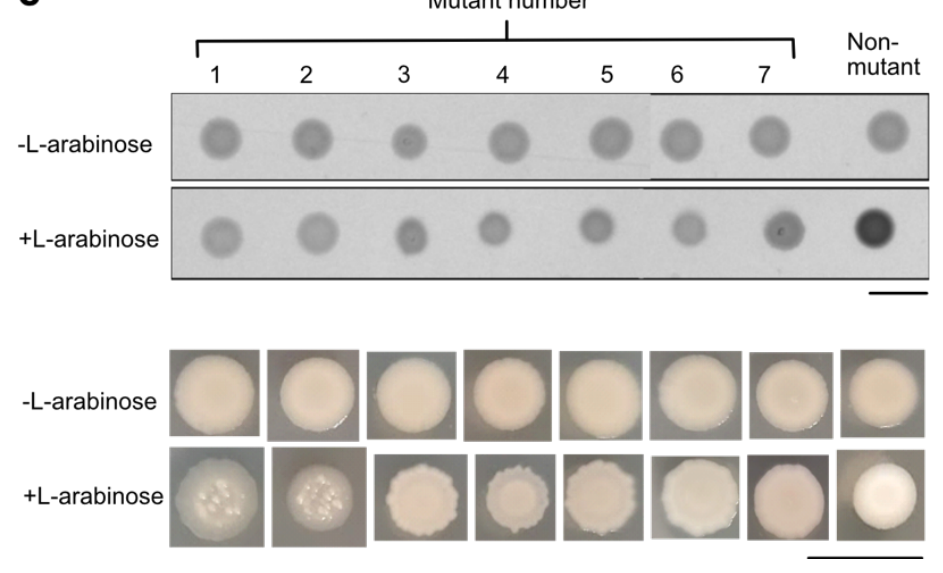

Figure S15 | Screening for EcN mutants defective in bARG White light transmission images of plates with $0.1 \%(\mathrm{w} / \mathrm{v})$ L-arabinose and without L-arabinose from plating a tumor (from mouse \#5 in (b)) colonized by bARG Ser-expressing EcN. Mutant colonies on the +L-arabinose plate appear lighter (more translucent) than wild-type opaque colonies and are indicated by red circles. (b) Numbers of non-white mutant colonies and total colonies screened on plates with $0.1 \%(\mathrm{w} / \mathrm{v})$ L-arabinose for the ten mice injected with pBAD-bARGser-AxeTxe EcN. c, White light transmission images (top) and photographs (bottom) of patches on fresh plates with $0.1 \%(\mathrm{w} / \mathrm{v})$ L-arabinose and without L-arabinose made from the seven translucent mutant colonies in red in (b) and an opaque non-mutant colony as a control. Mutants 1-2 were from mouse \#2 and mutants 3-7 were from mouse \#5. Scale bars are $2 \mathrm{~cm}$ in (a) and $1 \mathrm{~cm}$ in (c). 
bioRxiv preprint doi: https://doi.org/10.1101/2021.04.26.441537; this version posted October 29, 2021. The copyright holder for this preprint (which was not certified by peer review) is the author/funder. All rights reserved. No reuse allowed without permission.

a

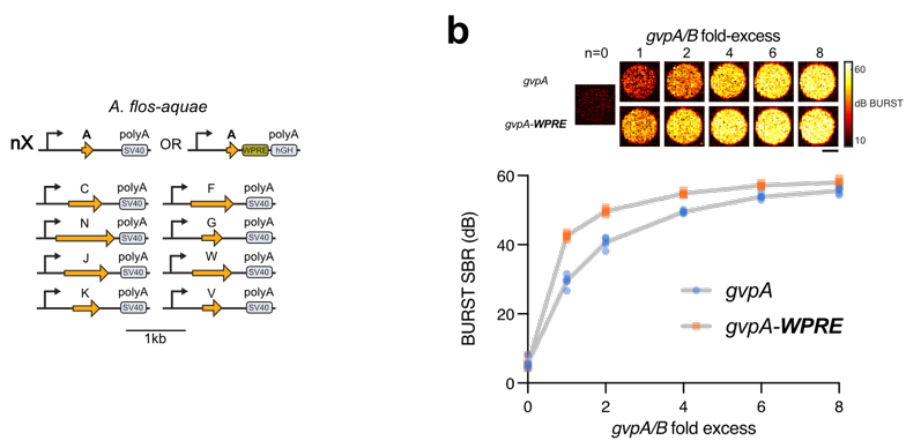

C

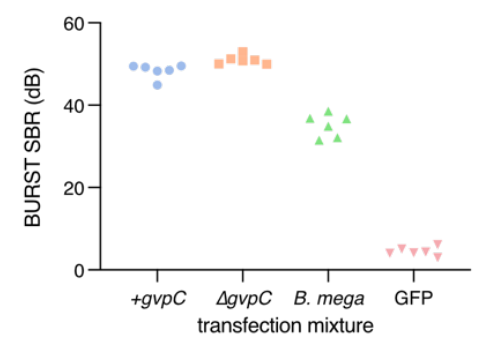

d
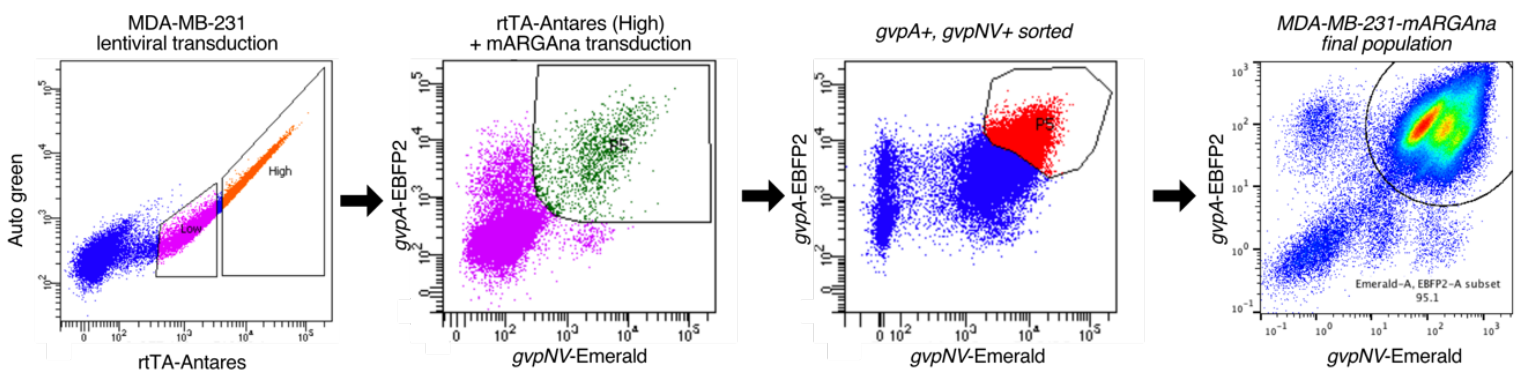

e
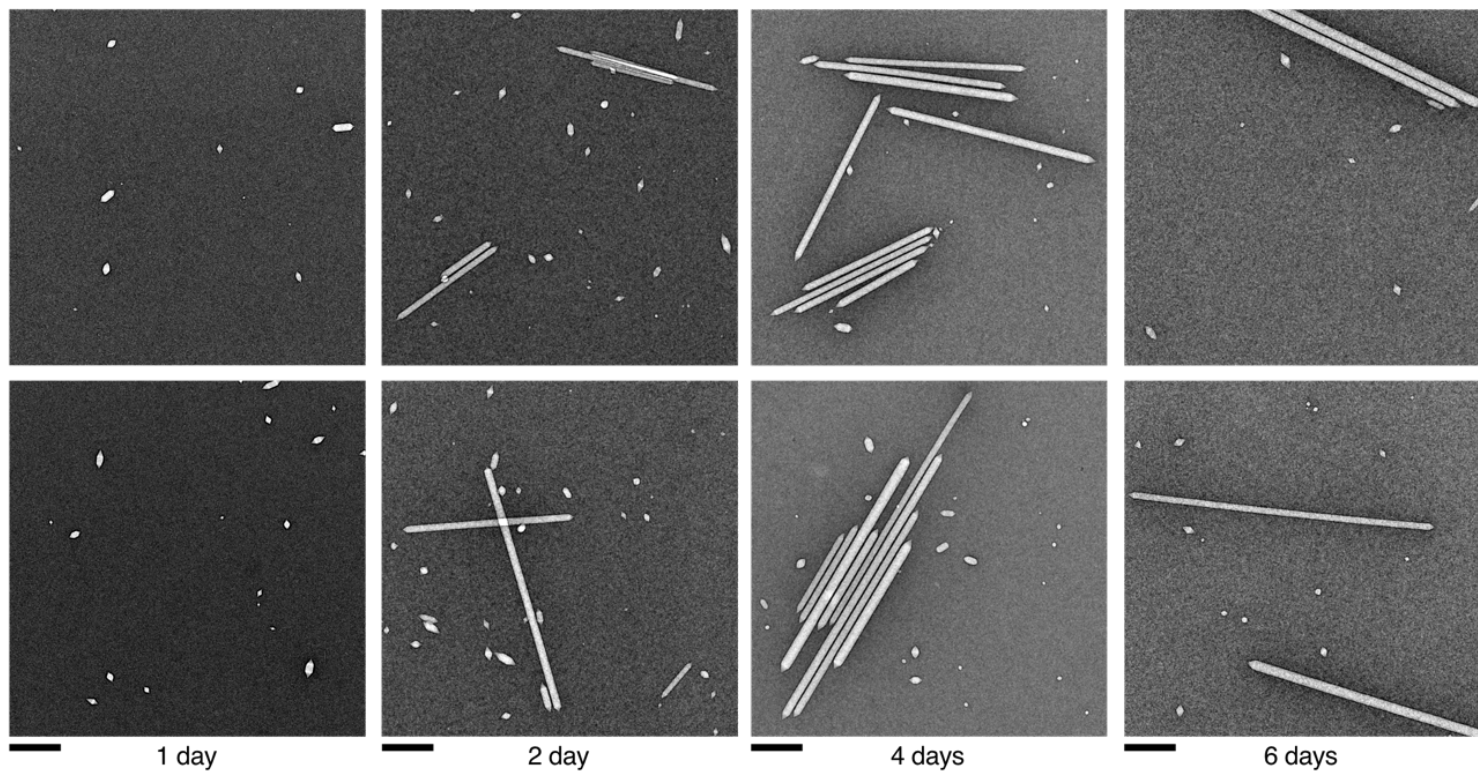

4 days

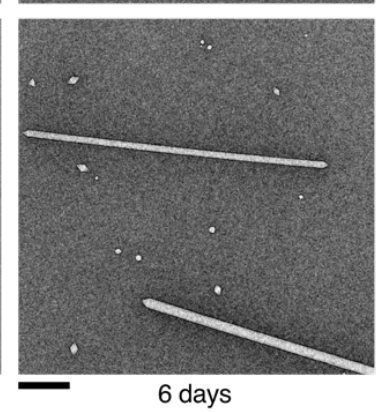

Figure S16 | Additional data on heterologous expression of the A. flos-aquae GV gene cluster in mammalian cells. (a) Schematic of the codon-optimized A. flos-aquae monocistronic plasmid set used in this study. (b) Representative BURST images (top) and SBR quantification ( $\mathrm{n}=5$, bottom) of transient GV expression in HEK293T cells 3 days after cotransfection of mixtures with varying gopA fold excess relative to their respective assembly factor plasmids, with and without WPRE elements on the gopA DNA. Gray lines connect the means of replicates. (c) BURST SBR quantification $(\mathrm{n}=6)$ of 293 T cells expressing constructs

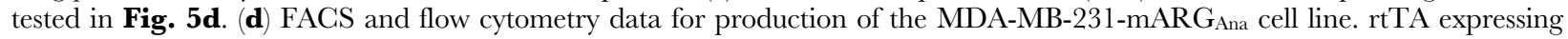
"High" cells were collected for subsequent mARG/Transposase transduction. Cells expressing gvpA and gvpNV were sorted twice to arrive at the final $\sim 95 \%$ pure population. (e) TEM images of GVs purified from MDA-MB-231-mARGAna detergent lysates after $1,2,4$ and 6 days of $1 \mu \mathrm{g} / \mathrm{ml}$ doxycycline induction. Scalebars represent $0.5 \mu \mathrm{m}$. 
bioRxiv preprint doi: https://doi.org/10.1101/2021.04.26.441537; this version posted October 29, 2021. The copyright holder for this preprint (which was not certified by peer review) is the author/funder. All rights reserved. No reuse allowed without permission.

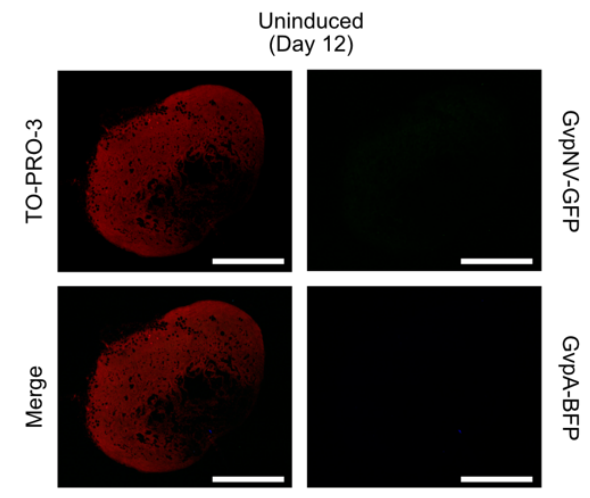

Figure S17 | Uninduced tumor micrograph. Fluorescence micrograph of a $100 \mathrm{~nm}$ thick tissue section from an uninduced tumor. The red color shows TO-PRO-3 nuclear stain; GFP and BFP fluorescence were not observed for this control tumor. Scale bars represent $2 \mathrm{~mm}$. 